\title{
ANOMALOUS DIFFUSION IN COMB-SHAPED DOMAINS AND GRAPHS.
}

\author{
SAMUEL COHN $^{1}$, GAUTAM IYER $^{1}$, JAMES NOLEN $^{2}$, AND ROBERT L. PEGO ${ }^{1}$
}

\begin{abstract}
In this paper we study the asymptotic behavior of Brownian motion in both comb-shaped planar domains, and comb-shaped graphs. We show convergence to a limiting process when both the spacing between the teeth and the width of the teeth vanish at the same rate. The limiting process exhibits an anomalous diffusive behavior and can be described as a Brownian motion time-changed by the local time of an independent sticky Brownian motion. In the two dimensional setting the main technical step is an oscillation estimate for a Neumann problem, which we prove here using a probabilistic argument. In the one dimensional setting we provide both a direct SDE proof, and a proof using the trapped Brownian motion framework in Ben Arous et al. (Ann. Probab. '15).
\end{abstract}

\section{Introduction.}

Diffusion in comb-like structures arises in the study of several applications such as the study of linear porous media, microscopically disordered fluids, transport in dendrites and tissues (see for instance [You88, ADH90, SW91, BE07, DBMZ07] and references therein). Our aim in this paper is to study idealized, periodic, comb-shaped domains in $\mathbb{R}^{2}$ under scaling regimes where an anomalous diffusive behavior is observed. We also study scaling limits of a skew Brownian motion on an infinite comb-shaped graph. In both scenarios we show that under a certain scaling the limiting process is a Brownian motion time-changed by the local time of an independent sticky reflected Brownian motion. We describe each of these scenarios separately in Sections 1.1 and 1.2 below.

1.1. Anomalous Diffusion in Comb-Shaped Domains. Let $h_{0} \in(0, \infty]$, and $\alpha, \varepsilon>0$, and let $\Omega_{\varepsilon} \subset \mathbb{R}^{2}$ be the fattened comb-shaped domain defined by

$$
\Omega_{\varepsilon}=\left\{(x, y) \in \mathbb{R}^{2} \mid-\varepsilon<y<h_{0} \mathbf{1}_{B\left(\varepsilon \mathbb{Z}, \alpha \varepsilon^{2} / 2\right)}(x)\right\},
$$

where $B\left(\varepsilon \mathbb{Z}, \alpha \varepsilon^{2} / 2\right) \subseteq \mathbb{R}$ denotes the $\alpha \varepsilon^{2} / 2$ neighborhood of $\varepsilon \mathbb{Z}$, and $\mathbf{1}$ denotes the indicator function. Figure 1 shows a picture of the domain $\Omega_{\varepsilon}$. We refer to the

\footnotetext{
1 Department of Mathematical Sciences, Carnegie Mellon University, Pittsburgh, PA 15213.

2 Duke University, Department of Mathematics, 243 Physics Bullding, Durham, NC 27708 .

E-mail addresses: samuelcohn032@gmail.com, gautam@math.cmu.edu, nolen@math.duke.edu, rpego@cmu.edu.

2010 Mathematics Subject Classification. Primary 60G22; Secondary 35B27.

This material is based upon work partially supported by the National Science Foundation under grants DMS-1252912, DMS-1351653, DMS-1515400, DMS-1814147, and the Center for Nonlinear Analysis.
} 
region where $-\varepsilon<y<0$ as the spine; $\Omega_{\varepsilon}$ also has teeth of height $h_{0}$ and width $\alpha \varepsilon^{2}$, which are spaced $\varepsilon$ apart.

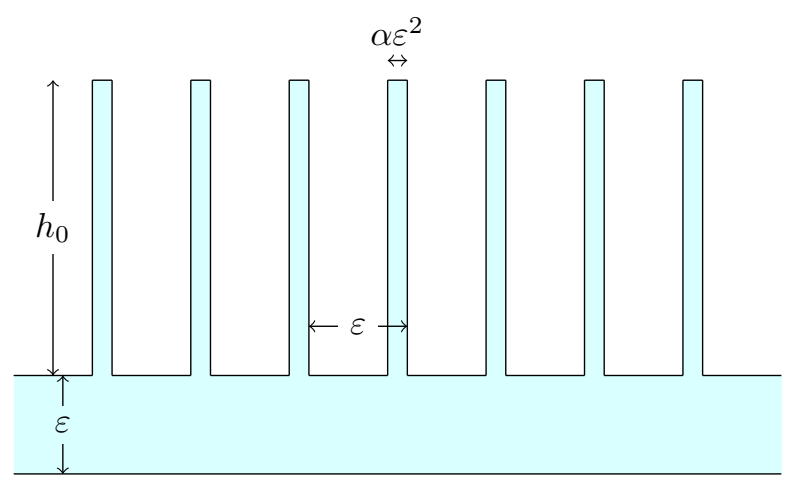

Figure 1. Image of the comb-shaped domain $\Omega_{\varepsilon}$. The teeth have width $\alpha \varepsilon^{2}$ and height $h_{0}$. The spine has width $\varepsilon$, and the teeth are spaced a distance of $\varepsilon$ apart.

Let $Z^{\varepsilon}=\left(X^{\varepsilon}, Y^{\varepsilon}\right)$ be a Brownian motion in $\Omega_{\varepsilon}$ that is reflected normally on the boundary $\partial \Omega_{\varepsilon}$. Our aim is to study the limiting behavior of $Z^{\varepsilon}$ as $\varepsilon \rightarrow 0$. This is an idealized, two dimensional, version of the arterial flow models considered by Young [You88]. Note that the process $Z^{\varepsilon}$ may travel large horizontal distances when it is in the spine, but travels only negligible horizontal distances when it is "trapped" inside the teeth. From the shape of $\Omega_{\varepsilon}$, one expects that the chance $Z^{\varepsilon}$ wanders into the teeth from the spine is of order $\alpha \varepsilon$. Since the teeth are spaced $\varepsilon$ apart, the process $Z^{\varepsilon}$ encounters $O(1 / \varepsilon)$ teeth after traveling an $O(1)$ distance horizontally. These balance, and after large horizontal distances, the process $Z^{\varepsilon}$ spends comparable amounts of time in the spine and in the teeth. This leads us to expect that the limiting horizontal behavior of $Z^{\varepsilon}$ should be described by a Brownian motion that is time-changed so that it only moves when the process is in the spine - this is our main result.

To state the result, we let $\Omega_{0} \stackrel{\text { def }}{=} \mathbb{R} \times\left[0, h_{0}\right]$, and let $\pi_{\varepsilon}: \Omega_{\varepsilon} \rightarrow \Omega_{0}$ be defined by $\pi_{\varepsilon}(x, y)=\left(x, y^{+}\right)$, where $y^{+}=\max \{y, 0\}$ denotes the positive part of $y$. Given a probability measure $\mu^{\varepsilon}$ on $\Omega_{\varepsilon}$, let $\pi_{\varepsilon}^{*}\left(\mu^{\varepsilon}\right)$ denote the push forward of $\mu^{\varepsilon}$, under the map $\pi_{\varepsilon}$, to a probability measure on $\Omega_{0}$. We can now state the main result.

Theorem 1.1. Let $Z^{\varepsilon}=\left(X^{\varepsilon}, Y^{\varepsilon}\right)$ be a normally reflected Brownian motion in $\Omega_{\varepsilon}$ with initial distribution $\mu^{\varepsilon}$. If the sequence of measures $\left(\pi_{\varepsilon}^{*}\left(\mu^{\varepsilon}\right)\right)$ converges weakly to a probability measure $\mu$ on $\Omega_{0}$, then the sequence of processes $Z^{\varepsilon,+} \stackrel{\text { def }}{=} \pi_{\varepsilon}\left(Z^{\varepsilon}\right)$ converges weakly as $\varepsilon \rightarrow 0$. The limiting process, denoted by $Z=(X, Y)$, can be described as follows. The initial distribution of $Z$ is $\mu$. The process $Y$ is a Brownian motion on $\left(0, h_{0}\right)$, which is normally reflected at $h_{0}$ if $h_{0}<\infty$, and is stickily reflected (with parameter $1 / \alpha$ ) at 0 . The process $X$ is a time-changed Brownian motion given by

$$
X_{t}=\bar{W}_{\frac{2}{\alpha}} L_{t}^{Y}(0)
$$

where $\bar{W}$ is a Brownian motion on $\mathbb{R}$ that is independent of $Y$, and $L^{Y}(0)$ is the local time of $Y$ at 0 . 
To clarify notation, we follow the normalization convention of [KS91], and define local time of $Y$ at 0 by

$$
L_{t}^{Y}(0)=\lim _{\delta \rightarrow 0} \frac{1}{2 \delta} \int_{0}^{t} \mathbf{1}_{\left\{0 \leqslant Y_{s} \leqslant \delta\right\}} d\langle Y\rangle_{s}=\lim _{\delta \rightarrow 0} \frac{1}{2 \delta} \int_{0}^{t} \mathbf{1}_{\left\{0<Y_{s} \leqslant \delta\right\}} d s .
$$

In the second equality above we note that the strict inequality $0<Y_{s}$ in the integrand is crucial, as the process $Y$ spends a non-negligible time at 0 . Indeed, recall that the sticky reflection of the process $Y$ at 0 is characterized by the local time relation

$$
2 d L_{t}^{Y}(0)=\alpha \mathbf{1}_{\left\{Y_{t}=0\right\}} d t .
$$

Such a process can be constructed explicitly by time changing a reflected Brownian motion, or by using the Hille-Yosida theorem. We elaborate on this in Section 2, below.

We remark that while the statement of Theorem 1.1 is intuitive, the proof isn't as simple. The broad outline of the proof follows techniques introduced by Freidlin and Wentzell (see for instance Theorem 8.2.2 in [FW12]) and the structure in [HKPG16, $\left.\mathrm{HIK}^{+} 18\right]$. However, the key step in establishing the required estimates requires balancing the time spent by $Z^{\varepsilon}$ in the spine with the local time at the interface between the teeth and spine. In order to prove this, we require an oscillation estimate on the solution to a certain Neumann problem (Proposition 3.8, below).

To the best of our knowledge, the oscillation estimate we require can not be obtained by standard techniques for the following reasons: First, for the problem at hand energy methods only provide estimates with domain dependent constants. Since $\Omega_{\varepsilon}$ varies with $\varepsilon$ these constants may degenerate as $\varepsilon \rightarrow 0$. Second, since we impose Neumann boundary conditions on the entire boundary we may not easily use techniques based on the comparison principle. We prove the oscillation estimate here directly by using a probabilistic argument, and this comprises the bulk of the proof of Theorem 1.1.

Notice that Theorem 1.1 immediately yields the behavior of the variance of the horizontal displacement. This question has been studied by various authors (see for instance [BDB14] and references therein), and is of interest as it is an easily computable benchmark indicating anomalous diffusion.

Corollary 1.2. If $h_{0}<\infty$ then

$$
\begin{gathered}
\lim _{t \rightarrow 0} \lim _{\varepsilon \rightarrow 0} \frac{1}{t} \boldsymbol{E}^{(x, 0)}\left|X_{t}^{\varepsilon}-x\right|^{2}=1, \\
\lim _{t \rightarrow \infty} \lim _{\varepsilon \rightarrow 0} \frac{1}{t} \boldsymbol{E}^{(x, 0)}\left|X_{t}^{\varepsilon}-x\right|^{2}=\frac{1}{\alpha h_{0}+1} .
\end{gathered}
$$

If $h_{0}=\infty$, then (1.3a) still holds. However, instead of (1.3b) we have

$$
\lim _{t \rightarrow \infty} \lim _{\varepsilon \rightarrow 0} \frac{1}{\sqrt{t}} \boldsymbol{E}^{(x, 0)}\left|X_{t}^{\varepsilon}-x\right|^{2}=\frac{1}{\alpha}\left(\frac{8}{\pi}\right)^{1 / 2} .
$$

Here we clarify that the notation $\boldsymbol{E}^{(x, 0)}$ refers to the expectation under the probability measure $\boldsymbol{P}^{(x, 0)}$ under which $\left(X_{0}^{\varepsilon}, Y_{0}^{\varepsilon}\right)=(x, 0)$ almost surely. Note that when $h_{0}<\infty$, the variance is asymptotically linear with slope 1 at short time, and asymptotically linear at long time with slope strictly smaller than 1 . On the other hand, when $h_{0}=\infty$ the variance is asymptotically linear for short time, and 
asymptotically $O(\sqrt{t})$ for long time, indicating an anomalous sub-diffusive behavior on long time scales. This was also previously observed by Young [You88].

In addition to the variance, another quantity of interest is the limiting behavior of the probability density function. This is essentially a PDE homogenization result that also follows quickly from Theorem 1.1. Explicitly, let $u^{\varepsilon}$ represent the concentration density of a scalar diffusing in the region $\Omega_{\varepsilon}$. When the diffusivity is normalized to be $1 / 2$, and the boundaries are impermeable the time evolution of $u^{\varepsilon}$ is governed by the heat equation with Neumann boundary conditions:

$$
\begin{array}{cc}
\partial_{t} u^{\varepsilon}-\frac{1}{2} \Delta u^{\varepsilon}=0 & \text { in } \Omega_{\varepsilon} \\
\partial_{\nu} u^{\varepsilon}=0 & \text { on } \partial \Omega_{\varepsilon} .
\end{array}
$$

Using Theorem 1.1 we can show that $u^{\varepsilon}$ converges as $\varepsilon \rightarrow 0$, and obtain effective equations for the limit. The same equations were also obtained heuristically by Young [You88].

Corollary 1.3. Let $u_{0}: \Omega_{0} \rightarrow \mathbb{R}$ be a bounded continuous function, and let $u^{\varepsilon}$ be the solution to (1.5a)-(1.5b) with initial data $u_{0} \circ \pi_{\varepsilon}$. Let $\mu^{\varepsilon}$ be a family of test probability measures on $\Omega_{\varepsilon}$ such that $\left(\pi_{\varepsilon}^{*}\left(\mu^{\varepsilon}\right)\right)$ converges weakly to a probability measure $\mu$ on $\Omega_{0}$. Then for any $t>0$ we have

$$
\lim _{\varepsilon \rightarrow 0} \int_{\Omega_{\varepsilon}} u^{\varepsilon}(z, t) d \mu^{\varepsilon}(z)=\int_{\Omega_{0}} u(z, t) d \mu(z),
$$

where $u: \Omega_{0} \rightarrow \mathbb{R}$ is the unique solution of the system

$$
\begin{array}{cl}
\partial_{t} u-\frac{1}{2} \partial_{y}^{2} u=0, & \text { for } t>0, y \in\left(0, h_{0}\right), \\
\alpha \partial_{y} u+\partial_{x}^{2} u=\partial_{y}^{2} u, & \text { when } y=0, \\
\partial_{y} u=0 & \text { when } y=h_{0}, \\
u=u_{0} & \text { when } t=0 .
\end{array}
$$

Since large scale transport only occurs in the $x$-direction, one is often only interested in the limiting behavior in this direction. This can be obtained by taking the slice of $u$ at $y=0$, leading to a self contained time fractional equation, similar to the Basset equation [Bas87]. We remark that such time fractional PDEs associated with the time-changed diffusions have been studied in more generality in [BMN09] (see also [Coh18, MS15]), and we refer the reader to these papers for the details.

Proposition 1.4. Let $v(x, t)=u(x, 0, t)$, where $u$ is the solution of $(1.7 \mathrm{a})-(1.7 \mathrm{~d})$. Then $v$ satisfies

$$
\partial_{t} v+\frac{\alpha}{2} \partial_{t}^{w} v-\frac{1}{2} \partial_{x}^{2} v=\frac{\alpha}{2} f
$$

with initial data $v(x, 0)=u_{0}(x, 0)$. The operator $\partial_{t}^{w}$ appearing above is a generalized Caputo derivative defined by

$$
\partial_{t}^{w} v(x, t) \stackrel{\text { def }}{=} \int_{0}^{t} w(t-s) \partial_{t} v(x, s) d s
$$

where $w$ is defined by

$$
w(t) \stackrel{\text { def }}{=} \frac{2}{h_{0}} \sum_{k=0}^{\infty} \exp \left(-\frac{(2 k+1)^{2} \pi^{2} t}{8 h_{0}^{2}}\right) .
$$


The function $f$ appearing on the right of (1.8) can be explicitly determined in terms of $u_{0}$ by the identity $f=f(x, t)=\partial_{y} g(x, 0, t)$, where $g=g(x, y, t)$ solves

$$
\begin{array}{cl}
\partial_{t} g-\frac{1}{2} \partial_{y}^{2} g=0 & \text { for } t>0, y \in\left(0, h_{0}\right), \\
g(x, 0, t)=g\left(x, h_{0}, t\right)=0 & \text { for } t>0, \\
g(x, y, 0)=u_{0}(x, y)-u_{0}(x, 0) & \text { for } y \in\left(0, h_{0}\right), t=0 .
\end{array}
$$

Remark. As we will see later, the Laplace transform of $w$ is given by

$$
\mathcal{L} w(s)=\int_{0}^{\infty} e^{-s t} w(t) d t=\frac{2 \tanh \left(h_{0} \sqrt{2 s}\right)}{\sqrt{2 s}} .
$$

For $h_{0}=\infty$,

$$
w(t)=\left(\frac{2}{\pi t}\right)^{1 / 2}, \quad \text { and } \quad \mathcal{L} w(s)=\left(\frac{2}{s}\right)^{1 / 2} .
$$

In this case, $\partial_{t}^{w}$ is precisely $\sqrt{2} \partial_{t}^{1 / 2}$, the standard Caputo derivative of order $1 / 2$ (see for instance [Die10]), and equation (1.8) becomes the Basset differential equation [Bas87].

Finally we conclude this section with two remarks on generalizations of Theorem 1.1.

Remark 1.5 (Other scalings). The width of the spine and teeth may be scaled in different ways to obtain the same limiting process as in Theorem 1.1. Explicitly, let

$$
\tilde{\Omega}_{\varepsilon}=\left\{(x, y) \in \mathbb{R}^{2} \mid-w_{S}(\varepsilon)<y<h_{0} \mathbf{1}_{B\left(\varepsilon \mathbb{Z}, w_{T}(\varepsilon) / 2\right)}(x)\right\},
$$

where $w_{S}(\varepsilon)$ and $w_{T}(\varepsilon)$ denote the width of the spine and teeth respectively. We claim that Theorem 1.1 still holds (with the same limiting process), provided

$$
\lim _{\varepsilon \rightarrow 0} \frac{w_{T}}{\varepsilon w_{S}(\varepsilon)}=\alpha \in(0, \infty), \quad \text { and } \quad \lim _{\varepsilon \rightarrow 0} w_{S}(\varepsilon)=0 .
$$

The proof of Theorem 1.1 needs to be modified slightly to account for this more general statement. These modifications are described in Section 3.7, below.

In the degenerate case when $\alpha=0$, the process $Z^{\varepsilon}$ rarely enters the teeth and the limiting behavior is simply that of a horizontal Brownian motion. On the other hand, if $\alpha=\infty$, then the process $Z^{\varepsilon}$ enters the teeth too often, and the limiting behavior is simply that of a vertical, doubly reflected, Brownian motion.

Remark 1.6 (Higher dimensional models). Theorem 1.1 can also be extended to analogous higher-dimensional models. For example, let $\Omega_{\varepsilon}^{\prime} \subseteq \mathbb{R}^{3}$ be a three dimensional "brush", defined by

$$
\Omega_{\varepsilon}^{\prime} \stackrel{\text { def }}{=} \bigcup_{k \in \mathbb{Z}}\left(Q_{k} \cup T_{k}\right)
$$

Here $Q_{k}$ and $T_{k}$ are defined by

$$
\begin{aligned}
Q_{k} & \stackrel{\text { def }}{=}\left(\varepsilon k-\frac{\varepsilon}{2}, \varepsilon k+\frac{\varepsilon}{2}\right) \times\left(-\frac{\varepsilon}{2}, \frac{\varepsilon}{2}\right) \times[-\varepsilon, 0), \\
T_{k} & \stackrel{\text { def }}{=}\left\{\left(x_{1}, x_{2}, x_{3}\right) \in \mathbb{R}^{3} \mid\left(\left(x_{1}-\varepsilon k\right)^{2}+x_{2}^{2}\right)^{1 / 2} \leqslant r \varepsilon^{3 / 2}, \quad x_{3} \in\left[0, h_{0}\right)\right\} .
\end{aligned}
$$

In this case, the spine is the set $\cup_{k} \overline{Q_{k}}$, an infinite rectangular cylinder; the cylindrical teeth $T_{k}$ are spaced $O(\varepsilon)$ apart and have radius $r \varepsilon^{3 / 2}>0$. If $Z^{\varepsilon}$ is a Brownian motion in this domain with normal reflection at the boundary, then one obtains an 
analogous scaling limit as $\varepsilon \rightarrow 0$. The $O\left(\varepsilon^{3 / 2}\right)$ scaling of the radius of the teeth is chosen so that the ratio

$$
\frac{2 \operatorname{Vol}\left(Q_{k}\right)}{\operatorname{Area}\left(\overline{Q_{k}} \cap \overline{T_{k}}\right)}=\frac{2}{\pi r^{2}}
$$

is independent of $\varepsilon$ - this constant ratio plays the same role as the constant $2 / \alpha$ in the comb-shaped domain $\Omega_{\varepsilon}$. While our proof of Theorem 1.1 extends to this higher-dimensional version in a straight-forward way, the added modifications are technical. Thus, for simplicity and clarity of presentation, we only focus only on the comb-shaped domain as defined above for Theorem 1.1.

1.2. Anomalous Diffusion in Comb-Shaped Graphs. We now turn our attention to comb-shaped graphs, with the intention of studying a simpler version of the model in Section 1.1 and of relating it to other work on trapped random walks. Related random walk models on comb-shaped discrete graphs have been studied by several authors, including [BZ03, Ber06, CCFR09, CCFR11]. In each of these works, a limit process is obtained which involves a Brownian motion time-changed by the local time of an independent Brownian motion. One difference between these other works and Theorem 1.7 below is that the limiting processes in our result involves Brownian motion with sticky reflections, a consequence of the gluing condition described below. More closely related to our model are the works [BAČ $07, \mathrm{BAC}+15]$, especially Section 3.2 of [BAC+15], where the trapping and drift of the random walk plays a role that is similar to our gluing condition. In Section 5.2 below, we will use the framework in $[\mathrm{BAC}+15]$ for an alternate proof of our result in this simpler setting, illuminating the relationship between these models. Nevertheless, the analyses in these other works do not apply to the comb-shaped domains considered in the previous Section 1.1, where the boundary local time of the diffusion process (pre-limit) plays an essential role.

We consider the infinite connected comb-shaped graph, $\mathcal{C}_{\varepsilon} \subset \mathbb{R}^{2}$, be defined by

$$
\mathcal{C}_{\varepsilon}=(\mathbb{R} \times\{0\}) \cup\left(\varepsilon \mathbb{Z} \times\left[0, h_{0}\right)\right) .
$$

We think of $\mathbb{R} \times\{0\}$ as the spine of $\mathcal{C}_{\varepsilon}$, and $\varepsilon \mathbb{Z} \times\left[0, h_{0}\right)$ as the infinite collection of teeth. The teeth meet the spine at the junction points $J_{\varepsilon} \subseteq \mathcal{C}_{\varepsilon}$ defined by

$$
J_{\varepsilon} \stackrel{\text { def }}{=}(\varepsilon \mathbb{Z}) \times\{0\},
$$

and is depicted in Figure 2.

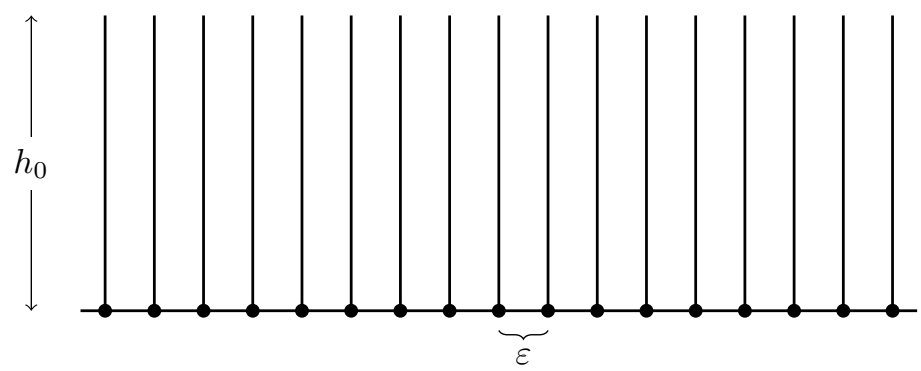

Figure 2. Image of the comb-shaped graph $\mathcal{C}_{\varepsilon}$. The teeth are spaced $\varepsilon$ apart and have height $h_{0}$. 
Let $Z^{\varepsilon}=\left(X^{\varepsilon}, Y^{\varepsilon}\right)$ be a diffusion on $\mathcal{C}_{\varepsilon}$ such that away from the junction points $J_{\varepsilon}$, the process $Z^{\varepsilon}$ is a standard Brownian motion. If $h_{0}<\infty$, we reflect $Z^{\varepsilon}$ at the ends of the teeth. At the junction points, we specify a "gluing condition" that dictates $Z^{\varepsilon}$ enters the teeth with probability $\alpha \varepsilon /(2+\alpha \varepsilon)$, and stays in the spine with probability $2 /(2+\alpha \varepsilon)$. One can formulate this precisely by requiring the local time balance

$$
L_{t}^{X^{\varepsilon}}\left(J_{\varepsilon}\right)=\frac{2}{2+\alpha \varepsilon} L_{t}^{Z^{\varepsilon}}\left(J_{\varepsilon}\right), \quad L_{t}^{Y^{\varepsilon}}\left(J_{\varepsilon}\right)=\frac{\varepsilon}{2+\alpha \varepsilon} L_{t}^{Z^{\varepsilon}}\left(J_{\varepsilon}\right),
$$

at the junction points, and we describe this further in Section 4. Alternately, one can make the gluing condition precise by using the excursion decomposition of $Z^{\varepsilon}$, and we do this in Section 5.

Clearly the mechanics of the above diffusion on the comb-shaped graph $\mathcal{C}_{\varepsilon}$ shows that it is a simplified model of the diffusion on the comb-shaped domain $\Omega_{\varepsilon}$. Our main result in this section shows convergence of $Z^{\varepsilon}$ to the same limit process as that in Theorem 1.1.

Theorem 1.7. Let $\left(\mu^{\varepsilon}\right)$ be sequence of probability measures on $\mathcal{C}_{\varepsilon}$ which converge weakly to a probability measure $\mu$ on $\Omega_{0} \stackrel{\text { def }}{=} \mathbb{R} \times\left[0, h_{0}\right]$. Let $Z^{\varepsilon}$ be the above graph diffusion with initial distribution $\mu^{\varepsilon}$. Then, as $\varepsilon \rightarrow 0$, the processes $Z^{\varepsilon}$ converge weakly to the same limit process $Z=(X, Y)$ defined in Theorem 1.1.

The proof of Theorem 1.7 is technically and conceptually much simpler than that of Theorem 1.1, and is presented in Section 4. Moreover, the excursion decomposition of $Z^{\varepsilon}$ on the comb-shaped graph $\mathcal{C}_{\varepsilon}$ allows for an elegant proof using time changes and the trapped Brownian motion framework in $[\mathrm{BAC}+15]$. We present this approach in Section 5.

The process process $Z^{\varepsilon}$ on the comb-shaped graph $\mathcal{C}_{\varepsilon}$ is closely related to a model of fluid flow in fissured media, where trapping in microscopic regions of low permeability yields a macroscopic anomalous diffusive effect. Explicitly, consider medium composed of two materials: a set of blocks, where the permeability is relatively low, and fissures where the permeability is relatively high (see for instance [ADH90,SW91,BLM96]). Assuming that the region occupied by the fissures is connected and that the blocks are arranged periodically, the fluid flow in this situation is modeled by the equation

$$
\partial_{t} u^{\varepsilon}-\nabla \cdot\left(a^{\varepsilon} \nabla u^{\varepsilon}\right)=f, \quad a^{\varepsilon}(x)=\mathbf{1}_{F}\left(\frac{x}{\varepsilon}\right) a\left(\frac{x}{\varepsilon}\right)+\varepsilon^{2} \mathbf{1}_{B}\left(\frac{x}{\varepsilon}\right) A\left(\frac{x}{\varepsilon}\right) .
$$

Here $a, A$ are uniformly elliptic matrices representing the permeability in the fissures and blocks respectively, and $F, B$ denote the region occupied by the blocks and fissures respectively. For this linear model, Clark [Cla98] proved that as $\varepsilon \rightarrow 0$, the functions $u^{\varepsilon}$ two-scale converges to a function $U=U(x, y, t)$ that satisfies a coupled system, called the double-porosity model, in which the fluid in the fissures is driven in a non-local manner by the fluid in the blocks.

To understand this model probabilistically, one could study a diffusion $\tilde{Z}^{\varepsilon}$ whose generator is $\nabla \cdot a^{\varepsilon} \nabla$. Inside the fissures, the process $\tilde{Z}^{\varepsilon}$ diffuses freely until it hits the boundary of a block. Upon hitting a block boundary, the contrast between the block and fissure permeabilities dictates that $\tilde{Z}^{\varepsilon}$ enters the blocks with probability $O(\varepsilon)$, and remains in the fissures with probability $1-O(\varepsilon)$. Since the blocks have diameter $O(\varepsilon)$, and the permeability there is $O\left(\varepsilon^{2}\right)$, the excursions of $\tilde{Z}^{\varepsilon}$ into the blocks take $O(1)$ amount of time. These characteristic features are exactly captured by 
the above comb model: the spine plays the role of the fissures and the teeth play the role of the blocks (rescaled to have size 1), and our gluing condition dictates that $Z^{\varepsilon}$ enters the teeth with probability $O(\varepsilon)$.

Plan of this paper. The rest of the paper is organized as follows. We begin by describing the limit process $Z$, and study its basic properties in Section 2. Next, in Section 3 we prove Theorem 1.1 and all the required lemmas. In Section 4 we prove Theorem 1.7 on the comb-shaped graph $\mathcal{C}_{\varepsilon}$. The proof is similar to that of Theorem 1.1, but the technicalities are much simpler. Finally, in Section 4 we provide an alternate proof of Theorem 1.7 using the trapped Brownian motion framework in $[\mathrm{BAC}+15]$.

\section{The Limit Process.}

Before proving our main results in this paper, we give a more thorough description of the limit process $Z=(X, Y)$. There are two canonical constructions of this process. The first, relatively well-known construction involves directly writing $Y$ as a time-changed Brownian motion, and this is presented in Section 2.1. The second construction involves a characterization using the generator. While the technicalities using this second approach are more involved, they relate to the PDE analogue and immediately yield Corollary 1.3.

Remark 2.1. The process $Z$ depends on the parameters $\alpha>0$, and $h_{0} \in(0, \infty]$. To simplify the presentation, we will subsequently assume $h_{0}=1$. The case $h_{0}=\infty$ may be handled by replacing the normal reflection at 1 with a diffusion on the semi-infinite interval $(0, \infty)$.

2.1. Construction via Time Changes. We begin by constructing the limit process $Z$ using a time-changed Brownian motion. To construct the process $Y$, let $\bar{B}_{t}$ be a standard doubly reflected Brownian motion on the interval $(0,1)$. (Recall that in Remark 2.1 we assumed $h_{0}=1$ for simplicity.) Let $L_{s}^{\bar{B}}(0)$ be the local time of $\bar{B}$ at 0 , and define

Let $T$, defined by

$$
\varphi(s) \stackrel{\text { def }}{=} s+\frac{2}{\alpha} L_{s}^{\bar{B}}(0), \quad s \geqslant 0 .
$$

$$
T(t)=T_{t} \stackrel{\text { def }}{=} \varphi^{-1}(t)=\inf \{s \geqslant 0 \mid \varphi(s) \geqslant t\},
$$

denote the inverse of $\varphi$. Since $\varphi$ is strictly increasing, note that $T$ is continuous. Thus the process $Y$, defined by

$$
Y_{t} \stackrel{\text { def }}{=} \bar{B}_{T_{t}}
$$

is a continuous process on $[0,1]$. Clearly, on any interval of time where $Y$ remains inside the interval $(0,1]$, trajectories of $Y$ and $\bar{B}$ are identical. When $Y$ hits 0 , however, the trajectories are slowed down on account of the time change $T$. The behavior at 0 is known as a sticky reflection with parameter $1 / \alpha$ at 0 , and we refer the reader to [IM74, 14, §5.7] or the original papers of Feller [Fel52, Fel54] for more details.

Clearly once the process $Y$ is known, the process $X$ can be recovered using (1.2), reproduced here for convenience:

$$
X_{t} \stackrel{\text { def }}{=} \bar{W}_{\frac{2}{\alpha}} L_{t}^{Y}(0)
$$


Here $\bar{W}$ is standard one dimensional Brownian motion that is independent of $\bar{B}$. Intuitively, we think of $\mathbb{R} \times\{0\}$ as the spine of the limiting comb, and $\mathbb{R} \times\left(0, h_{0}\right]$ as the continuum of teeth. The process $T_{t}$ may be interpreted as the time accumulated in the teeth, and $\frac{2}{\alpha} L_{t}^{Y}(0)$ is the time accumulated in the spine.

2.2. The SDE Description. We now describe the process $Z=(X, Y)$ via a system of SDEs. Let $W$ and $B$ be two independent standard one dimensional Brownian motions. We claim that the process $Z$ can be characterized as the solution of the system of SDEs

$$
\begin{gathered}
d X_{t}=\mathbf{1}_{\left\{Y_{t}=0\right\}} d W_{t}, \\
d Y_{t}=\mathbf{1}_{\left\{Y_{t} \neq 0\right\}} d B_{t}-d L_{t}^{Y}(1)+d L_{t}^{Y}(0), \\
\alpha \mathbf{1}_{\left\{Y_{t}=0\right\}} d t=2 d L_{t}^{Y}(0),
\end{gathered}
$$

with initial distribution $\mu$. Existence of a process $Z$ satisfying (2.3a)-(2.3c) can be shown abstractly using the Hille-Yosida theorem, and we refer the reader to [Coh18] for the details. Instead, we will show existence by showing that the process $Z$ constructed in the previous section is a solution to (2.3a)-(2.3c).

Lemma 2.2. The process $Z=(X, Y)$ defined by $(2.2 \mathrm{a})-(2.2 \mathrm{~b})$ is a weak solution to the system $(2.3 \mathrm{a})-(2.3 \mathrm{c})$.

The proof of Lemma 2.2 boils down to an SDE characterization of sticky Brownian motion that was recently shown by Engelbert and Peskir [EP14]. We remark that in [EP14] the authors also show weak uniqueness of the appropriate SDE. While we present the proof of existence below, we refer the reader to [EP14] for the proof of uniqueness.

Proof. By the Tanaka formula we have

$$
\bar{B}_{t}=\tilde{B}_{t}+L_{t}^{\bar{B}}(0)-L_{t}^{\bar{B}}(1),
$$

where $\tilde{B}$ is a Brownian motion. Since $T_{t}$ is a continuous and increasing time change, $\tilde{B}_{T_{t}}$ is still a continuous martingale, $L_{t}^{Y}(0)=L_{T_{t}}^{\bar{B}}(0)$ and $L_{t}^{Y}(1)=L_{T_{t}}^{\bar{B}}(1)$. Note first

$$
\alpha \int_{0}^{t} \mathbf{1}_{\left\{Y_{s}=0\right\}} d s=\alpha \int_{0}^{t} \mathbf{1}_{\left\{\bar{B}_{T_{s}}=0\right\}} d \varphi\left(T_{s}\right)=\alpha \int_{0}^{T_{t}} \mathbf{1}_{\left\{\bar{B}_{s}=0\right\}} d \varphi(s) .
$$

Then since $\left\{t \mid \bar{B}_{t}=0\right\}$ has Lebesgue measure 0 and $L_{t}^{\bar{B}}$ only increases on this set, we decompose $\alpha \varphi(s)=\alpha s+2 L_{s}^{\bar{B}}$ to obtain

$$
\alpha \int_{0}^{T_{t}} \mathbf{1}_{\left\{\bar{B}_{s}=0\right\}} d \varphi(s)=2 \int_{0}^{T_{t}} \mathbf{1}_{\left\{\bar{B}_{s}=0\right\}} d L_{s}^{\bar{B}}(0)=2 L_{T_{t}}^{\bar{B}}(0)=2 L_{t}^{Y}(0),
$$

which implies (2.3c). Notice that since $(2 / \alpha) L_{t}^{Y}(0)$ is independent of $\bar{W}, X_{t}$ is a martingale with quadratic variation

$$
\langle X\rangle_{t}=\frac{2}{\alpha} L_{t}^{Y}(0)
$$

In addition we have

$$
\left\langle\tilde{B}_{T}\right\rangle_{t}=T_{t} .
$$

Thus, for the process $B$ defined by

$$
B_{t} \stackrel{\text { def }}{=} \tilde{B}_{T_{t}}+\bar{W}_{\frac{2}{\alpha} L_{t}^{Y}(0)},
$$


we have $\langle B\rangle_{t}=t$. For the filtration, we let

$$
\mathcal{G}_{t}=\sigma\left(\mathcal{N} \cup \mathcal{F}_{T_{t}}^{\bar{B}} \cup \mathcal{F}_{t}^{X}\right)
$$

where $\mathcal{N}$ denotes the collection of all $\mathcal{F}_{\infty}^{(\bar{B}, \bar{W})}$-null sets. Since $\bar{B}$ and $\bar{W}$ are independent, it is easy to see that for all $s \geqslant 0, X_{t}-X_{s}$ is independent of $\mathcal{G}_{s}$, and both $\tilde{B}_{T_{t}}$ and $X_{t}$ are $\mathcal{G}$-martingales. Thus, $B$ is also a $\mathcal{G}$-martingale, and by Lévy's criterion must be a Brownian motion.

Now (2.3a)-(2.3b) follow from (2.3c), (2.8) and the fact that

$$
\int_{0}^{t} \mathbf{1}_{\left\{Y_{s}=0\right\}} d \tilde{B}_{T_{s}}=0 \text { and } \int_{0}^{t} \mathbf{1}_{\left\{Y_{s} \neq 0\right\}} d X_{s}=0 .
$$

2.3. Computing the Generator (Lemma 2.3). We now compute the generator of $Z$. In the teeth (when $y>0$ ) this is a standard calculation with Itô's formula. In the spine (when $y=0$ ), however, one needs to estimate the time spent in the spine. We state this precisely and carry out the details here.

Lemma 2.3. Let $\Omega_{0}=\mathbb{R} \times[0,1)$, and define the operator $A$ by

$$
A \stackrel{\text { def }}{=} \frac{1}{2} \partial_{y}^{2} \text {. }
$$

Define the domain of $A$, denoted by $\mathcal{D}(A)$, to be the set of all functions $g \in C_{0}\left(\Omega_{0}\right) \cap$ $C_{b}^{2}\left(\Omega_{0}\right)$ such that

$$
\partial_{y} g(x, 1)=0, \quad \text { and } \quad \partial_{x}^{2} g(x, 0)+\alpha \partial_{y} g(x, 0)=\partial_{y}^{2} g(x, 0) .
$$

The generator of the process $Z$ (defined by (2.2a)-(2.2b)) is the operator $A$ with domain $\mathcal{D}(A)$.

Proof. Choose $g \in \mathcal{D}(A)$ and apply Itô's formula to obtain

$$
\begin{aligned}
g\left(X_{t}, Y_{t}\right)=g\left(X_{0}, Y_{0}\right) & +\int_{0}^{t} \partial_{x} g\left(X_{s}, Y_{s}\right) d X_{s}+\int_{0}^{t} \partial_{y} g\left(X_{s}, Y_{s}\right) d Y_{s} \\
& +\frac{1}{\alpha} \int_{0}^{t} \partial_{x}^{2} g\left(X_{s}, Y_{s}\right) d L_{s}^{Y}(0)+\frac{1}{2} \int_{0}^{t} \partial_{y}^{2} g\left(X_{s}, Y_{s}\right) d T_{s} .
\end{aligned}
$$

Taking expectations gives

$$
\begin{aligned}
\boldsymbol{E}^{(x, y)}\left[g\left(X_{t}, Y_{t}\right)-g(x, y)\right] & =\boldsymbol{E}^{(x, y)}\left[\int_{0}^{t} \partial_{y} g\left(X_{s}, Y_{s}\right) d Y_{s}\right] \\
& +\boldsymbol{E}^{(x, y)}\left[\frac{1}{\alpha} \int_{0}^{t} \partial_{x}^{2} g\left(X_{s}, Y_{s}\right) d L_{s}^{Y}(0)+\frac{1}{2} \int_{0}^{t} \partial_{y}^{2} g\left(X_{s}, Y_{s}\right) d T_{s}\right] .
\end{aligned}
$$

Now for $y \in(0,1)$ we know $Y$ is a Brownian motion before it first hits 0 or 1 , and hence $\lim _{t \rightarrow 0} \boldsymbol{P}^{y}\left(L_{t}^{Y}(0) \neq 0\right)=0$. Moreover by definition of $T$, we know $T_{t}=t$ when $\left\{L_{t}^{Y}=0\right\}$. Consequently

$$
\lim _{t \rightarrow 0} \boldsymbol{E}^{(x, y)}\left[\frac{g\left(X_{t}, Y_{t}\right)-g(x, y)}{t}\right]=\frac{1}{2} \partial_{y}^{2} g(x, y) .
$$

For $y=1$ we note

$$
\lim _{t \rightarrow 0} \boldsymbol{E}^{(x, 1)}\left[\frac{g\left(X_{t}, Y_{t}\right)-g(x, y)}{t}\right]
$$




$$
=\frac{1}{2} \partial_{y}^{2} g(x, 1)+\lim _{t \rightarrow 0} \boldsymbol{E}^{(x, 1)}\left[\frac{1}{t} \int_{0}^{t} \partial_{y} g\left(X_{s}, Y_{s}\right) d Y_{s}\right] .
$$

By (2.4) we know $\boldsymbol{E}^{(x, 1)} L_{t}^{Y}(1)=O(\sqrt{t})$, and hence the right hand side of (2.12) is finite if and only if $\partial_{y} g(x, 1)=0$.

Finally, we compute the generator on the spine $y=0$. First we show that if we start $Y$ at 0 then for a short time it spends "most" of the time at 0 . More precisely we claim

$$
\lim _{t \rightarrow 0} \boldsymbol{E}^{0}\left[\frac{T_{t}}{t}\right]=0 .
$$

Here we clarify that the 0 superscript on $\boldsymbol{E}$ refers to the initial distribution of the process $\bar{B}$, where as the double superscript $\boldsymbol{E}^{(x, y)}$, or measure superscript $\boldsymbol{E}^{\mu}$ used earlier refers to the initial distribution of the joint process $Z=(X, Y)$.

Let $M_{t}$ be the running maximum of $\tilde{B}$. Note that since $L^{\bar{B}}=L^{\tilde{B}}$ on $\left\{M_{t}<1\right\}$, we have

$$
\begin{aligned}
\boldsymbol{P}^{0}\left(L_{t}^{\bar{B}}(0) \leqslant r\right) \leqslant \boldsymbol{P}^{0}\left(L_{t}^{\tilde{B}}(0)\right. & \leqslant r)+\boldsymbol{P}^{0}\left(M_{t}>1\right) \\
& =1-2 \boldsymbol{P}^{0}\left(r<\tilde{B}_{t}<1\right) \leqslant \sqrt{\frac{2}{\pi}}\left(\frac{r}{\sqrt{t}}+\sqrt{t} e^{-\frac{1}{2 t}}\right) .
\end{aligned}
$$

Thus,

$$
\begin{aligned}
\boldsymbol{E}^{0}\left[\frac{T_{t}}{t}\right] & =\int_{0}^{1} \boldsymbol{P}^{0}\left(T_{t}>s t\right) d s=\int_{0}^{1} \boldsymbol{P}^{0}\left(s t+2 L_{s t}^{\bar{B}}(0) \leqslant t\right) d s \\
& =\int_{0}^{1} \boldsymbol{P}^{0}\left(L_{s t}^{\bar{B}}(0) \leqslant \frac{(1-s) t}{2}\right) d s \leqslant \int_{0}^{1} \sqrt{\frac{2}{\pi}}\left(\frac{2(1-s)}{\sqrt{s}} \sqrt{t}+\sqrt{s t} e^{-1 / 2 s t}\right) d s \\
& \leqslant C \sqrt{t} .
\end{aligned}
$$

With this estimate, we can now compute generator on the spine. Using equation (2.13) we see

$$
\boldsymbol{E}^{0}\left[\frac{L_{t}^{Y}(0)}{t}\right]=\boldsymbol{E}^{0}\left[\frac{L_{T_{t}}^{\bar{B}}(0)}{t}\right]=\frac{\alpha}{2} \boldsymbol{E}^{0}\left[\frac{t-T_{t}}{t}\right] \stackrel{t \rightarrow 0}{\longrightarrow} \frac{\alpha}{2} .
$$

Using (2.4) we have,

$$
\boldsymbol{E}^{0}\left[\frac{Y_{t}}{t}\right]=\boldsymbol{E}^{0}\left[\frac{\bar{B}_{T_{t}}}{t}\right]=\boldsymbol{E}^{0}\left[\frac{\tilde{B}_{T_{t}}+L_{T_{t}}^{\bar{B}}(0)-L_{T_{t}}^{\bar{B}}(1)}{t}\right] .
$$

Since $T_{t} \leqslant t$, the third term tends to 0 and using the modulus of continuity for Brownian motion the first term does as well. Therefore we also have

$$
\boldsymbol{E}^{0}\left[\frac{Y_{t}}{t}\right] \stackrel{t \rightarrow 0}{\longrightarrow} \frac{\alpha}{2}
$$

Thus using (2.13), (2.14) and (2.15) in equation (2.11) gives

$$
\lim _{t \rightarrow 0} \frac{1}{t} \boldsymbol{E}^{(x, y)}\left[g\left(X_{t}, Y_{t}\right)-g(x, y)\right]=\frac{\alpha}{2} \partial_{y} g(x, 0)+\frac{1}{2} \partial_{x}^{2} g(x, 0)+0,
$$

finishing the proof. 
2.4. PDE Homogenization (Corollaries 1.2, 1.3, and Proposition 1.4). Once the generator of $Z$ is known, the behavior of the variance (Corollary 1.2) and PDE homogenization result (Corollary 1.3) can be deduced quickly.

Proof of Corollary 1.2. We first assume $h_{0}=1$ as in Remark 2.1. Using Theorem 1.1 and (2.7) we see

$$
\lim _{\varepsilon \rightarrow 0} \boldsymbol{E}^{(x, 0)}\left|X_{t}^{\varepsilon}-x\right|^{2}=\boldsymbol{E}^{(x, 0)}\left|X_{t}-x\right|^{2}=\frac{2}{\alpha} \boldsymbol{E}^{0} L_{t}^{Y}(0) .
$$

Now equation (1.3a) follows from (2.14).

For the long time limit (when $h_{0}=1$ ) we note that by ergodicity of $\bar{B}$, we know that $\boldsymbol{E}^{0}\left|L_{t}^{\bar{B}} / t-1 / 2\right| \rightarrow 0$ as $t \rightarrow \infty$. Thus using (2.1) we must have

$$
\lim _{t \rightarrow \infty} \boldsymbol{E}^{0}\left|\frac{T(t)}{t}-\frac{\alpha}{\alpha+1}\right|=0 .
$$

Consequently,

$$
\boldsymbol{E}^{0}\left(\frac{L_{t}^{Y}(0)}{t}\right)=\boldsymbol{E}^{0}\left(\frac{L_{T_{t}}^{\bar{B}}}{t}\right)=\frac{\alpha}{2} \boldsymbol{E}^{0}\left(\frac{t-T_{t}}{t}\right) \stackrel{t \rightarrow \infty}{\longrightarrow} \frac{\alpha}{2(\alpha+1)},
$$

and together with (2.16) this implies (1.3b). This finishes the proof of (1.3a) and $(1.3 \mathrm{~b})$ when $h_{0}=1$. The case for arbitrary finite $h_{0}$ is similar.

When $h_{0}=\infty$, the process $Y$ is a sticky Brownian motion on the half line, and the distribution of $L_{t}^{Y}(0)$ can be computed explicitly. Namely (see for instance [How07]) we have

$$
\frac{2}{\alpha} L_{t}^{Y}(0)=\int_{0}^{t} \mathbf{1}_{\left\{Y_{s}=0\right\}} d s \sim \frac{2|N|}{\alpha}\left(t+\frac{N^{2}}{\alpha^{2}}\right)^{1 / 2}-\frac{2 N^{2}}{\alpha^{2}},
$$

where $N$ is the standard normal. Taking expectations and using (2.16) immediately yields (1.3a) and (1.4), finishing the proof.

Proof of Corollary 1.3. By the Kolmogorov backward equation [Fri75, §5.6] we known that the function $u^{\varepsilon}$ (defined by $\left.(1.5 \mathrm{a})-(1.5 \mathrm{~b})\right)$ satisfies

$$
u^{\varepsilon}(z, t)=\boldsymbol{E}^{z} u_{0}\left(Z_{t}^{\varepsilon}\right) \text {. }
$$

Consequently

$$
\int_{\Omega_{\varepsilon}} u^{\varepsilon}(z, t) d \mu^{\varepsilon}(z)=\boldsymbol{E}^{\mu^{\varepsilon}} u_{0}\left(Z_{t}^{\varepsilon}\right) \stackrel{\varepsilon \rightarrow 0}{\longrightarrow}=\boldsymbol{E}^{\mu} u_{0}\left(Z_{t}\right),
$$

by Theorem 1.1. Thus, if we set

$$
u(z, t)=\boldsymbol{E}^{z} u_{0}\left(Z_{t}\right)
$$

we see that (1.6) holds.

It only remains to verify that $u$ satisfies $(1.7 \mathrm{a})-(1.7 \mathrm{~d})$ hold. To see this, recall that the function $u$ defined by $(2.18)$ belongs to $C(0, \infty ; \mathcal{D}(A))$ and satisfies the Kolmogorov equations

$$
\begin{array}{cl}
\partial_{t} u-A u=0 & t>0, \\
u(\cdot, t)=u_{0} & \text { when } t=0 .
\end{array}
$$

The first equation above implies (1.7a) by definition of $A$ (equation (2.9)). Equations (1.7b) and (1.7c) follow from the fact that $u(\cdot, t) \in \mathcal{D}(A)$ for all $t>0$, and equation $(1.7 \mathrm{~d})$ follows from the second equation above. 
We now obtain evolution equations for the slice of $u$ at $y=0$, as stated in Proposition 1.4.

Proof of Proposition 1.4. Let $u_{1}=u-g$, and observe that $u_{1}$ satisfies (1.7a) with initial data $u_{1}(x, y, 0)=u_{0}(x, 0)=v_{0}(x)$, and boundary conditions

$$
u_{1}(x, 0, t)=u(x, 0, t)=v(x, t) \quad \text { and } \quad \partial_{y} u_{1}(x, 1, t)=0 .
$$

(Recall that in Remark 2.1 we have already set $h_{0}=1$ for simplicity.) We now treat $x$ as a parameter, and solve (1.7a) using separation of variables (in $y, t)$ with boundary conditions (2.19). A direct calculation shows

$$
\partial_{y} u_{1}(x, 0, t)=-\partial_{t}^{w} v
$$

and hence

$$
\partial_{y} u(x, 0, t)=-\partial_{t}^{w} v(x, t)+\partial_{y} g(x, 0, t) .
$$

Now for $t>0$ using equation (1.7a) and (1.7b) and continuity of second derivatives of $u$ up to $y=0$ we see

$$
\partial_{t} v(x, t)=\frac{\alpha}{2} \partial_{y} u(x, 0, t)+\frac{1}{2} \partial_{x}^{2} v(x, t) .
$$

Using (2.21) and (2.22) yields (1.8) as claimed.

Remark 2.4. For brevity, we have suppressed the explicit separation of variables calculation deriving (2.20). One can avoid this calculation by using the Laplace transform as follows. Following standard convention, we will denote the Laplace transform of a function using an upper case letter using the variable $s$, instead of $t$. Explicitly, given a function $f$, we define its Laplace transform, denoted by $F$ or $\mathcal{L} f$, by

$$
F(s) \stackrel{\text { def }}{=} \mathcal{L} f(s)=\int_{0}^{\infty} e^{-s t} f(t) d t
$$

For functions that depend on both space and time variables, the Laplace transform will only be with respect to the time variable.

Taking the Laplace transform of $u_{1}$ yields the ODE in the variable $y$

$$
s U_{1}-v_{0}-\frac{1}{2} \partial_{y}^{2} U_{1}=0,
$$

with boundary conditions $U_{1}(x, 0, s)=V(x, s)$, and $\partial_{y} U_{1}(x, 1, s)=0$. Solving this ODE yields

$$
U_{1}(x, y, s)=\frac{v_{0}}{s}+\left(\frac{1}{1+e^{2 \sqrt{2 s}}}\right)\left(V-\frac{v_{0}}{s}\right)\left[e^{y \sqrt{2 s}}+e^{\sqrt{2 s}(2-y)}\right],
$$

and hence

$$
\partial_{y} U_{1}(x, 0, s)=-\sqrt{2 s}\left(V-\frac{v_{0}}{s}\right) \tanh \sqrt{2 s}=-\frac{2 \tanh \sqrt{2 s}}{\sqrt{2 s}}\left(s V-v_{0}\right) .
$$

Choosing $w$ to be a function with Laplace transform (1.9), implies (2.20) as claimed. 


\section{Comb-Shaped Domains (Theorem 1.1).}

We now turn to the proof of Theorem 1.1. Recall that $Z_{t}^{\varepsilon,+}=\pi_{\varepsilon}\left(Z_{t}^{\varepsilon}\right)=$ $\left(X_{t}^{\varepsilon}, \max \left(Y_{t}^{\varepsilon}, 0\right)\right)$. The main ingredients in the proof are the following lemmas.

Lemma 3.1. Let $Z^{\varepsilon}=\left(X^{\varepsilon}, Y^{\varepsilon}\right)$ be the reflected Brownian motion on the combshaped domain $\Omega_{\varepsilon}$, as described in Theorem 1.1. Then, for any $T>0$, the family of processes $Z^{\varepsilon}$ is tight in $C\left([0, T] ; \mathbb{R}^{2}\right)$.

Lemma 3.2. Let $A$ be the generator defined in (2.9), with domain $\mathcal{D}(A)$. Weak uniqueness holds for the martingale problem for $A$.

Lemma 3.3. If $f \in \mathcal{D}(A)$, and $K \subset \Omega_{0}$ is compact, then

$$
\lim _{\varepsilon \rightarrow 0} \sup _{z \in K \cap \Omega_{\varepsilon}} \boldsymbol{E}^{z}\left(f\left(Z_{t}^{\varepsilon,+}\right)-f\left(Z_{0}^{\varepsilon,+}\right)-\int_{0}^{t} A f\left(Z_{s}^{\varepsilon,+}\right) d s\right)=0 .
$$

Momentarily postponing the proof of these lemmas, we prove Theorem 1.1.

Proof of Theorem 1.1. Suppose first $Z^{\varepsilon,+} \rightarrow Z^{\prime}$ weakly along some subsequence. We claim $Z^{\prime}$ should be a solution of the martingale problem for $A$ with initial distribution $\mu$. To see this set

$$
M_{t}^{\varepsilon}=f\left(Z_{t}^{\varepsilon,+}\right)-f\left(Z_{0}^{\varepsilon,+}\right)-\int_{0}^{t} A f\left(Z_{r}^{\varepsilon,+}\right) d r
$$

and observe

$$
\boldsymbol{E}^{\mu^{\varepsilon}}\left(M_{t}^{\varepsilon} \mid \mathcal{F}_{s}\right)=M_{s}^{\varepsilon}+\boldsymbol{E}^{Z_{s}^{\varepsilon}}\left(M_{t-s}^{\varepsilon}\right)
$$

by the Markov property. Using Lemma 3.3, and taking limits along this subsequence, the last term on the right vanishes. Since this holds for all $f \in \mathcal{D}(A)$ and $\mathcal{D}(A)$ is dense in $C_{0}\left(\Omega_{0}\right), Z^{\prime}$ must be a solution of the martingale problem for $A$. Since $Z^{\varepsilon,+} \rightarrow Z^{\prime}$ weakly and $\pi_{\varepsilon}^{*}\left(\mu^{\varepsilon}\right) \rightarrow \mu$ weakly by assumption, we have $Z(0) \sim \mu$. By uniqueness of solutions to the martingale problem for $A$ (Lemma 3.2), the above argument shows uniqueness of subsequential limits of $Z^{\varepsilon,+}$. Combined with tightness (Lemma 3.1), and the fact that $Z$ is a solution to the martingale problem for $A$ (Lemma 2.3), this gives weak convergence as desired.

It remains to prove Lemmas 3.1-3.3. We do this in Sections 3.1, 3.2 and 3.3, below.

3.1. Proof of Tightness (Lemma 3.1). To prove tightness, we need an auxiliary lemma comparing the oscillation of trajectories in the spine to that of Brownian motion. This will also be used in the proof of Lemma 3.3.

Lemma 3.4. Let $W^{\prime}$ be a standard Brownian motion on $\mathbb{R}$ with $W^{\prime}(0)=0$. For any $T>0, \varepsilon \in(0,1 / 2], z \in \Omega_{\varepsilon}$, and any $a, \delta>0$, we have

$$
\boldsymbol{P}^{z}\left(\sup _{\substack{r, t \in[0, T] \\|t-r| \leqslant \delta}}\left|X^{\varepsilon}(t)-X^{\varepsilon}(r)\right| \geqslant a\right) \leqslant \boldsymbol{P}\left(\sup _{\substack{r, t \in[0, T] \\|t-r| \leqslant \delta}} 4\left|W^{\prime}(t)-W^{\prime}(r)\right| \geqslant a-2 \varepsilon\right) .
$$

Proof. Let

$$
\tau_{0}=\inf \left\{t \geqslant 0 \mid X^{\varepsilon}(t) \in \varepsilon\left(\mathbb{Z}+\frac{1}{2}\right)\right\},
$$

and inductively define

$$
\tau_{k+1}=\inf \left\{t \geqslant \tau_{k}|| X^{\varepsilon}(t)-X^{\varepsilon}\left(\tau_{k}\right) \mid=\varepsilon\right\},
$$


for $k \geqslant 0$. By symmetry of the domain, observe that $k \mapsto X^{\varepsilon}\left(\tau_{k}\right)$ defines a simple random walk on the discrete points $\varepsilon(\mathbb{Z}+1 / 2)$. Next, define

$$
\tau_{k}^{\prime}=\inf \left\{t \geqslant \tau_{k}|| X^{\varepsilon}(t)-X^{\varepsilon}\left(\tau_{k}\right) \mid=\varepsilon / 4\right\}, \quad k \geqslant 0 .
$$

In particular, $\tau_{k}<\tau_{k}^{\prime}<\tau_{k+1}$. At time $\tau_{k}, X^{\varepsilon}\left(\tau_{k}\right)$ is in the spine, at the midpoint between two adjacent teeth. For $t \in\left[\tau_{k}, \tau_{k}^{\prime}\right], X^{\varepsilon}(t)$ is in the spine and cannot enter the teeth, because $\left|X^{\varepsilon}(t)-x\right| \leqslant \varepsilon / 4$ where $x=X^{\varepsilon}\left(\tau_{k}\right) \in \varepsilon\left(\mathbb{Z}+\frac{1}{2}\right)$. Define the increments $\Delta_{k} X^{\varepsilon}=X^{\varepsilon}\left(\tau_{k+1}\right)-X^{\varepsilon}\left(\tau_{k}\right) \in\{-\varepsilon,+\varepsilon\}$. By the strong Markov property and symmetry of the domain, the random variables $\left\{\left(\tau_{k}^{\prime}-\tau_{k}\right)\right\}_{k} \cup\left\{\Delta X_{k}^{\varepsilon}\right\}_{k}$ are independent.

Now, suppose that $W^{\prime}(t)$ is an independent Brownian motion on $\mathbb{R}$, with $W^{\prime}(0)=$ 0 . Define another set of stopping times inductively by $\sigma_{0}=0$ and

$$
\sigma_{k+1}=\inf \left\{t \geqslant \sigma_{k}|| W^{\prime}(t)-W^{\prime}\left(\sigma_{k}\right) \mid=\varepsilon / 4\right\}, \quad k \geqslant 0 .
$$

Let $\Delta \sigma_{k}=\sigma_{k+1}-\sigma_{k}$, and $\Delta_{k} W^{\prime}=W^{\prime}\left(\sigma_{k+1}\right)-W^{\prime}\left(\sigma_{k}\right) \in\{-\varepsilon / 4, \varepsilon / 4\}$. Observe that the family of random variables

$$
\left\{\left(\sigma_{k+1}-\sigma_{k}\right), 4 \Delta W_{k}^{\prime}\right\}_{k \geqslant 0}
$$

has the same law as the family

$$
\left\{\left(\tau_{k}^{\prime}-\tau_{k}\right), \Delta X_{k}^{\varepsilon}\right\}_{k \geqslant 0}
$$

Next, define

$$
K(t)=\max \left\{k \geqslant 0 \mid \tau_{k} \leqslant t\right\},
$$

and observe that if $|t-r| \leqslant \delta$ and $0 \leqslant r \leqslant t \leqslant T$, then we must have $\tau_{K(t)}-$ $\tau_{K(r)+1} \leqslant \delta$ and thus

$$
\sum_{j=K(r)+1}^{K(t)-1}\left(\tau_{j}^{\prime}-\tau_{j}\right) \leqslant \delta, \quad \text { and } \quad \sum_{j=0}^{K(t)-1}\left(\tau_{j}^{\prime}-\tau_{j}\right) \leqslant T .
$$

In this case,

$$
\begin{aligned}
\left|X^{\varepsilon}(t)-X^{\varepsilon}(r)\right| & \leqslant 2 \varepsilon+\left|X^{\varepsilon}(K(t))-X^{\varepsilon}(K(r)+1)\right| \\
= & 2 \varepsilon+\left|\sum_{j=K(r)+1}^{K(t)-1} \Delta X_{j}^{\varepsilon}\right| \\
& \leqslant 2 \varepsilon+\sup _{0 \leqslant \ell \leqslant m}\left|\sum_{j=\ell+1}^{m-1} \Delta X_{j}^{\varepsilon}\right| \mathbf{1}_{\left\{\sum_{j=\ell+1}^{m-1}\left(\tau_{j}^{\prime}-\tau_{j}\right) \leqslant \delta\right\}} \mathbf{1}_{\left\{\sum_{j=0}^{m-1}\left(\tau_{j}^{\prime}-\tau_{j}\right) \leqslant T\right\}} .
\end{aligned}
$$

This last supremum has the same law as

$$
\begin{aligned}
\sup _{0 \leqslant \ell \leqslant m}\left|\sum_{j=\ell+1}^{m-1} 4 \Delta W_{j}^{\prime}\right| & \mathbf{1}_{\left\{\sum_{j=\ell+1}^{m-1}\left(\sigma_{j+1}-\sigma_{j}\right) \leqslant \delta\right\}} \mathbf{1}_{\left\{\sum_{j=0}^{m-1}\left(\sigma_{j+1}-\sigma_{j}\right) \leqslant T\right\}} \\
= & \sup _{0 \leqslant \ell \leqslant m} 4\left|W^{\prime}\left(\sigma_{m}\right)-W^{\prime}\left(\sigma_{\ell+1}\right)\right| \mathbf{1}_{\left\{\sigma_{m}-\sigma_{\ell+1} \leqslant \delta\right\}} \mathbf{1}_{\left\{\sigma_{m}-\sigma_{0} \leqslant T\right\}} .
\end{aligned}
$$

Since the right hand side of the above is bounded by

$$
\sup _{\substack{r, t \in[0, T] \\|t-r| \leqslant \delta}} 4\left|W^{\prime}(t)-W^{\prime}(r)\right|,
$$

we obtain (3.2). 
We now prove Lemma 3.1.

Proof of Lemma 3.1. Note first that Lemma 3.4 immediately implies that the processes $X^{\varepsilon}$ are tight. Indeed, by (3.2) we see

$$
\lim _{\delta \rightarrow 0} \limsup _{\varepsilon \rightarrow 0} \boldsymbol{P}^{\mu^{\varepsilon}}\left(\sup _{\substack{r, t \in[0, T] \\|t-r| \leqslant \delta}}\left|X^{\varepsilon}(t)-X^{\varepsilon}(r)\right| \geqslant a\right)=0 .
$$

Moreover, since $\mu^{\varepsilon}$ converge weakly to the probability measure $\mu$, the distributions of $X_{0}^{\varepsilon}$ are tight. This implies implies tightness of the processes $X^{\varepsilon}$.

For tightness of $Y^{\varepsilon}$, we note as above that the distributions of $Y_{0}^{\varepsilon}$ are already tight. In order to control the time oscillations, fix $T>0$, and let

$$
d Z^{\varepsilon}=d B_{t}+d L_{t}^{\partial \Omega_{\varepsilon}},
$$

be the semi-martingale decomposition of $Z^{\varepsilon}$ (see for instance [SV71]). Here $B=$ $\left(B_{1}, B_{2}\right)$ is a standard Brownian motion and $L^{\partial \Omega_{\varepsilon}}$ is the local time of $Z^{\varepsilon}$ on $\partial \Omega_{\varepsilon}$. Let $\omega(\delta)=\omega_{T}(\delta)$, defined by

$$
\omega(\delta)=\sup _{\substack{s, t \in[0, T] \\|t-s| \leqslant \delta}}\left|B_{2}(t)-B_{2}(s)\right|,
$$

be the modulus of continuity for $B_{2}$ over $[0, T]$. Let $[s, t] \subset[0, T]$ with $|t-s| \leqslant \delta$. If $0<Y_{r}^{\varepsilon}<1$ for all $r \in(s, t)$, then we must have

$$
\left|Y^{\varepsilon}(t)-Y^{\varepsilon}(s)\right|=\left|B_{2}(t)-B_{2}(s)\right| \leqslant \omega(\delta) .
$$

Otherwise, for some $r \in(s, t)$ either $Y_{r}=0$ or $Y_{r}=1$. Let $G_{\delta}$ be the event that $\omega(\delta)<1 / 2$; on this event $Y$ cannot hit both 0 and 1 on the interval $[s, t]$. Define

$$
\eta_{-}=\inf \left\{r>s \mid Y_{r}^{\varepsilon} \in\{0,1\}\right\}, \quad \text { and } \quad \eta_{+}=\sup \left\{r<t \mid Y_{r}^{\varepsilon} \in\{0,1\}\right\} .
$$

In this case we have

$$
\begin{aligned}
\left|Y_{t}^{\varepsilon}-Y_{s}^{\varepsilon}\right| & \leqslant \max \left(\left|Y^{\varepsilon}\left(\eta_{-}\right)-Y^{\varepsilon}(s)\right|,\left|Y^{\varepsilon}(t)-Y^{\varepsilon}\left(\eta_{+}\right)\right|\right)+\mathbf{1}_{G_{\delta}^{c}}+\varepsilon^{2} \\
& =\max \left(\left|B\left(\eta_{-}\right)-B(s)\right|,\left|B(t)-B\left(\eta_{+}\right)\right|\right)+\mathbf{1}_{G_{\delta}^{c}}+\varepsilon^{2} \leqslant \omega(\delta)+\mathbf{1}_{G_{\delta}^{c}}+\varepsilon^{2} .
\end{aligned}
$$

Combining the two cases, we see that for any $z \in \Omega_{\varepsilon}$,

$$
\boldsymbol{P}^{z}\left(\sup _{\substack{s, t \in[0, T] \\|t-s| \leqslant \delta}}\left|Y^{\varepsilon}(t)-Y^{\varepsilon}(s)\right|>a\right) \leqslant \boldsymbol{P}\left(\omega(\delta)>a-\varepsilon^{2}\right)+\boldsymbol{P}\left(G_{\delta}^{c}\right) .
$$

Since the right hand side is independent of $z$, integrating over $z$ with respect to $\mu^{\varepsilon}$ implies

$$
\lim _{\delta \rightarrow 0} \limsup _{\varepsilon \rightarrow 0} \boldsymbol{P}^{\mu^{\varepsilon}}\left(\sup _{\substack{s, t \in[0, T] \\|t-s| \leqslant \delta}}\left|Y^{\varepsilon}(t)-Y^{\varepsilon}(s)\right|>a\right)=0
$$

holds for any $a>0$. This shows tightness of $Y^{\varepsilon}$ in $C([0, T])$, finishing the proof of Lemma 3.1. 
3.2. Uniqueness for the Martingale Problem (Lemma 3.2). The proof of Lemma 3.2 relies on the existence of regular solutions to the corresponding parabolic equation. We state this result next.

Lemma 3.5. For all $f \in \mathcal{D}(A)$, there exists a solution to

$$
\partial_{t} u-A u=0, \quad u(\cdot, 0)=f, \quad \text { with } u(\cdot, t) \in \mathcal{D}(A) .
$$

Given Lemma 3.5, the proof of Lemma 3.2 is standard (see for instance [RW00, EK86]). For the readers convenience, we describe it briefly here.

Proof of Lemma 3.2. Suppose $Z, Z^{\prime}$ are two processes satisfying the martingale problem for $A$. Let $f \in \mathcal{D}(A)$ be any test function, and $u$ be the solution in $\mathcal{D}(A)$ of $\partial_{t} u-A u=0$ with initial data $f$. Then for any $z \in \Omega_{0}$, and fixed $T>0$, the processes $u\left(Z_{t}, T-t\right)$ and $u\left(Z_{t}^{\prime}, T-t\right)$ are both martingales under the measure $P^{z}$. Hence

$$
\begin{aligned}
\boldsymbol{E}^{\mu} f\left(Z_{T}\right) & =\int_{\Omega_{0}} \boldsymbol{E}^{z} f\left(Z_{T}\right) \mu(d z)=\int_{\Omega_{0}} \boldsymbol{E}^{z} u\left(Z_{t}, T-t\right) \mu(d z)=\int_{\Omega_{0}} u(z, T) \mu(d z) \\
& =\int_{\Omega_{0}} \boldsymbol{E}^{z} u\left(Z_{t}^{\prime}, T-t\right) \mu(d z)=\int_{\Omega_{0}} \boldsymbol{E}^{z} f\left(Z_{T}^{\prime}\right) \mu(d z)=\boldsymbol{E}^{\mu} f\left(Z_{T}^{\prime}\right) .
\end{aligned}
$$

Since $\mathcal{D}(A)$ is dense in $C_{0}\left(\Omega_{0}\right)$ this implies $Z$ and $Z^{\prime}$ have the same one dimensional distributions. By the Markov property, this in turn implies that the laws of $Z$ and $Z^{\prime}$ are the same.

It remains to prove Lemma 3.5.

Proof of Lemma 3.5. Let $v(x, t)=u(x, 0, t)$. Since (3.4) is equivalent to (1.7a)(1.7c), Proposition $1.4^{1}$ implies that $v$ satisfies the Basset type equation (1.8). For the homogeneous equation associated with (1.8), existence and uniqueness is proved in [Che17]. The inhomogeneous equation can be solved using an analog of Duhamel's principle [Uma12, US06]. Explicitly, for $s \geqslant 0$, let $\tilde{v}_{s}$ be a solution to the equation

$$
\begin{gathered}
\partial_{t} \tilde{v}_{s}(x, t)+\frac{\alpha}{2} \partial_{t}^{w} \tilde{v}_{s}(x, t)-\frac{1}{2} \partial_{x}^{2} \tilde{v}_{s}(x, t)=0, \quad \text { for } t>s, \\
\tilde{v}_{s}(x, s)=\left(I+\frac{\alpha}{2} \mathcal{I}_{s}^{w}\right)^{-1} \frac{\alpha f(x, \cdot)}{2} .
\end{gathered}
$$

Here $\mathcal{I}^{w}$ is the integral operator with kernel $w$ defined by

$$
\mathcal{I}_{t}^{w} h=\int_{0}^{t} w(t-s) h(s) d s,
$$

for any function $h:(0, \infty) \rightarrow \mathbb{R}$. Since $\mathcal{I}^{w}$ is a compact operator, the operator $\left(I+(\alpha / 2) I^{w}\right)$ is invertible, ensuring the initial condition $(3.5 \mathrm{~b})$ can be satisfied. For convenience, define $\tilde{v}_{s}(x, r)=\tilde{v}_{s}(x, s)$ when $r<s$. Now, one can directly check that the function $v$ defined by

$$
v(x, t) \stackrel{\text { def }}{=} \int_{0}^{t} \tilde{v}_{s}(x, t) d s
$$

is a strong solution to the inhomogeneous equation (1.8).

${ }^{1}$ We remark that the proof of Proposition 1.4 is self contained, and does not rely on Theorem 1.1. Thus its use here is valid and does not lead to circular logic loop. 
Since $u$ satisfies the heat equation for $y \in(0,1)$ we can write $u$ in terms of $v$ and $f$ using the heat kernel. Explicitly, we have

$$
u(x, y, t)=\frac{\alpha}{2} \int_{0}^{1} K_{t}^{\prime \prime}(y, z) f(z) d z+\kappa \int_{0}^{t} \partial_{z} K_{t-s}^{\prime \prime}(y, 0) v(x, s) d s,
$$

where $K^{\prime \prime}$ is the heat kernel on $(0,1)$ with Dirichlet boundary conditions at $y=0$ and Neumann boundary conditions at $y=1$. Since $v$ is $C^{2,1}$ this immediately implies $u \in C^{2,1}$. Thus to show $u(\cdot, t) \in \mathcal{D}(A)$ we only need to verify the flux condition (2.10). This, however, follows immediately from the fact that $\partial_{y}^{2} u(x, 0, t)=$ $2 \partial_{t} u(x, 0, t)=2 \partial_{t} v(x, t)$ and equation $(2.22)$.

3.3. Generator Estimate (Lemma 3.3). The main idea behind the proof of Lemma 3.3 is to balance the local time $Z^{\varepsilon}$ spends at the "gate" between the spine and teeth, and the time spent in the spine. Explicitly, let $S \stackrel{\text { def }}{=} \mathbb{R} \times(-\varepsilon, 0)$ denote the spine of $\Omega_{\varepsilon}$, and $T$, defined by

$$
T \stackrel{\text { def }}{=} \bigcup_{k \in \varepsilon \mathbb{Z}}\left\{(x, y)|| x-\varepsilon k \mid<\frac{\alpha \varepsilon^{2}}{2}, y \in(0,1)\right\},
$$

denote the collection of the teeth (see (1.1) and Figure 1). Let the "gate" $G$, defined by

$$
G \stackrel{\text { def }}{=} \partial T \cap \partial S=\bigcup_{k \in \varepsilon \mathbb{Z}}\left\{(x, 0)|| x-\varepsilon k \mid \leqslant \frac{\alpha \varepsilon^{2}}{2}\right\},
$$

denote the union of short segments connecting the spine and teeth. Let $L_{t}^{G}$ denote the local time of $Z_{t}^{\varepsilon}$ at the set $G$. Now the required local time balance can be stated as follows.

Lemma 3.6. For every $g \in C_{b}^{1}(\mathbb{R})$ and $K \subseteq \Omega_{0}$ compact we have

$$
\lim _{\varepsilon \rightarrow 0} \sup _{z \in K \cap \Omega_{\varepsilon}} \boldsymbol{E}^{z}\left(\alpha \int_{0}^{t} g\left(X_{s}^{\varepsilon}\right) \mathbf{1}_{\left\{Y_{s}^{\varepsilon}<0\right\}} d s-2 \int_{0}^{t} g\left(X_{s}^{\varepsilon}\right) d L_{s}^{G}\right)=0 .
$$

Next, we will also need to show that the local times on the left edges and right edges of the teeth balance. Explicitly, let $\partial T^{-}, \partial T^{+}$defined by

$$
\begin{aligned}
\partial T^{-} \stackrel{\text { def }}{=}\left\{(x, y) \in Z^{\varepsilon} \mid x \in \varepsilon \mathbb{Z}-\frac{\alpha \varepsilon^{2}}{2}, y>0\right\}, \\
\text { and } \quad \partial T^{+} \stackrel{\text { def }}{=}\left\{(x, y) \in Z^{\varepsilon} \mid x \in \varepsilon \mathbb{Z}+\frac{\alpha \varepsilon^{2}}{2}, y>0\right\} .
\end{aligned}
$$

denote the left and right edges of the teeth respectively. Let $L^{+}$and $L^{-}$be the local times of $Z^{\varepsilon}$ about $\partial T^{-}$and $\partial T^{+}$respectively, and let $L^{ \pm}$denote the difference

$$
L^{ \pm}=L^{-}-L^{+} .
$$

The balance on the teeth boundaries we require is as follows.

Lemma 3.7. For every $f \in \mathcal{D}(A)$ and $K \subseteq \Omega_{0}$ compact, we have

$$
\lim _{\varepsilon \rightarrow 0} \sup _{z \in K \cap \Omega_{\varepsilon}} \boldsymbol{E}^{z}\left(\int_{0}^{t} \frac{1}{2} \partial_{x}^{2} f\left(Z_{s}^{\varepsilon,+}\right) \mathbf{1}_{\left\{Y_{s}^{\varepsilon}>0\right\}} d s+\int_{0}^{t} \partial_{x} f\left(Z_{s}^{\varepsilon,+}\right) d L_{s}^{ \pm}\right)=0 .
$$

Momentarily postponing the proofs of Lemmas 3.6 and 3.7, we prove Lemma 3.3. 
Proof of Lemma 3.3. Given $f \in \mathcal{D}(A)$, we define $f^{\varepsilon}: \Omega_{\varepsilon} \rightarrow \mathbb{R}$ by

$$
f^{\varepsilon}(x, y) \stackrel{\text { def }}{=} f\left(x, y^{+}\right) .
$$

Thus, $f\left(Z_{t}^{\varepsilon,+}\right)=f^{\varepsilon}\left(Z_{t}^{\varepsilon}\right)$, and (3.1) reduces to showing

$$
\lim _{\varepsilon \rightarrow 0} \sup _{z \in K \cap \Omega_{\varepsilon}} \boldsymbol{E}^{z}\left(f^{\varepsilon}\left(Z_{t}^{\varepsilon}\right)-f^{\varepsilon}\left(Z_{0}^{\varepsilon}\right)-\int_{0}^{t} \frac{1}{2} \partial_{y}^{2} f^{\varepsilon}\left(Z_{s}^{\varepsilon}\right) d s\right)=0 .
$$

Since $f \in \mathcal{D}(A)$, we have $\partial_{x}^{2} f(x, 0)+\alpha \partial_{y} f(x, 0)=\partial_{y}^{2} f(x, 0)$ and $\partial_{y} f(x, 1)=0$. Therefore, the extension $f^{\varepsilon}$ satisfies $\partial_{x}^{2} f^{\varepsilon}(x, y)=\partial_{y}^{2} f^{\varepsilon}\left(x, 0^{+}\right)-\alpha \partial_{y} f^{\varepsilon}\left(x, 0^{+}\right)$for $(x, y) \in S$, as well as $\partial_{y} f^{\varepsilon}=0$ for $(x, y) \in S$. Notice that $\partial_{y} f^{\varepsilon}$ may be discontinuous across $G$. Using these facts and Itô's formula, we compute

$$
\begin{aligned}
\boldsymbol{E}^{z}\left(f^{\varepsilon}\left(Z_{t}^{\varepsilon}\right)-f^{\varepsilon}\left(Z_{s}^{\varepsilon}\right)\right)= & \boldsymbol{E}^{z}\left(\int_{0}^{t} \frac{1}{2}\left(\partial_{y}^{2} f\left(Z_{s}^{\varepsilon,+}\right)+\partial_{x}^{2} f\left(Z_{s}^{\varepsilon,+}\right)\right) \mathbf{1}_{\left\{Y_{s}^{\varepsilon}>0\right\}} d s\right) \\
& +\boldsymbol{E}^{z}\left(\int_{0}^{t} \frac{1}{2} \partial_{x}^{2} f\left(X_{s}^{\varepsilon}, 0^{+}\right) \mathbf{1}_{\left\{Y_{s}^{\varepsilon}<0\right\}} d s\right) \\
& +\boldsymbol{E}^{z}\left(\int_{0}^{t} \partial_{y} f\left(X_{s}^{\varepsilon}, 0^{+}\right) d L_{s}^{G}+\int_{0}^{t} \partial_{x} f\left(Z_{s}^{\varepsilon,+}\right) d L_{s}^{ \pm}\right) \\
= & \boldsymbol{E}^{z}\left(\int_{0}^{t} \frac{1}{2}\left(\partial_{y}^{2} f\left(Z_{s}^{\varepsilon,+}\right)+\partial_{x}^{2} f\left(Z_{s}^{\varepsilon,+}\right)\right) \mathbf{1}_{\left\{Y_{s}^{\varepsilon}>0\right\}} d s\right) \\
& +\boldsymbol{E}^{z}\left(\frac{1}{2} \int_{0}^{t}\left(\partial_{y}^{2} f\left(X_{s}^{\varepsilon}, 0^{+}\right)-\alpha \partial_{y} f\left(X_{s}^{\varepsilon}, 0^{+}\right)\right) \mathbf{1}_{\left\{Y_{s}^{\varepsilon}<0\right\}} d s\right) \\
& +\boldsymbol{E}^{z}\left(\int_{0}^{t} \partial_{y} f\left(X_{s}^{\varepsilon}, 0^{+}\right) d L_{s}^{G}+\int_{0}^{t} \partial_{x} f\left(Z_{s}^{\varepsilon,+}\right) d L_{s}^{ \pm}\right),
\end{aligned}
$$

and hence

$$
\begin{aligned}
\boldsymbol{E}^{z}\left(f^{\varepsilon}\left(Z_{t}^{\varepsilon}\right)\right. & \left.-f^{\varepsilon}\left(Z_{0}^{\varepsilon}\right)-\int_{0}^{t} \frac{1}{2} \partial_{y}^{2} f^{\varepsilon}\left(Z_{s}^{\varepsilon}\right) d s\right) \\
= & \boldsymbol{E}^{z}\left(\int_{0}^{t} \frac{1}{2} \partial_{x}^{2} f\left(Z_{s}^{\varepsilon,+}\right) \mathbf{1}_{\left\{Y_{s}^{\varepsilon}>0\right\}} d s+\int_{0}^{t} \partial_{x} f\left(Z_{s}^{\varepsilon,+}\right) d L_{s}^{ \pm}\right) \\
& -\frac{1}{2} \boldsymbol{E}^{z}\left(\int_{0}^{t} \alpha \partial_{y} f\left(X_{s}^{\varepsilon}, 0^{+}\right) \mathbf{1}_{\left\{Y_{s}^{\varepsilon}<0\right\}} d s-2 \int_{0}^{t} \partial_{y} f\left(X_{s}^{\varepsilon}, 0^{+}\right) d L_{s}^{G}\right) .
\end{aligned}
$$

Using Lemmas 3.6 and 3.7 we see that the supremum over $z \in \Omega_{\varepsilon} \cap K$ of the right hand side of the above vanishes as $\varepsilon \rightarrow 0$. This proves Lemma 3.3.

It remains to prove Lemmas 3.6 and 3.7, and we do this in Sections 3.4 and 3.6 respectively.

3.4. Local Time at the Gate (Lemma 3.6). The crux in the proof of Lemma 3.6 is an oscillation estimate on the solution to a specific Poisson equation with Neumann boundary conditions (Proposition 3.8, below). We state this when it is first encountered, and prove it in the next subsection.

Proof of Lemma 3.6. The expectation in (3.6) can be written as

$$
\boldsymbol{E}^{z}\left(\int_{0}^{t} \alpha g\left(X_{s}^{\varepsilon}\right) \mathbf{1}_{\left\{Y_{s}^{\varepsilon}<0\right\}} d s-2 \int_{0}^{t} g\left(X_{s}^{\varepsilon}\right) d L_{s}^{G}\right)
$$




$$
\begin{array}{r}
=\sum_{k \in \mathbb{Z}} g(\varepsilon k) \boldsymbol{E}^{z}\left(\alpha \int_{0}^{t} \mathbf{1}_{\left\{Y_{s}^{\varepsilon}<0\right\}} \mathbf{1}_{\left\{\left|X_{s}^{\varepsilon}-\varepsilon k\right|<\varepsilon / 2\right\}} d s-2 \int_{0}^{t} \mathbf{1}_{\left\{\left|X_{s}^{\varepsilon}-\varepsilon k\right|<\varepsilon / 2\right\}} d L_{s}^{G}\right) \\
+R^{\varepsilon}
\end{array}
$$

where the remainder term $R^{\varepsilon}$ is given by

$$
\begin{aligned}
R^{\varepsilon} \stackrel{\text { def }}{=} \alpha \sum_{k \in \mathbb{Z}} \boldsymbol{E}^{z}\left(\int_{0}^{t}\left(g\left(X_{s}^{\varepsilon}\right)-g(\varepsilon k)\right) \mathbf{1}_{\left\{Y_{s}^{\varepsilon}<0\right\}} \mathbf{1}_{\left\{\left|X_{s}^{\varepsilon}-\varepsilon k\right|<\varepsilon / 2\right\}} d s\right) \\
\quad-2 \boldsymbol{E}^{z}\left(\int_{0}^{t}\left(g\left(X_{s}^{\varepsilon}\right)-g(\varepsilon k)\right) \mathbf{1}_{\left\{\left|X_{s}^{\varepsilon}-\varepsilon k\right|<\varepsilon / 2\right\}} d L_{s}^{G}\right) \stackrel{\text { def }}{=} R_{1}^{\varepsilon}+R_{2}^{\varepsilon} .
\end{aligned}
$$

To estimate $R^{\varepsilon}$, for any $\delta>0$ we choose sufficiently large $M>0$ such that

$$
\sup _{(x, y) \in K} \boldsymbol{E}\left(\int_{0}^{t} \mathbf{1}_{\left\{|x|+4\left|W_{s}\right|+2 \geqslant M\right\}} d s\right)<\frac{\delta}{\|g\|_{\infty}},
$$

where $W$ is a standard Brownian motion in $\mathbb{R}$. Here we write $\boldsymbol{P}$ and $\boldsymbol{E}$ (without superscripts) to denote the probability measure and expected value for a standard Brownian motion. By Lemma 3.4, we have

$$
\boldsymbol{P}^{z}\left(\left|X_{s}^{\varepsilon}\right|+1 \geqslant M\right) \leqslant \boldsymbol{P}\left(x+4\left|W_{s}\right|+2 \geqslant M\right),
$$

where $z=(x, y)$ and so the above estimate can be applied for $X^{\varepsilon}$ independent of $\varepsilon \in(0,1 / 2]$. Since $g$ is continuous and hence uniformly continuous on $[-M, M]$, for any $\delta>0$ we can choose $\varepsilon>0$ such that if $x_{1}, x_{2} \in[-M, M]$ with $\left|x_{1}-x_{2}\right|<\varepsilon$ then $\left|g\left(x_{1}\right)-g\left(x_{2}\right)\right|<\delta$. For such $\varepsilon$ and for integers $k \in \varepsilon^{-1}[-M, M]$ we have

$$
\begin{aligned}
\boldsymbol{E}^{(x, y)} \int_{0}^{t}\left|g(\varepsilon k)-g\left(X_{s}^{\varepsilon}\right)\right| \mathbf{1}_{\left\{Y_{s}^{\varepsilon}<0,\left|X_{s}^{\varepsilon}-\varepsilon k\right|<\varepsilon / 2\right\}} d s & \\
& \leqslant \delta \int_{0}^{t} \boldsymbol{P}^{z}\left(\left|X_{s}^{\varepsilon}-\varepsilon k\right|<\varepsilon / 2\right) d s .
\end{aligned}
$$

Combining the above with (3.9), gives the following estimate of $R_{1}^{\varepsilon}$

$$
\begin{aligned}
& \left|R_{1}^{\varepsilon}\right| \leqslant \alpha\left(\delta \sum_{\substack{k \in \mathbb{Z} \\
\varepsilon k \in[-M, M]}} \int_{0}^{t} \boldsymbol{P}^{z}\left(\left|X_{s}^{\varepsilon}-\varepsilon k\right|<\frac{\varepsilon}{2}\right) d s\right. \\
& \left.\quad+2\|g\|_{\infty} \sum_{|\varepsilon k|>M} \int_{0}^{t} \boldsymbol{P}^{z}\left(\left|X_{s}^{\varepsilon}-\varepsilon k\right|<\frac{\varepsilon}{2}\right) d s\right) \\
& \leqslant \alpha(t+2) \delta .
\end{aligned}
$$

Since $\delta>0$ was arbitrary this proves $R_{1}^{\varepsilon} \rightarrow 0$ as $\varepsilon \rightarrow 0$. An estimate for $R_{2}^{\varepsilon}$ can be obtained in the same manner. Namely,

$$
\begin{aligned}
\left|R_{2}^{\varepsilon}\right| & \leqslant 2\left(\delta \boldsymbol{E}^{z}\left(L_{t}^{G}\right)+2\|g\|_{\infty} \sum_{\substack{k \in \mathbb{Z} \\
|\varepsilon k| \geqslant M}} \boldsymbol{E}^{z}\left(\int_{0}^{t} \mathbf{1}_{\left\{\left|X_{s}^{\varepsilon}-\varepsilon k\right|<\varepsilon / 2\right\}} d L_{s}^{G}\right)\right) \\
& \leqslant c(t) \delta+2\|g\|_{\infty} \boldsymbol{E}^{x}\left(\int_{0}^{t} \mathbf{1}_{\left\{\left|X_{s}^{\varepsilon}\right|+1 \geqslant M\right\}} d L_{s}^{G}\right) .
\end{aligned}
$$


Let $\tau=\inf \left\{t|| X_{t}^{\varepsilon} \mid+1 \geqslant M\right\}$ and note that by the Markov property

$$
\begin{aligned}
\boldsymbol{E}^{z}\left(\int_{0}^{t} \mathbf{1}_{\left\{\mid X_{s}^{\varepsilon \mid+1 \geqslant M\}}\right.} d L_{s}^{G}\right) & \leqslant \boldsymbol{E}^{z}\left(\boldsymbol{E}^{X_{\tau}^{\varepsilon}}\left(L_{t-t \wedge \tau}^{G}\right)\right) \\
& \leqslant\left(\sup _{z^{\prime}} \boldsymbol{E}^{z^{\prime}}\left(L_{t}^{G}\right)\right) \boldsymbol{P}^{z}(\tau<t) .
\end{aligned}
$$

Applying Itô's formula to $w\left(Z^{\varepsilon}\right)$, where

$$
w(x, y) \stackrel{\text { def }}{=} \begin{cases}\frac{1}{2}(1-y)^{2}, & y \in[0,1], \\ 0, & \text { otherwise }\end{cases}
$$

shows

$$
\boldsymbol{E}^{z}\left(L_{t}^{G}\right)=O(1) \quad \text { as } t \rightarrow 0 .
$$

By choosing $M$ larger, if necessary, we have

$$
\sup _{z \in K} \boldsymbol{P}^{z}(\tau<t)<\delta
$$

for all $\varepsilon \in(0,1 / 2]$. Since $\delta>0$ is arbitrary, this shows that $R_{2}^{\varepsilon} \rightarrow 0$ as $\varepsilon \rightarrow 0$.

Next, we need a PDE estimate to control the expression

$$
\boldsymbol{E}^{z}\left(\alpha \int_{0}^{t} \mathbf{1}_{\left\{Y_{s}^{\varepsilon}<0\right\}} \mathbf{1}_{\left\{\left|X_{s}-\varepsilon k\right|<\varepsilon / 2\right\}} d s-2 \int_{0}^{t} \mathbf{1}_{\left\{\left|X_{s}-\varepsilon k\right|<\varepsilon / 2\right\}} d L_{s}^{G}\right) .
$$

from (3.8). To this end, let $Q$ be a region of width $\varepsilon$ directly below the tooth at $x=0$, and $G_{0}$ be the component of $G$ contained in $[-\varepsilon / 2, \varepsilon / 2] \times \mathbb{R}$. Explicitly, let

$$
Q \stackrel{\text { def }}{=}\left[-\frac{\varepsilon}{2}, \frac{\varepsilon}{2}\right] \times[-\varepsilon, 0] \quad \text { and } \quad G_{0}=\left\{(x, 0) \mid-\frac{\alpha \varepsilon^{2}}{2}<x<\frac{\alpha \varepsilon^{2}}{2}\right\} .
$$

Let $\mu^{\varepsilon}$ denote the one dimensional Hausdorff measure supported on $G_{0}$ (i.e. a measure supported on $G_{0}$ ).

Proposition 3.8. Let the function $u^{\varepsilon}: \Omega_{\varepsilon} \rightarrow \mathbb{R}$ be the solution of

$$
\begin{array}{cc}
-\Delta u^{\varepsilon}=\alpha \mathbf{1}_{Q}-\mu^{\varepsilon} & \text { in } \Omega_{\varepsilon} \\
\partial_{\nu} u^{\varepsilon}=0 & \text { on } \partial \Omega_{\varepsilon},
\end{array}
$$

with the normalization condition

$$
\inf _{\Omega_{\varepsilon}} u^{\varepsilon}=0 .
$$

Then there exists a constant $C>0$, independent of $\varepsilon$ such that

$$
\sup _{\Omega_{\varepsilon}} u^{\varepsilon}(z) \leqslant C \varepsilon^{2}|\ln \varepsilon| .
$$

Remark. Existence of a solution to (3.13)-(3.14) can be proved by using [Dro00, Thm. 2.2] and a standard approximation argument to deal with the unbounded domain. See also [Gri85, Thm. 2.2.1.3].

Throughout the remainder of this proof and the section, we will use the convention that $C>0$ is a constant that is independent of $\varepsilon$. We apply Itô's formula to the function $u^{\varepsilon}$ defined in Proposition 3.8 to obtain

$$
2 \boldsymbol{E}^{z}\left(u^{\varepsilon}\left(Z_{t}^{\varepsilon}\right)-u^{\varepsilon}\left(Z_{0}^{\varepsilon}\right)\right)=-\boldsymbol{E}^{z}\left(\alpha \int_{0}^{t} \mathbf{1}_{Q}\left(Z_{s}^{\varepsilon}\right) d s-2 L_{t}^{G_{0}}\right) .
$$




$$
=\boldsymbol{E}^{z}\left(\alpha \int_{0}^{t} \mathbf{1}_{\left\{Y_{s}^{\varepsilon}<0\right\}} \mathbf{1}_{\left\{\left|X_{s}\right|<\varepsilon / 2\right\}} d s-2 \int_{0}^{t} \mathbf{1}_{\left\{\left|X_{s}\right|<\varepsilon / 2\right\}} d L_{s}^{G}\right) .
$$

The oscillation bound (3.16) now implies

$$
\left|\boldsymbol{E}^{z}\left(\alpha \int_{0}^{t} \mathbf{1}_{\left\{Y_{s}^{\varepsilon}<0\right\}} \mathbf{1}_{\left\{\left|X_{s}-\varepsilon k\right|<\varepsilon / 2\right\}} d s-2 \int_{0}^{t} \mathbf{1}_{\left\{\left|X_{s}-\varepsilon k\right|<\varepsilon / 2\right\}} d L_{s}^{G}\right)\right| \leqslant C \varepsilon^{2}|\log \varepsilon|
$$

holds for all $k$ and $x \in \mathbb{R}$. Because of (3.9), we can restrict the sum in (3.8) to $k \in \mathbb{Z}$ for which $\varepsilon|k| \leqslant M$ (i.e. only $O\left(\varepsilon^{-1}\right)$ terms in the sum). Therefore,

$$
\begin{array}{r}
\sum_{\substack{k \in \mathbb{Z} \\
\varepsilon|k| \leqslant M}} \boldsymbol{E}^{z}\left(\alpha \int_{0}^{t} \mathbf{1}_{\left\{Y_{s}^{\varepsilon}<0\right\}} \mathbf{1}_{\left\{\left|X_{s}^{\varepsilon}-\varepsilon k\right|<\varepsilon / 2\right\}} d s-2 \int_{0}^{t} \mathbf{1}_{\left\{\left|X_{s}^{\varepsilon}-\varepsilon k\right|<\varepsilon / 2\right\}} d L_{s}^{G}\right) \\
\leqslant O(\varepsilon|\log (\varepsilon)|) .
\end{array}
$$

Combining this with the above estimates, we conclude that (3.6) holds.

To complete the proof of Lemma 3.6, it remains to prove Proposition 3.8. We do this in the next subsection.

3.5. An Oscillation Estimate for the Neumann Problem (Proposition 3.8). The proof of Proposition 3.8 involves a "geometric series" argument using the probabilistic representation. Explicitly, we obtain the desired oscillation estimate by estimating the probabilities of successive visits of $Z^{\varepsilon}$ between two segments. The key step in the proof involves the so called narrow escape problem (see for instance [HS14]), which guarantees that the probability that Brownian motion exists from a given interval on the boundary of a domain vanishes logarithmically with the interval size. In our specific scenario, however, we can not directly use the results of [HS14] and we prove the required estimates here.

Proof of Proposition 3.8. Note first that

$$
\int_{\Omega_{\varepsilon}}\left(\alpha \mathbf{1}_{Q}-\mu^{\varepsilon}\right) d z=0
$$

and hence a bounded solution to (3.13)-(3.14) exists. Moreover, because the measure $\alpha \mathbf{1}_{Q}(z)-\mu^{\varepsilon}$ is supported in $\bar{Q}$, the function $u^{\varepsilon}$ is harmonic in $\Omega_{\varepsilon}-\bar{Q}$. Thus, by the maximum principle,

$$
\sup _{\Omega_{\varepsilon}} u^{\varepsilon} \leqslant \sup _{Q} u^{\varepsilon}
$$

Define $Q^{\prime} \supseteq Q$ to be the region that enlarges $Q$ by $\varepsilon^{2}$ on the top, and $\varepsilon / 4$ on the sides. Precisely, let

$$
Q^{\prime} \stackrel{\text { def }}{=} \Omega_{\varepsilon} \bigcap\left(\left[-\frac{3 \varepsilon}{4}, \frac{3 \varepsilon}{4}\right] \times\left[-\varepsilon, \varepsilon^{2}\right]\right) .
$$

The first step is to estimate the oscillation of $u^{\varepsilon}$ on the top and side portion of $Q^{\prime}$. Let $A^{\prime}$ and $D^{\prime}$, defined by

$$
A^{\prime} \stackrel{\text { def }}{=}\left[-\frac{\alpha \varepsilon^{2}}{2}, \frac{\alpha \varepsilon^{2}}{2}\right] \times\left\{\alpha \varepsilon^{2}\right\} \quad \text { and } \quad D^{\prime} \stackrel{\text { def }}{=}\left\{ \pm \frac{3 \varepsilon}{4}\right\} \times[-\varepsilon, 0]
$$

denotes the top and sides of $Q^{\prime}$ respectively. We aim to show

$$
\sup _{a, d \in A^{\prime} \cup D^{\prime}}\left|u^{\varepsilon}(a)-u^{\varepsilon}(d)\right| \leqslant C \varepsilon^{2}|\ln \varepsilon| .
$$




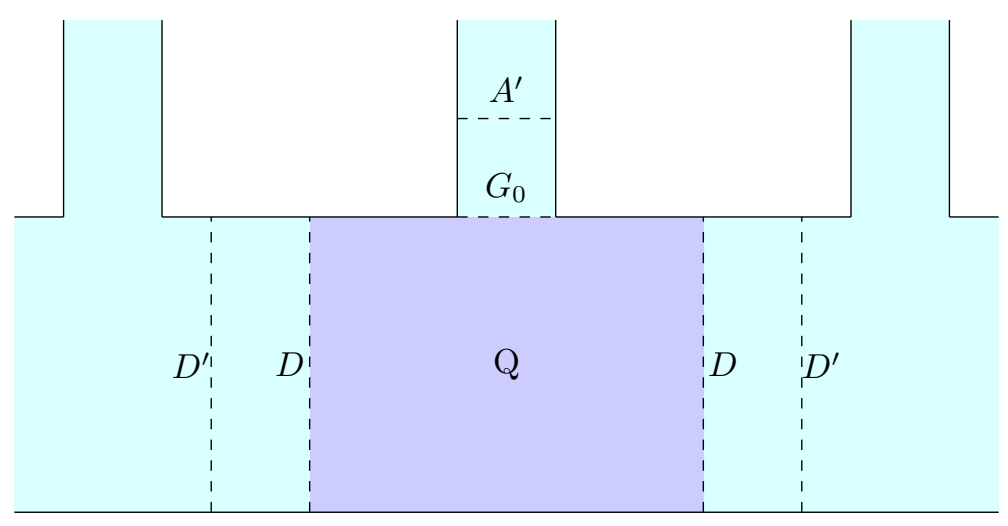

FIGURE 3 . Image of one period of $\Omega_{\varepsilon}$.

Let $\tau_{0}$ be the first time at which the process $Z_{t}^{\varepsilon}$ hits the gate $G_{0}$ (defined in (3.12)). The stopping time $\tau_{0}$ is finite almost surely, but has infinite expectation. We claim that the distribution of $Z_{\tau_{0}}^{\varepsilon}$ on $G$ is bounded below by a constant multiple of the Hausdorff measure, uniformly over all initial points in $A^{\prime} \cup D^{\prime}$.

Lemma 3.9. For any $z \in A^{\prime} \cup D^{\prime}$, let $\rho(z, \cdot)$, defined by

$$
\rho(z, r)=\boldsymbol{P}^{z}\left(Z_{\tau_{0}}^{\varepsilon} \in d r\right),
$$

denote the density of the random variable $Z_{\tau_{0}}^{\varepsilon}$ on $G_{0}$. Then, there exists $\delta>0$ such that

$$
\rho(z, r) \geqslant \frac{\delta}{\alpha \varepsilon^{2}}
$$

for all $z \in A^{\prime} \cup D^{\prime}$ and $r \in G_{0}$.

Momentarily postponing the proof of this lemma, we note that for any $a, d \in$ $A^{\prime} \cup D^{\prime}$, we have

$$
\begin{gathered}
\boldsymbol{E}^{a} u^{\varepsilon}\left(Z_{\tau_{0}}^{\varepsilon}\right)-\boldsymbol{E}^{d} u^{\varepsilon}\left(Z_{\tau_{0}}^{\varepsilon}\right)=\int_{G_{0}} \rho(a, r) u^{\varepsilon}(r) d r-\int_{G_{0}} \rho(d, r) u^{\varepsilon}(r) d r \\
\quad=\int_{G_{0}}\left(\rho(a, r)-\frac{\delta}{\alpha \varepsilon^{2}}\right) u^{\varepsilon}(r) d r-\int_{G_{0}}\left(\rho(d, r)-\frac{\delta}{\alpha \varepsilon^{2}}\right) u^{\varepsilon}(r) d r \\
\leqslant(1-\delta)\left(\sup _{G_{0}} u^{\varepsilon}-\inf _{G_{0}} u^{\varepsilon}\right) \leqslant(1-\delta)\left(\sup _{r_{1}, r_{2} \in G_{0}}\left|u^{\varepsilon}\left(r_{1}\right)-u^{\varepsilon}\left(r_{2}\right)\right|\right) .
\end{gathered}
$$

To obtain the second last inequality above we used the fact that

$$
\rho(z, r)-\frac{\delta}{\alpha \varepsilon^{2}} \geqslant 0
$$

which is guaranteed by Lemma 3.9.

Now by Itô's formula,

$$
\begin{aligned}
u^{\varepsilon}(a)-u^{\varepsilon}(d)= & \boldsymbol{E}^{a} u^{\varepsilon}\left(Z_{\tau_{0}}^{\varepsilon}\right)-\boldsymbol{E}^{d} u^{\varepsilon}\left(Z_{\tau_{0}}^{\varepsilon}\right)-\frac{1}{2} \boldsymbol{E}^{a}\left(2 L_{\tau_{0}}^{G_{0}^{+}}-\alpha \int_{0}^{\tau_{0}} \mathbf{1}_{Q}\left(Z_{s}^{\varepsilon}\right) d s\right) \\
+ & \frac{1}{2} \boldsymbol{E}^{d}\left(2 L_{\tau_{0}}^{G_{0}^{+}}-\alpha \int_{0}^{\tau_{0}} \mathbf{1}_{Q}\left(Z_{s}^{\varepsilon}\right) d s\right)
\end{aligned}
$$




$$
\begin{gathered}
\leqslant(1-\delta) \sup _{r_{1}, r_{2} \in G_{0}}\left|u^{\varepsilon}\left(r_{1}\right)-u^{\varepsilon}\left(r_{2}\right)\right|-\frac{1}{2} \boldsymbol{E}^{a}\left(2 L_{\tau_{0}}^{G_{0}^{+}}-\alpha \int_{0}^{\tau_{0}} \mathbf{1}_{Q}\left(Z_{s}^{\varepsilon}\right) d s\right) \\
+\frac{1}{2} \boldsymbol{E}^{d}\left(2 L_{\tau_{0}}^{G_{0}^{+}}-\alpha \int_{0}^{\tau_{0}} \mathbf{1}_{Q}\left(Z_{s}^{\varepsilon}\right) d s\right)
\end{gathered}
$$

Note that by definition of $\tau_{0}$ we have we have $L_{\tau_{0}}^{G_{0}^{+}}=0$ for all $a, d \in A^{\prime} \cup D^{\prime}$. Also, if $a \in A^{\prime}$, then $Y_{s}^{\varepsilon}>0$ for all $s \in\left[0, \tau_{0}\right]$ with probability one. Hence

$$
\begin{aligned}
\sup _{a, d \in A^{\prime} \cup D^{\prime}} \mid & \mid \frac{1}{2} \boldsymbol{E}^{a}\left(2 L_{\tau_{0}}^{G_{0}^{+}}-\alpha \int_{0}^{\tau_{0}} \mathbf{1}_{Q}\left(Z_{s}^{\varepsilon}\right) d s\right) \\
+ & \frac{1}{2} \boldsymbol{E}^{d}\left(2 L_{\tau_{0}}^{G_{0}^{+}}-\alpha \int_{0}^{\tau_{0}} \mathbf{1}_{Q}\left(Z_{s}^{\varepsilon}\right) d s\right) \mid \leqslant \alpha \sup _{d \in D^{\prime}} \boldsymbol{E}^{d} \int_{0}^{\tau_{0}} \mathbf{1}_{Q}\left(Z_{s}^{\varepsilon}\right) d s .
\end{aligned}
$$

We claim that the term on the right is bounded by $C \varepsilon^{2}|\ln \varepsilon|$. To avoid distracting from the main proof, we single this out as a lemma and postpone the proof.

Lemma 3.10. With the above notation,

$$
\sup _{d \in D^{\prime}} \boldsymbol{E}^{d} \int_{0}^{\tau_{0}} \mathbf{1}_{Q}\left(Z_{s}^{\varepsilon}\right) d s \leqslant C \varepsilon^{2}|\ln \varepsilon| .
$$

Using Lemma 3.10 and (3.22) in (3.21) we conclude

$$
\sup _{a, d \in A^{\prime} \cup D^{\prime}}\left|u^{\varepsilon}(a)-u^{\varepsilon}(d)\right| \leqslant(1-\delta) \sup _{r_{1}, r_{2} \in G_{0}}\left|u^{\varepsilon}\left(r_{1}\right)-u^{\varepsilon}\left(r_{2}\right)\right|+C \varepsilon^{2}|\ln \varepsilon| .
$$

To finish proving (3.18), we will now have to control the oscillation of $u^{\varepsilon}$ on $G_{0}$ in terms of the oscillation of $u^{\varepsilon}$ on $A^{\prime} \cup D^{\prime}$.

For this, given $Z_{0}^{\varepsilon} \in G_{0}$, let $\tau_{0}^{\prime}$ be the first time that $Z_{t}^{\varepsilon}$ hits $A^{\prime} \cup D^{\prime}$. By Itô's formula again, we have for all $r_{1}, r_{2} \in G_{0}$ :

$$
\begin{aligned}
u^{\varepsilon}\left(r_{1}\right)-u^{\varepsilon}\left(r_{2}\right) \leqslant & \sup _{a^{\prime}, d^{\prime} \in A^{\prime} \cup D^{\prime}}\left(u^{\varepsilon}\left(a^{\prime}\right)-u^{\varepsilon}\left(d^{\prime}\right)\right) \\
& -\frac{1}{2} \boldsymbol{E}^{r_{1}}\left(2 L_{\tau_{0}^{\prime}}^{G_{0}}-\alpha \int_{0}^{\tau_{0}^{\prime}} \mathbf{1}_{Q} d s\right)+\frac{1}{2} \boldsymbol{E}^{r_{2}}\left(2 L_{\tau_{0}^{\prime}}^{G_{0}}-\alpha \int_{0}^{\tau_{0}^{\prime}} \mathbf{1}_{Q} d s\right) .
\end{aligned}
$$

We claim that the last two terms above are $O\left(\varepsilon^{2}\right)$. For clarity of presentation we single this out as a Lemma and postpone the proof.

Lemma 3.11. With the above notation

$$
\sup _{r \in G_{0}}\left|\boldsymbol{E}^{r}\left(2 L_{\tau_{0}^{\prime}}^{G_{0}}-\alpha \int_{0}^{\tau_{0}^{\prime}} \mathbf{1}_{Q}\left(Z_{s}^{\varepsilon}\right) d s\right)\right| \leqslant C \varepsilon^{2} .
$$

Using (3.24) and Lemma 3.11, we see

$$
\sup _{r_{1}, r_{2} \in G_{0}}\left|u^{\varepsilon}\left(r_{1}\right)-u^{\varepsilon}\left(r_{2}\right)\right| \leqslant \sup _{a, d \in A^{\prime} \cup D^{\prime}}\left|u^{\varepsilon}(a)-u^{\varepsilon}(d)\right|+C \varepsilon^{2} .
$$

Combining this with (3.23), we obtain

$$
\sup _{a, d \in A^{\prime} \cup D^{\prime}}\left|u^{\varepsilon}(a)-u^{\varepsilon}(d)\right| \leqslant(1-\delta)\left(\sup _{a, d \in A^{\prime} \cup D^{\prime}}\left|u^{\varepsilon}(a)-u^{\varepsilon}(d)\right|+C \varepsilon^{2}|\ln \varepsilon|\right)+C \varepsilon^{2} .
$$

and hence

$$
\sup _{a, d \in A^{\prime} \cup D^{\prime}}\left|u^{\varepsilon}(a)-u^{\varepsilon}(d)\right| \leqslant C\left(\frac{1-\delta}{\delta}\right) \varepsilon^{2}|\ln \varepsilon|+\frac{C}{\delta} \varepsilon^{2} .
$$

This proves (3.18) as desired. 
Now we turn this into an oscillation bound on $u^{\varepsilon}$ over the interior. Observe that for any $z \in \Omega_{\varepsilon}$,

$$
u^{\varepsilon}(z)=\boldsymbol{E}^{z}\left[u^{\varepsilon}\left(Z_{\tau_{0}^{\prime}}^{\varepsilon}\right)\right]+\frac{1}{2} \boldsymbol{E}^{z}\left(2 L_{\tau_{0}^{\prime}}^{Y^{\varepsilon}}\left(0^{+}\right)-\alpha \int_{0}^{\tau_{0}^{\prime}} \mathbf{1}_{\left\{Y_{s}^{\varepsilon} \leqslant 0\right\}} d s\right)
$$

These last terms can be estimated with the same argument used in Lemma 3.11, leading to

$$
\sup _{z \in \Omega_{\varepsilon}}\left|u^{\varepsilon}(z)-\boldsymbol{E}^{z} u^{\varepsilon}\left(Z_{\tau_{0}^{\prime}}^{\varepsilon}\right)\right| \leqslant C \varepsilon^{2} .
$$

The combination of this and (3.26) implies that

$$
\sup _{z_{1}, z_{2} \in \Omega_{\varepsilon}}\left|u^{\varepsilon}\left(z_{1}\right)-u^{\varepsilon}\left(z_{2}\right)\right| \leqslant \sup _{z_{1}, z_{2} \in \Omega_{\varepsilon}}\left|\boldsymbol{E}^{z_{1}} u^{\varepsilon}\left(Z_{\tau_{0}^{\prime}}^{\varepsilon}\right)-\boldsymbol{E}^{z_{2}} u^{\varepsilon}\left(Z_{\tau_{0}^{\prime}}^{\varepsilon}\right)\right|+C \varepsilon^{2} \leqslant C \varepsilon^{2}(|\ln \varepsilon|+1) \text {. }
$$

This implies (3.16), concluding the proof.

For the proof of Lemma 3.9 we will use a standard large deviation estimate for Brownian motion. We state the result we need below.

Lemma 3.12. Let $W_{t}$ be a standard Brownian motion in $\mathbb{R}^{d}$. Let $\gamma \in C\left([0, T] ; \mathbb{R}^{d}\right)$ be absolutely continuous with $S(\gamma)=\int_{0}^{T}\left|\gamma^{\prime}(s)\right|^{2} d s<\infty$. Then

$$
\boldsymbol{P}\left(\sup _{t \in[0, T]}|W(t)-\gamma(t)| \leqslant \delta\right) \geqslant \frac{\boldsymbol{P}(K)}{2} e^{-\frac{1}{2} S(\gamma)-\sqrt{2 S(\gamma) / \boldsymbol{P}(K)}}
$$

where $K$ is the event $\left\{\sup _{t \in[0, T]}|W(t)| \leqslant \delta\right\}$.

The proof of Lemma 3.12 is standard - it follows from a change of measure, as in the proof of Theorem 3.2.1 of [FW12], for example. For convenience we provide a proof at the end of this section, and prove Lemmas 3.9, 3.10 and 3.11 next.

Proof of Lemma 3.9. We need to show that for an interval $\left[r_{1}, r_{2}\right] \subset\left[-\alpha \varepsilon^{2} / 2, \alpha \varepsilon^{2} / 2\right]$,

$$
\inf _{z \in A^{\prime} \cup D^{\prime}} \boldsymbol{P}^{z}\left(Z_{\tau_{0}}^{\varepsilon} \in\left[r_{1}, r_{2}\right] \times\{0\}\right) \geqslant C \frac{\left|r_{2}-r_{1}\right|}{\alpha \varepsilon^{2}} .
$$

Suppose $z \in D^{\prime}$ (the case $z \in A^{\prime}$ is similar but less complicated by the domain geometry). In order to hit $G_{0}$, the process must first hit the boundary of $B\left(0, \alpha \varepsilon^{2}\right)$ which is a ball of radius $\alpha \varepsilon^{2}$, centered at the origin $(0,0)$, since $G_{0} \subset B\left(0, \alpha \varepsilon^{2}\right)$. So, by the strong Markov property, it suffices to show that

$$
\inf _{z \in B\left(0, \varepsilon^{2}\right)} \boldsymbol{P}^{z}\left(Z_{\tau_{0}}^{\varepsilon} \in\left[r_{1}, r_{2}\right] \times\{0\}\right) \geqslant C \frac{\left|r_{2}-r_{1}\right|}{\alpha \varepsilon^{2}} .
$$

Suppose that $\left[r_{1}, r_{2}\right]=\left[r_{0}-\kappa, r_{0}+\kappa\right]$. Let $\ell=\left\{r_{0}\right\} \times\left[-\varepsilon^{2}, 0\right)$ be the vertical line segment of length $\varepsilon^{2}$ below the desired exit interval. Let $T=\varepsilon^{4}, \delta=\varepsilon^{2} / 4$, and let $\gamma$ be a curve parametrized by arc-length such that $\gamma(0)=z$ and the event $\sup _{t \in[0, T]}\left|Z^{\varepsilon}(t)-\gamma(t)\right| \leqslant \delta$ implies that $Z^{\varepsilon}$ hits $\ell$ before $G_{0}$ (one example of such a curve is shown in Figure 4). We can choose such a curve $\gamma$ for which $\left|\gamma^{\prime}\right| \leqslant O\left(\varepsilon^{-2}\right)$, so that the quantity $S(\gamma)$ in Lemma 3.12 is bounded independent of $\varepsilon$ and of $z=\gamma(0) \in B\left(0, \varepsilon^{2}\right)$. Notice also that the set $K$ from Lemma 3.12 satisfies

$$
\boldsymbol{P}(K)=\boldsymbol{P}\left(\sup _{t \in[0, T]}|W(t)| \leqslant \delta\right)=\boldsymbol{P}\left(\sup _{t \in[0,1]}|W(t)| \leqslant \frac{\delta}{\sqrt{T}}\right)
$$

by Brownian scaling. Then since $\delta / \sqrt{T}$ is constant, this probability is bounded below and Lemma 3.12 states the probability that $Z_{t}^{\varepsilon}$ hits $\ell$ before $G_{0}$ is bounded 


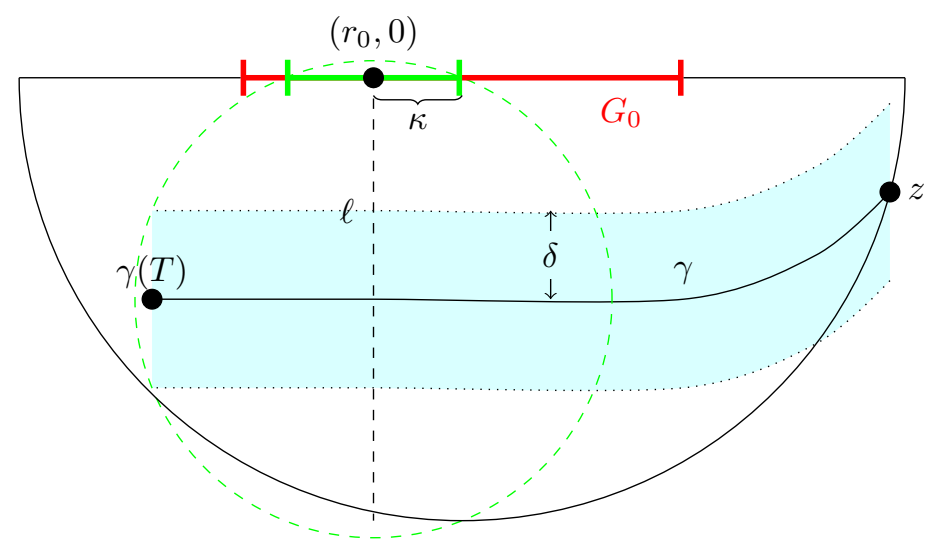

Figure 4 . The curve $\gamma$ starts on $\partial B\left(0, \varepsilon^{2}\right)$, goes through the line $\ell$ while keeping a distance $\delta$ from the gate $G_{0}$.

below (away from zero), independent of $\varepsilon$. By the Markov property it now suffices to finish the proof assuming $z_{0} \in \ell$. Then consider the unique circle with center at $z_{0} \in \ell$ such that the circle intersects $G_{0}$ at the points $\left(r_{0}-\kappa, 0\right)$ and $\left(r_{0}+\kappa, 0\right)$. By symmetry of Brownian motion, the exit distribution on the circle is uniform. The probability that $Z_{\tau_{0}}^{\varepsilon} \in\left[r_{0}-\kappa, r_{0}+\kappa\right]$ is at least the probability of exiting this circle along the arc above $G_{0}$, which is the ratio of the arc length to the circumference. This probability is bounded below by $2 \kappa /\left(\alpha \varepsilon^{2}\right) \gtrsim\left|r_{1}-r_{2}\right| /\left(\alpha \varepsilon^{2}\right)$.

Proof of Lemma 3.10. By the Markov property, Lemma 3.10 will follow from the estimate

$$
\sup _{z \in Q} \boldsymbol{E}^{z} \int_{0}^{\tau_{0}} \mathbf{1}_{Q}\left(Z_{s}^{\varepsilon}\right) d s \leqslant C \varepsilon^{2}|\ln \varepsilon| .
$$

Let $D=\{ \pm \varepsilon / 2\} \times[-\varepsilon, 0]$ be the sides of $Q$, and recall $D^{\prime}$ (defined in (3.17)) denotes the sides of $Q^{\prime}$. We consider two sequences of stopping times, $\zeta_{i}, \eta_{i}$, denoting successive visits of of $Z^{\varepsilon}$ to $G_{0} \cup D^{\prime}$ and $D$ respectively. Precisely, let $\eta_{0}=0$, inductively define

$$
\begin{aligned}
\zeta_{i} & =\inf \left\{s>\eta_{i-1} \mid Z_{s}^{\varepsilon} \in G_{0} \cup D^{\prime}\right\} \\
\eta_{i} & =\inf \left\{s>\zeta_{i} \mid Z_{s}^{\varepsilon} \in D\right\},
\end{aligned}
$$

for $i \in\{1,2, \ldots\}$, and let

$$
M=\min \left\{n \in \mathbb{N} \mid Z_{\zeta_{n}}^{\varepsilon} \in G_{0}\right\} .
$$

Notice that $\zeta_{M}=\tau_{0}$. Using the strong Markov property, and the fact that $Z_{s}^{\varepsilon} \notin Q$ for $s \in\left(\zeta_{i}, \eta_{i}\right)$ for all $i<M$, we obtain

$$
\begin{aligned}
\boldsymbol{E}^{z} \int_{0}^{\tau_{0}} \mathbf{1}_{Q}\left(Z_{s}^{\varepsilon}\right) d s & =\boldsymbol{E}^{z} \sum_{i=1}^{M} \int_{\eta_{i-1}}^{\zeta_{i}} \mathbf{1}_{Q}\left(Z_{s}^{\varepsilon}\right) d s=\boldsymbol{E}^{z} \sum_{i=1}^{M} \boldsymbol{E}^{Z_{\eta_{i-1}}^{\varepsilon}} \int_{0}^{\zeta_{1}} \mathbf{1}_{Q}\left(Z_{s}^{\varepsilon}\right) d s \\
& \leqslant\left(\boldsymbol{E}^{z} M\right)\left(\sup _{d \in D} \boldsymbol{E}^{d} \int_{0}^{\zeta_{1}} \mathbf{1}_{Q}\left(Z_{s}^{\varepsilon}\right) d s\right)
\end{aligned}
$$


Since $\zeta_{1}$ is bounded by the exit time of a one dimensional Brownian motion (the first coordinate of $Z^{\varepsilon}$ ) from an interval of length $3 \varepsilon / 2$, we know

$$
\sup _{d \in D} \boldsymbol{E}^{d} \zeta_{1} \leqslant C \varepsilon^{2} \text {. }
$$

Using this in (3.29) shows

$$
\boldsymbol{E}^{z} \int_{0}^{\tau_{0}} \mathbf{1}_{Q}\left(Z_{s}^{\varepsilon}\right) d s \leqslant C \varepsilon^{2} \boldsymbol{E}^{z} M .
$$

We now estimate $\boldsymbol{E}^{z} M$. Notice that

$$
\begin{aligned}
& \boldsymbol{P}^{z}(M \geqslant n)=\boldsymbol{P}^{z}\left(Z_{\zeta_{1}}^{\varepsilon} \notin G_{0}, Z_{\zeta_{2}}^{\varepsilon} \notin G_{0}, \ldots, Z_{\zeta_{n}}^{\varepsilon} \notin G_{0}\right) \\
& =\boldsymbol{E}^{z}\left(\mathbf{1}_{\left\{Z_{\zeta_{1}}^{\varepsilon} \notin G_{0}, Z_{\zeta_{2}}^{\varepsilon} \notin G_{0}, \ldots, Z_{\zeta_{n-1}}^{\varepsilon} \notin G_{0}\right\}} \boldsymbol{P}^{\left.Z_{\eta_{n-1}}^{\varepsilon}\left(Z_{\zeta_{1}}^{\varepsilon} \notin G_{0}\right)\right)}\right. \\
& \leqslant \boldsymbol{P}^{z}\left(Z_{\zeta_{1}}^{\varepsilon} \notin G_{0}, Z_{\zeta_{2}}^{\varepsilon} \notin G_{0}, \ldots, Z_{\zeta_{n-1}}^{\varepsilon} \notin G_{0}\right)\left(\sup _{d \in D} \boldsymbol{P}^{d}\left(Z_{\zeta_{1}}^{\varepsilon} \notin G_{0}\right)\right) \\
& =\boldsymbol{P}^{z}(M \geqslant n-1)\left(\sup _{d \in D} \boldsymbol{P}^{d}\left(Z_{\zeta_{1}}^{\varepsilon} \notin G_{0}\right)\right) .
\end{aligned}
$$

Thus, by induction

$$
\boldsymbol{P}^{z}(M \geqslant n) \leqslant\left(\sup _{d \in D} \boldsymbol{P}^{d}\left(Z_{\zeta_{1}}^{\varepsilon} \notin G_{0}\right)\right)^{n} .
$$

Now we claim that there exist a constant $c_{0}>0$, independent of $\varepsilon$, such that

$$
\sup _{d \in D} \boldsymbol{P}^{d}\left(Z_{\zeta_{1}}^{\varepsilon} \notin G_{0}\right)<1-\frac{c_{0}}{|\ln \varepsilon|} .
$$

This is the key step in the proof. Once established, it implies

$$
\boldsymbol{E}^{z} M=\sum_{n=1}^{\infty} \boldsymbol{P}^{z}(M \geqslant n) \leqslant \sum_{n=1}^{\infty}\left(1-\frac{c_{0}}{|\ln \varepsilon|}\right)^{n-1}=\frac{|\ln \varepsilon|}{c_{0}},
$$

which when combined with with (3.30) yields

$$
\sup _{z \in D} \boldsymbol{E}^{z} \int_{0}^{\tau_{1}} \mathbf{1}_{Q}\left(Z_{s}^{\varepsilon}\right) d s \leqslant \frac{C \varepsilon^{2}|\ln \varepsilon|}{c_{0}} .
$$

This proves (3.28) and finishes the proof of Lemma 3.10.

Thus it only remains to prove (3.31). We will prove it by showing

$$
\inf _{z \in D} \boldsymbol{P}^{z}\left(Z_{\zeta_{1}}^{\varepsilon} \in G_{0}\right)>\frac{c_{0}}{|\ln \varepsilon|} .
$$

We will prove this in three stages. First, by scaling, it is easy to see that the probability that starting from $D$ the process $Z^{\varepsilon}$ hits $B(0, \varepsilon / 4)$ before $D^{\prime}$ with probability $c_{0}>0$. Next, using the explicit Greens function in an annulus we show that the probability that starting from $B(0, \varepsilon / 4)$, the process $Z^{\varepsilon}$ hits $B\left(0, \alpha \varepsilon^{2}\right)$ before exiting $B(0, \varepsilon / 2)$ with probability $c_{0} /|\ln \varepsilon|$. Finally, by scaling, it again follows that that starting from $B\left(0, \alpha \varepsilon^{2}\right)$ the process $Z^{\varepsilon}$ hits $G_{0}$ before exiting $B\left(0,2 \alpha \varepsilon^{2}\right)$ with probability $c_{0}>0$.

For the first stage, consider the stopping times

$$
\begin{aligned}
& \sigma_{\varepsilon / 4}=\inf \left\{t>0 \mid Z_{t}^{\varepsilon} \in B\left(0, \frac{\varepsilon}{4}\right)\right\}, \\
& \sigma_{D^{\prime}}=\inf \left\{t>0 \mid Z_{t}^{\varepsilon} \in D^{\prime}\right\} .
\end{aligned}
$$


By rescaling, it immediately follows that

$$
\inf _{z \in D} \boldsymbol{P}\left(\sigma_{\varepsilon / 4}<\sigma_{D^{\prime}} \mid Z_{0}^{\varepsilon}=z\right) \geqslant p_{1},
$$

for some $p_{1}>0$, independent of $\varepsilon$.

For the second stage suppose for $Z_{0}^{\varepsilon} \in \partial B(0, \varepsilon / 4)$. Consider the stopping times $\sigma_{\alpha \varepsilon^{2}}$ and $\sigma_{\varepsilon / 2}$ defined by

$$
\begin{aligned}
\sigma_{\alpha \varepsilon^{2}} & =\inf \left\{t>0 \mid Z_{t}^{\varepsilon} \in \partial B\left(0, \varepsilon^{2}\right)\right\}, \\
\sigma_{\varepsilon / 2} & =\inf \left\{t>0 \mid Z_{t}^{\varepsilon} \in \partial B(0, \varepsilon / 2)\right\} .
\end{aligned}
$$

The function

$$
f(z)=\frac{\ln (2|z| / \varepsilon)}{\ln (2 \alpha \varepsilon)}
$$

is harmonic in $B(0, \varepsilon / 2)-B\left(0, \alpha \varepsilon^{2}\right)$ and satisfies $f=1$ on $\partial B\left(0, \alpha \varepsilon^{2}\right)$, and $f=0$ on $\partial B(0, \varepsilon / 2)$. This implies that for all $z \in B(0, \varepsilon / 4)$ we have

$$
\boldsymbol{P}^{z}\left(\sigma_{\alpha \varepsilon^{2}}<\sigma_{\varepsilon / 2}\right)=f(z)=\frac{\ln (1 / 2)}{\ln (2 \varepsilon)} .
$$

Finally, for the last stage, let $\sigma_{2 \alpha \varepsilon^{2}}$ be the first time $Z^{\varepsilon}$ exits $B\left(0,2 \alpha \varepsilon^{2}\right)$. By scaling, it immediately follows that for all $z \in \partial B\left(0, \alpha \varepsilon^{2}\right)$

$$
\boldsymbol{P}^{z}\left(\tau_{0}<\sigma_{2 \alpha \varepsilon^{2}}\right) \geqslant p_{2},
$$

for some constant $p_{2}>0$, independent of $\varepsilon$.

The strong Markov property and (3.34), (3.35), and (3.36) imply

$$
\inf _{z \in D} \boldsymbol{P}\left(Z_{\zeta_{1}}^{\varepsilon} \in G_{0} \mid Z_{0}^{\varepsilon}=z\right) \geqslant p_{1} \cdot \frac{\ln (1 / 2)}{\ln (2 \alpha \varepsilon)} \cdot p_{2} .
$$

By the time-homogeneity of the Markov process $Z^{\varepsilon}$, this establishes (3.33), finishing the proof.

Proof of Lemma 3.11. To estimate the local time term, consider the function

$$
w(x, y)= \begin{cases}\alpha \varepsilon^{2}-y, & y \in\left[0, \alpha \varepsilon^{2}\right], \\ \alpha \varepsilon^{2}, & \text { otherwise, }\end{cases}
$$

which satisfies $\partial_{y} w\left(x, 0^{+}\right)-\partial_{y} w\left(x, 0^{-}\right)=-1$ for $x \in\left[-\alpha \varepsilon^{2} / 2, \alpha \varepsilon^{2} / 2\right]$. Let $\tau_{A^{\prime}}$ be the first hitting time to the set $A^{\prime}$, where we know $w=0$. Using Itô's formula we obtain

Clearly $\tau_{A^{\prime}} \geqslant \tau_{0}^{\prime}$, and so

$$
\boldsymbol{E}^{z} L_{\tau_{A^{\prime}}}^{G_{0}}=w(z), \quad z \in G_{0} .
$$

$$
\sup _{z \in G_{0}} \boldsymbol{E}^{z} L_{\tau_{0}^{\prime}}^{G_{0}} \leqslant \sup _{z \in G_{0}} \boldsymbol{E}^{z} L_{\tau_{A^{\prime}}}^{G_{0}}=\alpha \varepsilon^{2} .
$$

Next, we estimate the term

$$
\sup _{z \in G_{0}} \boldsymbol{E}^{z} \int_{0}^{\tau_{0}^{\prime}} \mathbf{1}_{Q}\left(Z_{s}^{\varepsilon}\right) d s .
$$

Let $\tau_{D^{\prime}}=\inf \left\{t>0 \mid Z_{t}^{\varepsilon} \in D^{\prime}\right\}$, so that $\tau_{D^{\prime}} \geqslant \tau_{0}^{\prime}$. Let $H=\left\{(x, y) \in \mathbb{R}^{2} \mid y=-\varepsilon\right\}$ denote the bottom boundary of $\Omega_{0}$, and let $H^{\prime}=[-3 \varepsilon / 4,3 \varepsilon / 4] \times\{-\varepsilon\}=\bar{Q}^{\prime} \cap H$. We now consider repeated visits to $H^{\prime}$ before hitting $D^{\prime}$. For this, define the stopping times $\left\{\zeta_{k}\right\}_{k=0}^{\infty}$ inductively by

$$
\zeta_{0}=\inf \left\{t>0 \mid Z_{t}^{\varepsilon} \in H\right\}
$$




$$
\zeta_{k}=\inf \left\{t \geqslant \zeta_{k-1}+\varepsilon^{2} \mid Z_{t}^{\varepsilon} \in H\right\}, \quad \text { for } k=1,2,3, \ldots,
$$

and define

$$
M=\min \left\{k \in \mathbb{N} \mid Z_{\zeta_{k}}^{\varepsilon} \in H-H^{\prime}\right\} .
$$

Observe that if $Z_{0}^{\varepsilon} \in G_{0}$, then $\tau_{D^{\prime}} \leqslant \zeta_{M}$. Indeed, since $Z_{\zeta_{M}}^{\varepsilon} \in H-H^{\prime}$ and trajectories of process $Z^{\varepsilon}$ is continuous, they must must have passed through the set $D^{\prime}$ at some time before $\zeta_{M}$.

Now, to bound (3.37) we observe

$$
\int_{0}^{\tau_{0}^{\prime}} \mathbf{1}_{Q}\left(Z_{s}^{\varepsilon}\right) d s \leqslant \int_{0}^{\zeta_{0}} \mathbf{1}_{Q}\left(Z_{s}^{\varepsilon}\right) d s+\sum_{k=1}^{M} \int_{\zeta_{k-1}}^{\zeta_{k}} \mathbf{1}_{Q}\left(Z_{s}^{\varepsilon}\right) d s .
$$

On the event $\{M>k-1\}$ we must have $Z_{\zeta_{k-1}}^{\varepsilon} \in H^{\prime}$. Using this observation, the strong Markov property, and the time-homogeneity of the process, we see that for any $z \in G_{0}$ we have

$$
\begin{aligned}
\boldsymbol{E}^{z} \int_{0}^{\tau_{0}^{\prime}} \mathbf{1}_{Q}\left(Z_{s}^{\varepsilon}\right) d s & \leqslant \boldsymbol{E}^{z} \int_{0}^{\zeta_{0}} \mathbf{1}_{Q}\left(Z_{s}^{\varepsilon}\right) d s+\boldsymbol{E}^{z} \sum_{k=1}^{M} \int_{\zeta_{k-1}}^{\zeta_{k}} \mathbf{1}_{Q}\left(Z_{s}^{\varepsilon}\right) d s \\
& =\boldsymbol{E}^{z} \int_{0}^{\zeta_{0}} \mathbf{1}_{Q}\left(Z_{s}^{\varepsilon}\right) d s+\boldsymbol{E}^{z} \sum_{k=1}^{M} \boldsymbol{E}^{Z_{\zeta_{k-1}}^{\varepsilon}} \int_{\zeta_{0}}^{\zeta_{1}} \mathbf{1}_{Q}\left(Z_{s}^{\varepsilon}\right) d s \\
& \leqslant \boldsymbol{E}^{z} \int_{0}^{\zeta_{0}} \mathbf{1}_{Q}\left(Z_{s}^{\varepsilon}\right) d s+\boldsymbol{E}^{z} \sum_{k=1}^{M} \sup _{z^{\prime} \in H^{\prime}} \boldsymbol{E}^{z^{\prime}} \int_{\zeta_{0}}^{\zeta_{1}} \mathbf{1}_{Q}\left(Z_{s}^{\varepsilon}\right) d s \\
& =\boldsymbol{E}^{z} \int_{0}^{\zeta_{0}} \mathbf{1}_{Q}\left(Z_{s}^{\varepsilon}\right) d s+\left(\boldsymbol{E}^{z} M\right) \sup _{z^{\prime} \in H^{\prime}} \boldsymbol{E}^{z^{\prime}} \int_{\zeta_{0}}^{\zeta_{1}} \mathbf{1}_{Q}\left(Z_{s}^{\varepsilon}\right) d s .
\end{aligned}
$$

We now bound the right hand side of (3.39). Note

$$
\boldsymbol{E}^{z} M=\sum_{j=1}^{\infty} \boldsymbol{P}^{z}(M \geqslant j)=\sum_{j=1}^{\infty} \boldsymbol{P}^{z}\left(Z_{\zeta_{0}}^{\varepsilon} \in H^{\prime}, Z_{\zeta_{1}}^{\varepsilon} \in H^{\prime}, \ldots, Z_{\zeta_{j-1}}^{\varepsilon} \in H^{\prime}\right)
$$

By the Markov property

$$
\begin{aligned}
& \boldsymbol{P}^{z}\left(Z_{\zeta_{i+1}}^{\varepsilon} \in H^{\prime}, Z_{\zeta_{i}}^{\varepsilon} \in H^{\prime}\right)=\boldsymbol{E}^{z}\left(\mathbf{1}_{Z_{\zeta_{i}}^{\varepsilon} \in H^{\prime}} \boldsymbol{P}^{Z_{\zeta_{i}}^{\varepsilon}}\left(Z_{\zeta_{1}}^{\varepsilon} \in H^{\prime}\right)\right) \\
& \leqslant\left(\sup _{z^{\prime} \in H^{\prime}} \boldsymbol{P}^{z^{\prime}}\left(Z_{\zeta_{1}}^{\varepsilon} \in H^{\prime}\right)\right) \boldsymbol{P}^{z}\left(Z_{\zeta_{i}}^{\varepsilon} \in H^{\prime}\right)
\end{aligned}
$$

Now using Lemma 3.12 and the fact that $\zeta_{1} \geqslant \varepsilon^{2}$, one can show that

$$
\sup _{z^{\prime} \in H^{\prime}} \boldsymbol{P}^{z^{\prime}}\left(Z_{\zeta_{1}}^{\varepsilon} \in H^{\prime}\right) \leqslant 1-c_{0}
$$

for some constant $c_{0}>0$, independent of $\varepsilon$. Combining this with (3.41) and using induction we obtain

$$
\sum_{j=1}^{\infty} \boldsymbol{P}^{z}\left(Z_{\zeta_{0}}^{\varepsilon} \in H^{\prime}, Z_{\zeta_{1}}^{\varepsilon} \in H^{\prime}, \ldots, Z_{\zeta_{j-1}}^{\varepsilon} \in H^{\prime}\right) \leqslant \sum_{j=1}^{\infty}\left(1-c_{0}\right)^{j-1}
$$

Thus, using (3.40) we see

$$
\boldsymbol{E}^{z} M \leqslant \frac{1}{c_{0}}
$$


Using this in (3.39) we have

$$
\begin{aligned}
\boldsymbol{E}^{z} \int_{0}^{\tau_{0}^{\prime}} \mathbf{1}_{Q}\left(Z_{s}^{\varepsilon}\right) d s & \leqslant \boldsymbol{E}^{z} \int_{0}^{\zeta_{0}} \mathbf{1}_{Q}\left(Z_{s}^{\varepsilon}\right) d s+\frac{1}{c_{0}} \sup _{z^{\prime} \in H^{\prime}} \boldsymbol{E}^{z^{\prime}} \int_{\zeta_{0}}^{\zeta_{1}} \mathbf{1}_{Q}\left(Z_{s}^{\varepsilon}\right) d s \\
& \leqslant \boldsymbol{E}^{z} \int_{0}^{\zeta_{0}} \mathbf{1}_{Q}\left(Z_{s}^{\varepsilon}\right) d s+\frac{1}{c_{0}}\left(\varepsilon^{2}+\sup _{z^{\prime} \in \Omega} \boldsymbol{E}^{z^{\prime}} \int_{0}^{\zeta_{0}} \mathbf{1}_{Q}\left(Z_{s}^{\varepsilon}\right) d s\right) .
\end{aligned}
$$

To bound this, consider the function

$$
v(x, y)= \begin{cases}\frac{1}{2}\left(\varepsilon^{2}-y^{2}\right), & y \in[-\varepsilon, 0], \\ \frac{1}{2} \varepsilon^{2}, & y>0 .\end{cases}
$$

and observe that for any $z \in \Omega_{\varepsilon}$,

$$
\boldsymbol{E}^{z} \int_{0}^{\zeta_{0}} \mathbf{1}_{Q}\left(Z_{s}^{\varepsilon}\right) d s \leqslant \boldsymbol{E}^{z} \zeta_{0}=v(z) \leqslant \frac{\varepsilon^{2}}{2} .
$$

Substituting this in (3.42) shows

$$
\boldsymbol{E}^{z} \int_{0}^{\tau_{0}^{\prime}} \mathbf{1}_{Q}\left(Z_{s}^{\varepsilon}\right) d s \leqslant\left(\frac{1}{2}+\frac{3}{2 c_{0}}\right) \varepsilon^{2},
$$

completing the proof.

Finally, for completeness we prove Lemma 3.12. The proof is a standard argument using the Girsanov theorem, and can for instance be found in [FW12] (see Theorem 3.2.1, therein).

Proof of Lemma 3.12. Define $Y(t)=W(t)-\gamma(t)$. Let $B(t)$ be an independent Brownian motion in $\mathbb{R}$ with respect to measure $\boldsymbol{P}$. Let define a new measure $\boldsymbol{Q}$ by

$$
\frac{d \boldsymbol{Q}}{d \boldsymbol{P}}=e^{-\int_{0}^{T} \gamma^{\prime}(s) d B(s)-\frac{1}{2} \int_{0}^{T}\left|\gamma^{\prime}(s)\right|^{2} d s}
$$

Let $\tilde{K}$ be the event $\tilde{K}=\tilde{K}_{T, \delta}=\left\{\sup _{t \in[0, T]}|B(t)| \leqslant \delta\right\}$. Let $S(\gamma)=\int_{0}^{T}\left|\gamma^{\prime}(s)\right|^{2} d s$. According to the Girsanov theorem,

$$
\begin{aligned}
\boldsymbol{P}\left(\sup _{t \in[0, T]}|Y(t)| \leqslant \delta\right) & =\boldsymbol{Q}(\tilde{K}) \\
& =\boldsymbol{E}_{\boldsymbol{P}}\left[\mathbf{1}_{\tilde{K}} e^{-\int_{0}^{T} \gamma^{\prime}(s) d B(s)-\frac{1}{2} \int_{0}^{T}\left|\gamma^{\prime}(s)\right|^{2} d s}\right] \\
& =e^{-\frac{1}{2} S(\gamma)} \boldsymbol{E}_{\boldsymbol{P}}\left[\mathbf{1}_{\tilde{K}} e^{-\int_{0}^{T} \gamma^{\prime}(s) d B(s)}\right]
\end{aligned}
$$

Now, by Chebychev and the Itô isometry,

$$
\boldsymbol{P}\left(\int_{0}^{T} \gamma^{\prime}(s) d B(s) \geqslant \alpha \sqrt{S(\gamma)}\right) \leqslant \frac{1}{\alpha^{2}}
$$

So, if $\frac{1}{\alpha^{2}} \leqslant \frac{1}{2} \boldsymbol{P}(\tilde{K})$, we have

$$
\left.\boldsymbol{P}\left(\sup _{t \in[0, T]}|Y(t)| \leqslant \delta\right) \geqslant e^{-\frac{1}{2} S(\gamma)-\alpha \sqrt{S(\gamma)}}\right) \frac{1}{2} \boldsymbol{P}(\tilde{K})
$$


In particular, by choosing $\alpha=\sqrt{2 / \boldsymbol{P}(\tilde{K})}>0$, we have

$$
\boldsymbol{P}\left(\sup _{t \in[0, T]}|Y(t)| \leqslant \delta\right) \geqslant e^{-\frac{1}{2} S(\gamma)-\sqrt{2 S(\gamma) / \boldsymbol{P}(\tilde{K})}} \frac{1}{2} \boldsymbol{P}(\tilde{K})
$$

Note that $\boldsymbol{P}(\tilde{K})=\boldsymbol{P}(K)$ since $B$ and $W$ have the same law under $\boldsymbol{P}$.

3.6. Local Time on Teeth Boundaries (Lemma 3.7). The last remaining lemma to prove is Lemma 3.7 which is the local time balance within the teeth. We again use the symmetry and geometric series arguments as in the proof of Proposition 3.8 .

Proof of Lemma 3.7. As with (3.6), we will estimate

$$
\begin{aligned}
I_{k} \stackrel{\text { def }}{=} \boldsymbol{E}^{z}\left(\int_{0}^{t} \frac{1}{2} \partial_{x}^{2} f\left(Z_{s}^{\varepsilon}\right) \mathbf{1}_{\left\{Y_{s}^{\varepsilon}>0\right\}} \mathbf{1}_{\left\{\left|X_{s}^{\varepsilon}-\varepsilon k\right|<\varepsilon / 2\right\}} d s\right. \\
\left.+\int_{0}^{t} \partial_{x} f\left(Z_{s}^{\varepsilon}\right) \mathbf{1}_{\left\{\left|X_{s}^{\varepsilon}-\varepsilon k\right|<\varepsilon / 2\right\}} d L_{s}^{ \pm}\right)
\end{aligned}
$$

for any $z \in K \cap \Omega_{\varepsilon}$. As before, Lemma 3.7 will follow if we can show that for any finite $M, \sum_{\varepsilon|k|<M} I_{k}$ vanishes as $\varepsilon \rightarrow 0$. Since there are $O(1 / \varepsilon)$ terms in the sum, it suffices to bound each $I_{k}$ by $o(\varepsilon)$. Without loss of generality, assume $k=0$ and let $T_{0}=\left[-\alpha \varepsilon^{2} / 2, \alpha \varepsilon^{2} / 2\right] \times[0,1]$ denote the tooth centered at $k=0$. Define the function $\tilde{f}: T_{0} \rightarrow \mathbb{R}$ by

$$
\tilde{f}(x, y) \stackrel{\text { def }}{=} f(x, y)-f(0, y)-x \partial_{x} f(0, y),
$$

Note that for all $(x, y) \in T_{0}$ we have

$$
\tilde{f}(0, y)=0, \quad \partial_{x} \tilde{f}(0, y)=0, \quad \text { and } \quad \partial_{x}^{2} \tilde{f}(x, y)=\partial_{x}^{2} f(x, y) .
$$

and hence $\|\tilde{f}\|_{\infty}=O\left(\varepsilon^{4}\right)$. Moreover,

$$
\partial_{y}^{2} \tilde{f}(x, y)=\partial_{y}^{2} f(x, y)-\partial_{y}^{2} f(0, y)-x \partial_{x} \partial_{y}^{2} f(0, y)=O\left(\varepsilon^{4}\right),
$$

assuming $\partial_{y}^{2} f \in C^{1}$, and $\partial_{y} \tilde{f}(x, 0)=O\left(\varepsilon^{4}\right)$ for $x \in\left[-\alpha \varepsilon^{2} / 2, \alpha \varepsilon^{2} / 2\right]$.

We now extend the definition of $\tilde{f}$ continuously outside of $T_{0}$ (into the spine) to a $O\left(\varepsilon^{2}\right)$ neighborhood of $G$ as follows. Let $\eta(x, y)$ be a smooth, radially-symmetric cutoff function, vanishing outside of $B_{2}(0,0)$ and such that $\eta(z)=1$ for $|z| \leqslant 1$. Then, for $y \leqslant 0$ (i.e. outside the tooth $T_{0}$ ), define

$$
\tilde{f}(x, y) \stackrel{\text { def }}{=} \eta\left(\frac{x}{\alpha \varepsilon^{2}}, \frac{y}{\alpha \varepsilon^{2}}\right)\left(f(x, 0)-f(0,0)-x \partial_{x} f(0,0)\right) .
$$

In this way, $\tilde{f}$ has the additional properties that

(1) $\tilde{f}$ vanishes outside of $T_{0} \cup B_{2 \alpha \varepsilon^{2}}(0,0)$,

(2) $\partial_{y} \tilde{f}=0$ on $(\partial Q)-G$,

(3) The jump in $\partial_{y} \tilde{f}$ across $G$ is $O\left(\varepsilon^{4}\right)$,

(4) $\Delta \tilde{f}=O(1)$ in the region $B_{2 \alpha \varepsilon^{2}}^{-}=\{y \leqslant 0\} \cap B_{2 \alpha \varepsilon^{2}}(0,0)$.

This last point stems from the fact that $\left|f(x, 0)-f(0,0)-x \partial_{x} f(0,0)\right|=O\left(\varepsilon^{4}\right)$. In view of this construction, we see that

$$
I_{0}=\boldsymbol{E}^{z}\left(\int_{0}^{t} \frac{1}{2}\left(\partial_{x}^{2} \tilde{f}+\partial_{y}^{2} \tilde{f}\right)\left(Z_{s}^{\varepsilon}\right) \mathbf{1}_{\left\{Z_{s}^{\varepsilon} \in T_{0}\right\}} d s-\int_{0}^{t} \partial_{x} \tilde{f}\left(Z_{s}^{\varepsilon}\right) \mathbf{1}_{\left\{Z_{s}^{\varepsilon} \in T_{0}\right\}} d L_{s}^{+}\right)
$$




$$
\begin{aligned}
& +\boldsymbol{E}^{z}\left(\int_{0}^{t} \partial_{x} f\left(0, Y_{s}^{\varepsilon}\right) d\left(L_{s}^{-}-L_{s}^{+}\right)\right)+O\left(\varepsilon^{2}\right) t \\
& =R_{1}+R_{2}+O\left(\varepsilon^{2}\right) t
\end{aligned}
$$

Notice how we have introduced the $\partial_{y}^{2} \tilde{f}$ term for the price of $O\left(\varepsilon^{2}\right) t$. We also still have $\partial_{y} \tilde{f}(x, 1)=0$ on the top boundary of the tooth. By Itô's formula applied to $\tilde{f}$, we have

$$
\begin{aligned}
R_{1} & =\boldsymbol{E}^{z}\left[\tilde{f}\left(Z_{t}^{\varepsilon}\right)-\tilde{f}\left(Z_{0}^{\varepsilon}\right)\right]+\boldsymbol{E}^{z}\left(\int_{0}^{t} \partial_{y} \tilde{f}\left(X_{s}^{\varepsilon}, 0\right) d L^{G}\right)+\boldsymbol{E}^{z}\left(\int_{0}^{t} O(1) \mathbf{1}_{B_{2 \alpha \varepsilon^{2}}}\left(Z_{s}\right) d s\right) \\
& =O\left(\varepsilon^{4}\right)+O\left(\varepsilon^{2}\right) \boldsymbol{E}^{z}\left(L_{t}^{G}\right)+O(1) \boldsymbol{E}^{z}\left(\int_{0}^{t} \mathbf{1}_{B_{2 \alpha \varepsilon^{2}}^{-}}\left(Z_{s}\right) d s\right) \\
& =O\left(\varepsilon^{4}\right)+O\left(\varepsilon^{2}\right)+O(1) R_{3},
\end{aligned}
$$

by since $\boldsymbol{E}^{z} L_{t}^{G}=O(1)$ by $(3.11)$.

We now estimate the term $R_{2}$. By symmetry with respect to reflection in the $y$ coordinate, we note that

$$
\boldsymbol{E}^{z^{\prime}}\left(\int_{0}^{t} \partial_{x} f\left(0, Y_{s}^{\varepsilon}\right) d\left(L_{s}^{-}-L_{s}^{+}\right)\right)=0
$$

for any $z^{\prime}=(0, y)$ on the axis of the tooth $T_{0}$. Thus by symmetry and the Markov property, it suffices to estimate

$$
\boldsymbol{E}^{z}\left(\int_{0}^{\tau} \partial_{x} f\left(0, Y_{s}^{\varepsilon}\right) d L_{s}^{+}\right)
$$

where $\tau=\inf \left\{t \mid X_{t}^{\varepsilon}=0\right\}$ is the first time that $Z_{t}^{\varepsilon}$ reaches this $x$-axis $\{0\} \times \mathbb{R}$, and $z$ is to the right of the $y$-axis. Clearly this is bounded by $\left\|\partial_{x} f\right\|_{\infty} \boldsymbol{E}^{z} L_{\tau}^{+}$. Moreover, using $x \wedge \alpha \varepsilon^{2} / 2$ as a test function, we immediately see $\boldsymbol{E}^{z} L_{\tau}^{+} \leqslant \alpha \varepsilon^{2} / 2$. This shows $R_{2}=O\left(\varepsilon^{2}\right)$ as desired.

Finally, we estimate the term

$$
R_{3}=\boldsymbol{E}^{z}\left(\int_{0}^{t} \mathbf{1}_{B_{2 \alpha \varepsilon^{2}}^{-}}\left(Z_{s}\right) d s\right)
$$

where $B_{2 \alpha \varepsilon^{2}}^{-}=\{y \leqslant 0\} \cap B_{2 \varepsilon^{2}}(0,0)$. The geometry of the domain $\Omega_{\varepsilon}$ makes this estimate a little tedious. Since the proof is very similar to the arguments used in the proof of Proposition 3.8, we do not spell out all the details here.

We will show that $R_{3} \leqslant O\left(\varepsilon^{3}|\log (\varepsilon)|\right)$. For this, we first claim

$$
\sup _{z \in \Omega_{\varepsilon} \cap K} \boldsymbol{E}^{z}\left(\int_{0}^{\tau_{4 \alpha \varepsilon^{2}}} \mathbf{1}_{B_{2 \alpha \varepsilon^{2}}^{-}}\left(Z_{s}\right) d s\right) \leqslant O\left(\varepsilon^{4}\right)
$$

where $\tau_{4 \alpha \varepsilon^{2}}=\inf \left\{t \mid Z_{t}^{\varepsilon} \in D_{4 \varepsilon^{2}}^{-}\right\}$, and $D_{4 \alpha \varepsilon^{2}}^{-}=\{y \leqslant 0\} \cap \partial B_{4 \alpha \varepsilon^{2}}(0,0)$. This follows by directly applying Itô's formula with a function $f$ satisfying $\Delta f \leqslant 0$ in $\left.\{y \leqslant 0\} \cap B_{4 \alpha \varepsilon^{2}}(0,0)\right\}$, with $\Delta f \leqslant-c<0$ in $B_{2 \alpha \varepsilon^{2}}^{-}$.

Next, we claim that there is $C>0$ such that

$$
\inf _{z \in D_{4 \alpha \varepsilon^{2}}^{-}} \boldsymbol{P}^{z}\left(\sigma_{\varepsilon / 2} \leqslant \tau_{2 \alpha \varepsilon^{2}}\right) \geqslant \frac{C}{|\log (\varepsilon)|},
$$

where $\sigma_{\varepsilon / 2}=\inf \left\{t|| X_{t}^{\varepsilon} \mid=\varepsilon / 2\right\}$ and $\tau_{2 \alpha \varepsilon^{2}}=\inf \left\{t \mid Z_{t}^{\varepsilon} \in B_{2 \alpha \varepsilon^{2}}^{-}\right\}$. This is the narrow escape asymptotics [HS14], and follows from a direct calculation with the Greens 
function in a manner similar to the proof of (3.31). Finally, we claim that for any $t>0$, there is $C>0$ such that

$$
\inf _{\{|x|=\varepsilon / 2\}} \boldsymbol{P}^{z}\left(\tau_{2 \alpha \varepsilon^{2}} \geqslant t\right) \geqslant C \varepsilon .
$$

This follows from comparison between $X_{t}^{\varepsilon}$ and a standard Brownian motion on $\mathbb{R}$, via Lemma 3.4. Thus, starting from $z \in D_{4 \alpha \varepsilon^{2}}^{-}$, with probability at least $C \varepsilon /|\log (\varepsilon)|$ the process $Z_{t}$ will make a long excursion such that it doesn't return to $B_{2 \alpha \varepsilon^{2}}^{-}$before time $t$. Using the same geometric series argument as in the proof of Lemma 3.10, we have

$$
R_{3} \leqslant C(\log (\varepsilon) / \varepsilon) \sup _{z} \boldsymbol{E}^{z}\left(\int_{0}^{\tau_{4 \alpha \varepsilon^{2}}} \mathbf{1}_{B_{2 \alpha \varepsilon^{2}}^{-}}\left(Z_{s}\right) d s\right)=O\left(\varepsilon^{3}|\log (\varepsilon)|\right),
$$

as claimed.

Finally, combining all these estimates we conclude that for any $k, I_{k}$ (defined in (3.43)) is at most $O\left(\varepsilon^{2}\right)$. Consequently $\sum_{\varepsilon|k|<M} I_{k} \rightarrow 0$ as $\varepsilon \rightarrow 0$, concluding the proof.

3.7. Remarks About Other Scalings. Consider a comb-shaped domain with the general scaling described in Remark 1.5. For clarity, let us suppose that

$$
w_{S}(\varepsilon)=\varepsilon^{\sigma}, \quad \text { and } \quad w_{T}(\varepsilon)=\frac{\alpha \varepsilon^{1+\sigma}}{2},
$$

for some $\sigma>0$. Theorem 1.1, which we have proved already, pertains to the case $\sigma=1$. In the cases $\sigma<1$ and $\sigma>1$, the same arguments may be applied, showing that the limit process is the same as with $\sigma=1$. Only a minor modification of Proposition 3.8 and its supporting lemmas are required, and we sketch those modifications here.

Analogous to the previous definition (3.12), we define the sets

$$
Q=\left[-\frac{\varepsilon}{2}, \frac{\varepsilon}{2}\right] \times\left[-\varepsilon^{\sigma}, 0\right] \quad \text { and } \quad G_{0}=\left\{(x, 0) \mid-\alpha \frac{\varepsilon^{1+\sigma}}{2}<x<\alpha \frac{\varepsilon^{1+\sigma}}{2}\right\} .
$$

Notice that $Q$ is no longer a square if $\sigma \neq 1$. In the case $\sigma>1$, the bound $0 \leqslant u^{\varepsilon} \leqslant C \varepsilon^{2}|\ln \varepsilon|$ in Proposition 3.8 remains unchanged. The proofs of Lemma 3.9, Lemma 3.10, and Lemma 3.11, extend in a straightforward way. In particular, the lower bound in Lemma 3.9 becomes $\rho(z, r) \geqslant \delta /\left(\alpha \varepsilon^{1+\sigma}\right)$. In the proof of (3.33) within Lemma 3.10, the balls $B\left(0, \varepsilon^{\sigma} / 4\right)$ and $B\left(0, \alpha \varepsilon^{1+\sigma}\right)$ fill the roles of $B(0, \varepsilon / 4)$ and $B\left(0, \alpha \varepsilon^{2}\right)$ in the previous proof.

In the case $\sigma \in(0,1)$, the bound on $u^{\varepsilon}$ in Proposition 3.8 becomes $0 \leqslant u^{\varepsilon} \leqslant$ $C \varepsilon^{1+\sigma}|\ln \varepsilon|$. Nevertheless, this bound is still $o(\varepsilon)$, so that the rest of the argument for the proof of Lemma 3.3 proceeds as before. To prove this modification of Proposition 3.8, we can modify Lemma 3.9, Lemma 3.10, and Lemma 3.11, as follows. First, $A^{\prime}$ and $D^{\prime}$ are defined to be the sets

$$
A^{\prime} \stackrel{\text { def }}{=}\left[-\alpha \frac{\varepsilon^{1+\sigma}}{2}, \alpha \frac{\varepsilon^{1+\sigma}}{2}\right] \times\left\{\alpha \varepsilon^{1+\sigma}\right\} \quad \text { and } \quad D^{\prime} \stackrel{\text { def }}{=}\left\{ \pm \varepsilon^{\sigma}\right\} \times\left[-\varepsilon^{\sigma}, 0\right] .
$$

With these definitions, the lower bound of Lemma 3.9 becomes $\rho(z, r) \geqslant \frac{\delta}{\alpha \varepsilon^{1+\sigma}}$. In Lemma 3.10, the analogous bound becomes $O\left(\varepsilon^{1+\sigma} \mid \ln (\varepsilon \mid)\right.$. Here, the logarithmic factor arises in the same way as before. The $\varepsilon^{1+\sigma}$ factor comes from the fact that for a Brownian motion on $\mathbb{R}$, the expected time spent in $[-\varepsilon, \varepsilon]$ before hitting $\pm \varepsilon^{\sigma}$ is $O\left(\varepsilon^{1+\sigma}\right)$. Similarly, the bound in Lemma 3.11 is $O\left(\varepsilon^{1+\sigma}\right)$. Together these imply the $O\left(\varepsilon^{1+\sigma}|\ln \varepsilon|\right)$ upper bound in Proposition 3.8. 


\section{Comb-Shaped Graphs (Theorem 1.7).}

4.1. An SDE Description of $Z^{\varepsilon}$. We begin by constructing the graph diffusion $Z^{\varepsilon}$ on the comb $\mathcal{C}_{\varepsilon}$. Following the approach of Freidlin and Sheu [FS00], let $\mathcal{L}^{\varepsilon}$ be the linear operator defined by

$$
\mathcal{L}^{\varepsilon} f= \begin{cases}\frac{1}{2} \partial_{y}^{2} f & \text { if }(x, y) \in \varepsilon \mathbb{Z} \times(0,1), \\ \frac{1}{2} \partial_{x}^{2} f & \text { if }(x, y) \in \mathbb{R} \times\{0\} .\end{cases}
$$

Let the domain, denoted by $\mathcal{D}\left(\mathcal{L}^{\varepsilon}\right)$, be the set of all functions

$$
f \in C_{0}\left(\Omega_{\varepsilon}\right) \cap C_{b}^{2}\left(\Omega_{\varepsilon}-J_{\varepsilon}\right)
$$

such that $\mathcal{L}^{\varepsilon} f \in C_{0}\left(\Omega_{\varepsilon}\right)$ and

$$
\begin{array}{cl}
\alpha \varepsilon \partial_{y} f(x, 0)+\partial_{x}^{+} f(x, 0)-\partial_{x}^{-} f(x, 0)=0 & \text { for } x \in \varepsilon \mathbb{Z}, \\
\partial_{y} f(x, 1)=0 & \text { for } x \in \varepsilon \mathbb{Z}
\end{array}
$$

The general theory in [EK86, §4.1-4.2] (see also [FW93, Theorem 3.1]) can be used to show the existence of a continuous Fellerian Markov process $Z^{\varepsilon}=\left(X^{\varepsilon}, Y^{\varepsilon}\right)$ that has generator $\mathcal{L}^{\varepsilon}$.

In the teeth, and in between the nodes, it is clear that $Z^{\varepsilon}$ is simply a Brownian motion. The flux conditions (4.2a)-(4.2b) introduce local time terms at junction points and ends of the teeth. This can be stated precisely in terms of an Itô formula as in the following Lemma.

Lemma 4.1. Let $F$ be the set of all functions $f \in C\left(\mathcal{C}_{\varepsilon}\right)$ such that $f$ is smooth on $\mathcal{C}_{\varepsilon}-J_{\varepsilon}$ and all one sided derivatives exist at the junction points $J_{\varepsilon}$. There is a Brownian motion $W$ such that for any for any $f \in F$ we have

$$
\begin{aligned}
d f\left(Z_{t}^{\varepsilon}\right)= & \mathbf{1}_{\left\{Y_{t}^{\varepsilon}=0\right\}} \partial_{x} f\left(Z_{t}^{\varepsilon}\right) d W_{t}+\frac{1}{2} \mathbf{1}_{\left\{Y_{t}^{\varepsilon}=0\right\}} \partial_{x}^{2} f\left(Z_{t}^{\varepsilon}\right) d t \\
& +\mathbf{1}_{\left\{Y_{t}^{\varepsilon}>0\right\}} \partial_{y} f\left(Z_{t}^{\varepsilon}\right) d W_{t}+\frac{1}{2} \mathbf{1}_{\left\{Y_{t}^{\varepsilon}>0\right\}} \partial_{y}^{2} f\left(Z_{t}^{\varepsilon}\right) d t \\
& \frac{1}{2+\alpha \varepsilon}\left(\partial_{x}^{+} f\left(Z_{t}^{\varepsilon}\right)-\partial_{x}^{-} f\left(Z_{t}^{\varepsilon}\right)+\alpha \varepsilon \partial_{y} f\left(Z_{t}^{\varepsilon}\right)\right) d \ell_{t} .
\end{aligned}
$$

Here $\ell$ defined by

$$
\ell_{t}=L_{t}^{Z^{\varepsilon}}\left(J_{\varepsilon}\right)
$$

is the local time of the joint process $Z_{t}^{\varepsilon}=\left(X_{t}^{\varepsilon}, Y_{t}^{\varepsilon}\right)$ about the junction points $\varepsilon \mathbb{Z} \times$ $\{0\}$.

Remark 4.2. The coefficients of each of $\partial_{x}^{-}, \partial_{x}^{+}$and $\partial_{y}$ in the local time term above can heuristically be interpreted the chance that $Z^{\varepsilon}$ enters the teeth.

Proof. We refer the reader to Section 2 (and specifically Lemma 2.3) in Freidlin and Sheu [FS00] where stochastic calculus for graph diffusions is developed in a general setting.

Notice that choosing $f(x, y)=x$ and $f(x, y)=y$ in Lemma 4.1 yields the following SDEs:

$$
\begin{aligned}
& d X_{t}^{\varepsilon}=\mathbf{1}_{\left\{Y_{t}^{\varepsilon}=0\right\}} d W_{t}, \\
& d Y_{t}^{\varepsilon}=\mathbf{1}_{\left\{Y_{t}^{\varepsilon}>0\right\}} d W_{t}+\frac{\alpha \varepsilon}{2+\alpha \varepsilon} d \ell_{t}-d L_{t}^{Y^{\varepsilon}}(1)
\end{aligned}
$$


Note that (4.4a) and (4.4b) are coupled through the local time term $d \ell$, which is the local time of the joint process $Z^{\varepsilon}=\left(X^{\varepsilon}, Y^{\varepsilon}\right)$ at the junction points $J_{\varepsilon}$. We claim that with the additional assumption that the process spends 0 time in junctions, weak uniqueness holds for (4.4a)-(4.4b), and thus this system can in fact be used to characterize the process $Z^{\varepsilon}$. Since this will not be used in this paper, we refer the reader to Engelbert and Peskir [EP14] for the proof of similar results.

4.2. Proof of Convergence (Theorem 1.7). We now prove Theorem 1.7. As with the proof of Theorem 1.1, we need to prove tightness and a "generator estimate". We state the results we require as the following two lemmas.

Lemma 4.3. Let $Z^{\varepsilon}=\left(X^{\varepsilon}, Y^{\varepsilon}\right)$ be the process on the comb-shaped graph $\mathcal{C}_{\varepsilon}$, as defined above. Then for any $T>0$, the family of processes $Z^{\varepsilon}$ is tight on $C\left([0, T] ; \mathbb{R}^{2}\right)$.

Lemma 4.4. Let $A$ be the generator (2.9). If $f \in \mathcal{D}(A)$, and $K \subseteq \Omega_{0}$ is compact as a subset of $\mathbb{R}^{2}$, then

$$
\lim _{\varepsilon \rightarrow 0} \sup _{z \in K \cap \mathcal{C}_{\varepsilon}} \boldsymbol{E}^{z}\left(f\left(Z_{t}^{\varepsilon}\right)-f\left(Z_{0}\right)-\int_{0}^{t} A f\left(Z_{s}^{\varepsilon}\right) d s\right)=0
$$

Proof of Theorem 1.7. Using Lemmas 4.3 and 4.4 as replacements for Lemmas 3.1 and 3.3 respectively, the proof of Theorem 1.7 is identical to that of Theorem 1.1.

The remainder of this section is devoted to proving Lemmas 4.3 and 4.4.

Proof of Lemma 4.3. We write both $X^{\varepsilon}$ and $Y^{\varepsilon}$ as time-changed Brownian motions as follows. Let $S(t)=\int_{0}^{t} \mathbf{1}_{\left\{Y_{s}^{\varepsilon}=0\right\}} d s$. Then letting $S^{-1}(t)$ be the right-continuous inverse, by the Dambis-Dubins-Schwartz time change theorem (see for instance [KS91, Section 3.4.B]), $\bar{W}_{t}=X_{S^{-1}(t)}^{\varepsilon}$ is a Brownian motion and $X_{t}^{\varepsilon}=\bar{W}_{S(t)}$. Similarly we can time change $Y^{\varepsilon}$ using $R(t)=\int_{0}^{t} \mathbf{1}_{\left\{Y_{t}^{\varepsilon}>0\right\}} d s$. Equation (4.4b) tells us that $\bar{B}_{t}=Y_{R^{-1}(t)}^{\varepsilon}$ satisfies

$$
d \bar{B}_{t}=d \tilde{B}_{t}+d L_{t}^{\bar{B}}(0)-d L_{t}^{\bar{B}}(1) .
$$

where $\tilde{B}_{t}$ is a Brownian motion and hence $\bar{B}_{t}$ is a doubly-reflected Brownian motion on $[0,1]$ such that $Y_{t}^{\varepsilon}=\bar{B}_{R(t)}$. Since $S(t)-S(s) \leqslant t-s$ and $R(t)-R(s) \leqslant t-s$ holds with probability one, the moduli of continuity of $X^{\varepsilon}$ and $Y^{\varepsilon}$ over $[0, T]$ are no more than those of $\bar{W}$ and $\bar{B}$ over $[0, T]$, respectively. This implies tightness.

Proof of Lemma 4.4. We claim for any $k \in \mathbb{N}$ we have

$$
L^{Z^{\varepsilon}}(\varepsilon k, 0)=L^{X^{\varepsilon}}(\varepsilon k, 0)+L^{Y^{\varepsilon}}(\varepsilon k, 0), \quad \text { and } \quad L^{Y^{\varepsilon}}(\varepsilon k, 0)=\frac{\alpha \varepsilon}{2} L^{X^{\varepsilon}}(\varepsilon k, 0) .
$$

The first equality is immediate from the definition, and the second equality is proved in [FS00]. (The second equality can also be deduced the independent excursion construction in Section 5, below). Consequently

$$
L^{Z^{\varepsilon}}(\varepsilon k, 0)=\frac{2+\alpha \varepsilon}{2} L^{X^{\varepsilon}}(\varepsilon k, 0)=\frac{2+\alpha \varepsilon}{\alpha \varepsilon} L^{Y^{\varepsilon}}(\varepsilon k, 0) .
$$

For any $f \in \mathcal{D}(A)$, Lemma 4.1 gives

$$
f\left(Z_{t}^{\varepsilon}\right)-f\left(Z_{0}^{\varepsilon}\right)=\int_{0}^{t} \partial_{y} f\left(Z_{s}^{\varepsilon}\right) \mathbf{1}_{\left\{Y_{s}^{\varepsilon}>0\right\}} d Y_{s}^{\varepsilon}+\int_{0}^{t} \partial_{x} f\left(Z_{s}^{\varepsilon}\right) \mathbf{1}_{\left\{Y_{s}^{\varepsilon}=0\right\}} d X_{s}^{\varepsilon}
$$




$$
\begin{gathered}
+\int_{0}^{t} \frac{1}{2} \partial_{y}^{2} f\left(Z_{s}^{\varepsilon}\right) \mathbf{1}_{\left\{Y_{s}^{\varepsilon}>0\right\}}+\frac{1}{2} \partial_{x}^{2} f\left(Z_{s}^{\varepsilon}\right) \mathbf{1}_{\left\{Y_{s}^{\varepsilon}=0\right\}} d s \\
+\sum_{k \in \mathbb{Z}}\left(\frac{\alpha \varepsilon}{2+\alpha \varepsilon} \partial_{y} f(\varepsilon k, 0)+\frac{1}{2+\alpha \varepsilon}\left(\partial_{x}^{+} f(\varepsilon k, 0)-\partial_{x}^{-} f(\varepsilon k, 0)\right)\right) L_{t}^{Z^{\varepsilon}}(\varepsilon k, 0) .
\end{gathered}
$$

The first integral on the right of equation (4.6) can be rewritten as

$$
\begin{aligned}
\int_{0}^{t} \partial_{y} f\left(Z_{s}^{\varepsilon}\right) \mathbf{1}_{\left\{Y_{s}^{\varepsilon}>0\right\}} d Y_{s}^{\varepsilon} & =\int_{0}^{t} \partial_{y} f\left(Z_{s}^{\varepsilon}\right) \mathbf{1}_{\left\{Y_{s}^{\varepsilon}>0\right\}} d W_{s}-\int_{0}^{t} \partial_{y} f\left(X_{s}^{\varepsilon}, 1\right) d L_{s}^{Y^{\varepsilon}}(1) \\
& =\int_{0}^{t} \partial_{y} f\left(Z_{s}^{\varepsilon}\right) \mathbf{1}_{\left\{Y_{s}^{\varepsilon}>0\right\}} d W_{s} .
\end{aligned}
$$

Here we used the fact that $\partial_{y} f(x, 1)=0$ for any $f \in \mathcal{D}(A)$.

Returning to (4.6), we note that $f \in C^{2}(\mathbb{R} \times\{0\})$ implies $\partial_{x}^{+} f(\varepsilon k, 0)=\partial_{x}^{-} f(\varepsilon k, 0)$. Thus for $(x, y) \in K \cap \mathcal{C}_{\varepsilon}$, taking expectations on both sides and using (4.5) gives

$$
\begin{aligned}
\boldsymbol{E}^{(x, y)} & \left(f\left(Z_{t}^{\varepsilon}\right)-f\left(Z_{0}^{\varepsilon}\right)-\int_{0}^{t} A f\left(Z_{s}^{\varepsilon}\right) d s\right) \\
= & \frac{1}{2} \boldsymbol{E}^{(x, y)}\left(\int_{0}^{t} \partial_{y}^{2} f\left(Z_{s}^{\varepsilon}\right) \mathbf{1}_{\left\{Y_{s}^{\varepsilon}>0\right\}}+\partial_{x}^{2} f\left(Z_{s}^{\varepsilon}\right) \mathbf{1}_{\left\{Y_{s}^{\varepsilon}=0\right\}}-\partial_{y}^{2} f\left(Z_{s}^{\varepsilon}\right) d s\right. \\
& \left.\quad+\varepsilon \sum_{k \in \mathbb{Z}} \partial_{y} f(\varepsilon k, 0) L_{t}^{X^{\varepsilon}}(\varepsilon k, 0)\right) \\
= & \frac{\alpha}{2} \boldsymbol{E}^{(x, y)}\left(-\int_{0}^{t} \partial_{y} f\left(X_{s}^{\varepsilon}, 0\right) \mathbf{1}_{\left\{Y_{s}^{\varepsilon}=0\right\}} d s+\varepsilon \sum_{k \in \mathbb{Z}} \partial_{y} f(\varepsilon k, 0) L_{t}^{X^{\varepsilon}}(\varepsilon k, 0)\right) \\
= & I+I I,
\end{aligned}
$$

where

$$
\begin{gathered}
I \stackrel{\text { def }}{=} \frac{\alpha}{2} \sum_{k \in \mathbb{Z}} \boldsymbol{E}^{(x, y)} \int_{0}^{t}\left(\partial_{y} f(\varepsilon k, 0)-\partial_{y} f\left(X_{s}^{\varepsilon}, 0\right)\right) \mathbf{1}_{\left\{Y_{s}^{\varepsilon}=0,\left|X_{s}^{\varepsilon}-\varepsilon k\right|<\frac{\varepsilon}{2}\right\}} d s, \\
I I \stackrel{\text { def }}{=} \frac{\alpha}{2} \sum_{k \in \mathbb{Z}} \partial_{y} f(\varepsilon k, 0) \boldsymbol{E}^{(x, y)}\left(\varepsilon L_{t}^{X^{\varepsilon}}-\int_{0}^{t} \mathbf{1}_{\left\{Y_{s}^{\varepsilon}=0,\left|X_{s}^{\varepsilon}-\varepsilon k\right|<\frac{\varepsilon}{2}\right\}} d s\right) .
\end{gathered}
$$

Note that there exists Brownian motion $W$ such that $X_{t}^{\varepsilon}=W_{S(t)}$ where $S(t)$, defined by

$$
S(t) \stackrel{\text { def }}{=} \int_{0}^{t} \mathbf{1}_{\left\{Y^{\varepsilon}(s)=0\right\}} d s,
$$

is the amount of time the joint process spends on the spine of the comb up to time $t$. To estimate $I$, for any $\delta>0$ we choose sufficiently large compact set $C \subset \mathbb{R}$ such that

$$
\sup _{(x, y) \in K} \boldsymbol{E}^{x}\left(\int_{0}^{t} \mathbf{1}_{\left\{W_{s} \notin C\right\}} d s\right)<\frac{\delta}{\left\|\partial_{y} f\right\|_{\infty}} .
$$

Then since $S(s) \leqslant s$, it follows that

$$
\boldsymbol{P}^{x}\left(X_{s}^{\varepsilon} \notin C\right) \leqslant \boldsymbol{P}^{x}\left(W_{s} \notin C\right)
$$

and so the above estimate can be applied for $X^{\varepsilon}$ independent of $\varepsilon$. Then use uniform continuity of $\partial_{y} f$ in $C$ along with the above estimate.

In order to estimate $I I$, we again use the above representation to see 


$$
\begin{aligned}
\boldsymbol{E}^{(x, y)}\left|\varepsilon L_{t}^{X^{\varepsilon}}(\varepsilon k, 0)-\int_{0}^{t} \mathbf{1}_{\left\{Y_{s}^{\varepsilon}=0,\left|X_{s}^{\varepsilon}-\varepsilon k\right|<\frac{\varepsilon}{2}\right\}} d s\right| \\
=\boldsymbol{E}^{x}\left|\varepsilon L_{S(t)}^{W}(\varepsilon k)-\int_{0}^{S(t)} \mathbf{1}_{\left\{\left|W_{s}-\varepsilon k\right|<\frac{\varepsilon}{2}\right\}} d s\right|,
\end{aligned}
$$

where $S(t)$, defined by

$$
S(t) \stackrel{\text { def }}{=} \int_{0}^{t} \mathbf{1}_{\left\{Y^{\varepsilon}(s)=0\right\}} d s,
$$

is the amount of time the joint process spends on the spine of the comb up to time $t$. Thus to show $I I \rightarrow 0$, it suffices to estimate the right hand side of (4.7) as $\varepsilon \rightarrow 0$. Also, by shifting the indices of the sum to compensate, we can assume that $x=0$.

To this end, let $f_{\varepsilon}$ be defined by

$$
f_{\varepsilon}(x) \stackrel{\text { def }}{=} \begin{cases}\varepsilon(\varepsilon k-x)-\frac{\varepsilon^{2}}{4} & \text { if } x<\varepsilon k-\frac{\varepsilon}{2}, \\ (x-\varepsilon k)^{2} & \text { if } \varepsilon k-\frac{\varepsilon}{2} \leqslant x \leqslant \varepsilon k+\frac{\varepsilon}{2}, \\ \varepsilon(x-\varepsilon k)-\frac{\varepsilon^{2}}{4} & \text { if } x>\varepsilon k+\frac{\varepsilon}{2} .\end{cases}
$$

By Ito's formula we have,

$$
\begin{aligned}
& f_{\varepsilon}\left(W_{t}\right)-\varepsilon\left|W_{t}-\varepsilon k\right|-\left(f_{\varepsilon}\left(W_{0}\right)-\varepsilon\left|W_{0}-\varepsilon k\right|\right) \\
& =\int_{0}^{t}\left(f_{\varepsilon}^{\prime}\left(W_{s}\right)-\varepsilon \operatorname{sign}\left(W_{s}-\varepsilon k\right)\right) d W_{s}+\int_{0}^{t} \mathbf{1}_{\left\{\left|W_{s}-\varepsilon k\right|<\frac{\varepsilon}{2}\right\}} d s-\varepsilon L_{t}^{W}(\varepsilon k) .
\end{aligned}
$$

Using the Itô isometry and the inequalities

$$
\begin{gathered}
\left|f_{\varepsilon}(x)-\varepsilon\right| x-\varepsilon k|| \leqslant \frac{\varepsilon^{2}}{4}, \\
\left|f_{\varepsilon}^{\prime}(x)-\varepsilon \operatorname{sign}(x-\varepsilon k)\right| \leqslant \varepsilon \mathbf{1}_{\left[\varepsilon k-\frac{\varepsilon}{2}, \varepsilon k+\frac{\varepsilon}{2}\right]},
\end{gathered}
$$

we obtain

$$
\begin{array}{r}
\boldsymbol{E}^{0}\left|\varepsilon L_{t}^{W}(\varepsilon k)-\int_{0}^{t} \mathbf{1}_{\left\{\left|W_{s}-\varepsilon k\right|<\frac{\varepsilon}{2}\right\}} d s\right| \leqslant \frac{\varepsilon^{2}}{4}+\varepsilon\left(\boldsymbol{E}^{0} \int_{0}^{t} \mathbf{1}_{\left\{\left|W_{s}-\varepsilon k\right|<\frac{\varepsilon}{2}\right\}} d s\right)^{\frac{1}{2}} \\
\leqslant c(t) \varepsilon^{\frac{3}{2}},
\end{array}
$$

since

$$
\boldsymbol{E}^{0} \int_{0}^{t} \mathbf{1}_{\left\{\left|W_{s}-\varepsilon k\right|<\frac{\varepsilon}{2}\right\}} d s=\int_{0}^{t} \boldsymbol{P}^{0}\left(\left|W_{s}-\varepsilon k\right|<\frac{\varepsilon}{2}\right) d s \leqslant c \int_{0}^{t} \frac{\varepsilon}{\sqrt{s}} d s=2 c \varepsilon \sqrt{t} .
$$

We break up the sum in $I I$ and estimate as follows,

$$
I I \leqslant\left\|\partial_{y} f\right\|_{\infty}\left(\sum_{|k|>N / \varepsilon} \boldsymbol{E}^{0}\left[\varepsilon L_{t}^{X^{\varepsilon}}(\varepsilon k, 0)\right]+\int_{0}^{t} \boldsymbol{P}^{0}\left(\left|X_{s}^{\varepsilon}\right|>N-\frac{\varepsilon}{2}\right) d s+\frac{2 N}{\varepsilon} c(t) \varepsilon^{\frac{3}{2}}\right) .
$$

We can again use that $X^{\varepsilon}$ has the same distribution as a Brownian motion with a time change $S(t) \leqslant t$ to replace $X^{\varepsilon}$ with $W$, i.e.

$$
I I \leqslant\left\|\partial_{y} f\right\|_{\infty}\left(\sum_{|k|>N / \varepsilon} \boldsymbol{E}^{0}\left[\varepsilon L_{t}^{W}(\varepsilon k)\right]+\int_{0}^{t} \boldsymbol{P}^{0}\left(\left|W_{s}\right|>N-\frac{\varepsilon}{2}\right) d s+N c(t) \varepsilon^{\frac{1}{2}}\right) .
$$


Setting $N$ sufficiently large and then sending $\varepsilon \rightarrow 0$ gives us $I I \rightarrow 0$ as $\varepsilon \rightarrow 0$. This completes the proof.

\section{Excursion Description on the Comb Graph.}

In this section we describe the how diffusion $Z^{\varepsilon}$ on the comb-shaped graph $\mathcal{C}_{\varepsilon}$ (defined in Section 1.2) can be constructed from the point of view of Itô's excursion theory (c.f. [Itô72,PY07]). We identify the components of $Z^{\varepsilon}$ as a trapped Brownian motion in the framework of Ben Arous et al. [BAC+15], and use this to provide an alternate description of the limiting behavior as $\varepsilon \rightarrow 0$.

5.1. The Excursion Decomposition of $Z^{\varepsilon}$. The trajectories of $Z^{\varepsilon}$ can be decomposed as a sequence of excursions where each excursion starts and ends at the junction points $J_{\varepsilon}=\varepsilon \mathbb{Z} \times\{0\}$, and travels entirely in the teeth, or entirely in the spine. The excursions into the teeth of the comb (excursions of $Y^{\varepsilon}$ into $(0,1]$ while $X^{\varepsilon} \in \varepsilon \mathbb{Z}$ ) should be those of a reflected Brownian motion on $[0,1]$. The excursions into the spine (excursions of $X^{\varepsilon}$ into $\mathbb{R}-\varepsilon \mathbb{Z}$ with $Y^{\varepsilon}=0$ ) should be those of a standard Brownian motion on $\mathbb{R}$ between the points $\varepsilon \mathbb{Z}$. Thus one expects that that by starting with a standard Brownian motion $\bar{X}$ on $\mathbb{R}$ and an independent reflected Brownian motion $\bar{Y}$ on $[0,1]$, we can glue excursions of $\bar{X}$ and $\bar{Y}$ appropriately and obtain the diffusion $Z^{\varepsilon}$ on the comb-shaped graph $\mathcal{C}_{\varepsilon}$. We describe this precisely as follows.

Let $\bar{X}$ be a standard Brownian motion on $\mathbb{R}$ and let $L_{t}^{\bar{X}}(x)$ denote its local time at $x \in \mathbb{R}$. Let $L_{t}^{\bar{X}}(\varepsilon \mathbb{Z})$, defined by

$$
L_{t}^{\bar{X}}(\varepsilon \mathbb{Z}) \stackrel{\text { def }}{=} \sum_{k \in \mathbb{Z}} L_{t}^{\bar{X}}(\varepsilon k)=\lim _{\delta \rightarrow 0} \frac{1}{2 \delta} \int_{0}^{t} \sum_{k \in \mathbb{Z}} \mathbf{1}_{(\varepsilon k-\delta, \varepsilon k+\delta)}\left(\bar{X}_{s}\right) d s,
$$

denote the local time of $\bar{X}$ at the junction points $\varepsilon \mathbb{Z}$. Let $\tau^{\bar{X}, \varepsilon}$ be the rightcontinuous inverse of $L_{t}^{\bar{X}}(\varepsilon \mathbb{Z})$ defined by

$$
\tau^{\bar{X}, \varepsilon}(\ell)=\inf \left\{t>0 \mid L_{t}^{\bar{X}}(\varepsilon \mathbb{Z})>\ell\right\}, \quad \ell \geqslant 0 .
$$

Notice that the functions $t \mapsto L_{t}^{\bar{X}}$ and $\ell \mapsto \tau^{\bar{X}, \varepsilon}(\ell)$ are both non-decreasing.

Let $\bar{Y}$ be a reflected Brownian motion on $[0,1]$ which is independent of $\bar{X}$. As above, let $L^{\bar{Y}}(0)$ be the local time of $\bar{Y}$ about 0 , and let $\tau^{\bar{Y}}$, defined by

$$
\tau^{\bar{Y}}(\ell)=\inf \left\{t>0 \mid L_{t}^{\bar{Y}}(0)>\ell\right\},
$$

be its right-continuous inverse. Given $\alpha \in(0,1)$, we define the random time-changes $\psi^{\bar{X}, \varepsilon}$ and $\psi^{\bar{Y}, \varepsilon}$ by

$$
\psi^{\bar{X}, \varepsilon}(t)=\inf \left\{s>0 \mid s+\tau^{\bar{Y}}\left(\frac{\alpha \varepsilon}{2} L_{s}^{\bar{X}}(\varepsilon \mathbb{Z})\right)>t\right\},
$$

and

$$
\psi^{\bar{Y}, \varepsilon}(t)=\inf \left\{s>0 \mid s+\tau^{\bar{X}, \varepsilon}\left(\frac{2}{\alpha \varepsilon} L_{s}^{\bar{Y}}(0)\right)>t\right\} .
$$

Note both $\psi^{\bar{X}, \varepsilon}$ and $\psi^{\bar{Y}, \varepsilon}$ are continuous and non-decreasing functions of time.

Proposition 5.1. The time-changed process $Z^{\varepsilon}$ defined by

$$
Z^{\varepsilon}(t) \stackrel{\text { def }}{=}\left(\bar{X}\left(\psi^{\bar{X}, \varepsilon}(t)\right), \bar{Y}\left(\psi^{\bar{Y}, \varepsilon}(t)\right)\right)
$$


is the same process $Z^{\varepsilon}$ in Theorem 1.7. Namely it is a Markov process with generator $\mathcal{L}^{\varepsilon}$ (defined in equation (4.1)), and is a weak solution of the system (4.4a)-(4.4b).

This gives an alternate and natural representation of $Z^{\varepsilon}=\left(X^{\varepsilon}, Y^{\varepsilon}\right)$. One can view this time-change representation as the pre-limit analogue of the representation (2.2a) for the limit system (2.3a) - (2.3c). For clarity of presentation, we postpone the proof of Proposition 5.1 to Section 5.4.

Remark 5.2. For simplicity, throughout this section we assume the initial distribution of $Z^{\varepsilon}$ is $\delta_{(0,0)}$, and denote expectations using the symbol $\boldsymbol{E}$ without any superscript. The main results here (in particular Theorem 5.7, below) can directly be adapted to the situation for more general initial distributions as in Theorem 1.7.

5.2. Description as a Trapped Brownian Motion. We now show how this representation can be explained in the framework of trapped Brownian motions as defined by Ben Arous, et al. [BAC+15] (see Definition 4.11 therein). Recall that a trapped Brownian motion, denoted by $B[\mu]$, is a process of the form $B(\psi(t))$ where $B(t)$ is a standard Brownian motion and the time-change $\psi$ has the form

$$
\psi(t)=\inf \left\{s>0 \mid \phi[\mu, B]_{s}>t\right\},
$$

where

$$
\phi[\mu, B]_{s}=\mu\left(\left\{(x, \ell) \in \mathbb{R} \times[0, \infty) \mid L^{B}(x, s) \geqslant \ell\right\}\right),
$$

and $\mu$ is a (random) measure on $\mathbb{R} \times[0, \infty)$ called the trap measure. For example, when $\mu$ is the Lebesgue measure on $\mathbb{R} \times[0, \infty)$, then $\phi[\mu, B]=t$, and $\psi(t)=t$. Alternately, if $\mu$ has an atom at $(x, \ell)$ of mass $r>0$, then $B(\psi(t))$ is trapped at $x$ for a time $r$ at the moment its local time at $x$ exceeds $\ell$.

To use this framework in our scenario, we need to identify a trap measure under which $X^{\varepsilon}$ is a trapped Brownian motion. We do this as follows. First note that the process $\tau_{\ell}^{\bar{Y}}$, appearing in the time change (5.1), is a Lévy subordinator. Thus, there exists a function $\eta^{\bar{Y}}(s):(0, \infty) \rightarrow(0, \infty)$, and a Poisson random measure $N^{\bar{Y}}$ on $[0, \infty) \times[0, \infty)$ with intensity measure $d \ell \times \eta^{\bar{Y}}(s) d s$, such that

$$
\tau_{\ell}^{\bar{Y}}=\int_{[0, \ell]} \int_{[0, \infty)} s N^{\bar{Y}}(d \ell \times d s) .
$$

In the definition of $\psi^{\bar{X}, \varepsilon}(t)$ above, we have

$$
\tau^{\bar{Y}}\left(\frac{\alpha \varepsilon}{2} L_{s}^{\bar{X}}(\varepsilon \mathbb{Z})\right)=\tau^{\bar{Y}}\left(\sum_{k \in \mathbb{Z}} \frac{\alpha \varepsilon}{2} L_{s}^{\bar{X}}(\varepsilon k)\right) .
$$

Because $\tau_{\ell}^{\bar{Y}}$ has stationary, independent increments, this is equal in law to

$$
\tau^{\bar{Y}}\left(\frac{\alpha \varepsilon}{2} L_{s}^{\bar{X}}(\varepsilon \mathbb{Z})\right) \stackrel{d}{=} \sum_{k \in \mathbb{Z}} \tau^{\bar{Y}_{k}}\left(\frac{\alpha \varepsilon}{2} L_{s}^{\bar{X}}(\varepsilon k)\right),
$$

where $\left\{\bar{Y}_{k}\right\}_{k \in \mathbb{Z}}$ are a family of independent reflected Brownian motions on $[0,1]$. That is, the time change $\psi^{\bar{X}, \varepsilon}(t)$ has the same law as

$$
\tilde{\psi}^{\bar{X}, \varepsilon}(t)=\inf \left\{s>0 \mid s+\sum_{k \in \mathbb{Z}} \tau^{\bar{Y}_{k}}\left(\frac{\alpha \varepsilon}{2} L_{s}^{\bar{X}}(\varepsilon k)\right)>t\right\} .
$$


COHN, IYER, NOLEN, AND PEGO

Each of the processes $\tau^{\bar{Y}_{k}}$ can be represented as in (5.3) with independent Poisson random measures $N^{\bar{Y}_{k}}$ :

$$
\tau_{\ell}^{\bar{Y}_{k}}=\int_{[0, \ell]} \int_{[0, \infty)} s N^{\bar{Y}_{k}}(d \ell \times d s)
$$

Since each of the random measures $N^{\bar{Y}_{k}}$ is atomic, we may define $\left\{\left(\ell_{j, k}, s_{j, k}\right)\right\}_{j=1}^{\infty}$ to be the random atoms of $N^{\bar{Y}_{k}}$ by

$$
N^{\bar{Y}_{k}}=\sum_{j=1}^{\infty} \delta_{\left(\ell_{j, k}, s_{j, k}\right)}
$$

Then define a random measure on $\mathbb{R} \times[0, \infty)$ :

$$
\mu^{\bar{X}, \varepsilon}=d x \times d \ell+\sum_{k \in \mathbb{Z}} \sum_{j=1}^{\infty} s_{j, k} \delta_{\left(\varepsilon k,(2 /(\alpha \varepsilon)) \ell_{j, k}\right)}
$$

Returning to (5.4), we now have the representation

$$
s+\sum_{k \in \mathbb{Z}} \tau^{\bar{Y}_{k}}\left(\frac{\alpha \varepsilon}{2} L_{s}^{\bar{X}}(\varepsilon k)\right)=\mu^{\bar{X}, \varepsilon}\left(\left\{(x, \ell) \in \mathbb{R} \times[0, \infty) \mid \ell \leqslant L_{s}^{\bar{X}}(x)\right\}\right) .
$$

It is easy to check that $\mu^{\bar{X}}$ defines a Lévy trap measure, in the sense of [BAC +15$]$, Definition 4.10. This proves the following:

Proposition 5.3. Let $\bar{X}$ be a standard Brownian motion on $\mathbb{R}$ and let $\bar{X}\left[\mu^{\bar{X}, \varepsilon}\right]$ be the trapped Brownian motion (see Definition 4.11 of $[B A C+15]$ ) with trap measure $\mu^{\bar{X}, \varepsilon}$ defined by (5.7). Then the law of $X^{\varepsilon}$ coincides with the law of $\bar{X}\left[\mu^{\bar{X}, \varepsilon}\right]$.

The process $Y^{\varepsilon}$ admits a similar representation as a trapped (reflected) Brownian motion. To this end, we first note that $\tau_{\ell}^{\bar{X}, \varepsilon}$ is also a Lévy subordinator which and can be written as

$$
\tau_{\ell}^{\bar{X}, \varepsilon}=\int_{[0, \ell]} \int_{[0, \infty)} s N^{\bar{X}, \varepsilon}(d \ell \times d s),
$$

where $N^{\bar{X}, \varepsilon}$ is a Poisson random measure on $[0, \infty) \times[0, \infty)$ with intensity measure $d \ell \times \eta^{\bar{X}, \varepsilon}(s) d s$.

Lemma 5.4. The excursion length measure $\eta^{\bar{X}, \varepsilon}$ satisfies the scaling relation,

$$
\eta^{\bar{X}, \varepsilon}(s)=\varepsilon^{-3} \eta^{\bar{X}, 1}\left(\varepsilon^{-2} s\right), \quad s>0 .
$$

Proof. This follows in directly from the standard scaling properties of Brownian motion and its local time, and we omit the details.

Letting $\left\{\left(s_{j}, \ell_{j}\right)\right\}_{j=1}^{\infty}$ denote the atoms of $N^{\bar{X}, \varepsilon}$ we then define a random measure on $[0,1] \times[0, \infty)$ by

$$
\mu^{\bar{Y}, \varepsilon}=d y \times d \ell+\sum_{j=1}^{\infty} s_{j} \delta_{\left(0,(\alpha \varepsilon / 2) \ell_{j}\right)} .
$$

This also is a Lévy Trap Measure in the sense of $[\mathrm{BAC}+15]$ (replacing $\mathbb{R}$ by $[0,1]$ ), and one can easily see that the associated trapped Brownian motion is precisely the process $Y^{\varepsilon}$. 
Proposition 5.5. Let $\bar{Y}$ be a reflected Brownian motion on $[0,1]$, and let $\bar{Y}\left[\mu^{\bar{Y}, \varepsilon}\right]$ be the trapped Brownian motion with trap measure $\mu^{\bar{Y}, \varepsilon}$ defined by (5.9). Then the law of $Y^{\varepsilon}$ coincides with the law of $\bar{Y}\left[\mu^{\bar{Y}, \varepsilon}\right]$.

5.3. Convergence as $\varepsilon \rightarrow 0$. We now use Theorem 6.2 of [BAC +15$]$ to study convergence of $X^{\varepsilon}$ and $Y^{\varepsilon}$ as $\varepsilon \rightarrow 0$. The key step is to establish convergence of the trap measures, as in the following lemma.

Lemma 5.6. Let $N_{*}^{\bar{Y}}$ be a Poisson random measure on $\mathbb{R} \times[0, \infty) \times[0, \infty)$ with intensity measure $d x \times d \ell \times \frac{1}{2} \eta^{\bar{Y}}(s) d s . A s \varepsilon \rightarrow 0$, the random measures $\mu^{\bar{X}, \varepsilon}$ on $\mathbb{R} \times[0, \infty)$, defined in (5.7), converge vaguely in distribution to the random measure $\mu_{*}^{X}$ defined by

$$
\mu_{*}^{X}(A)=\int_{\mathbb{R}} \int_{0}^{\infty} \mathbf{1}_{A}(x, \ell) d x d \ell+\frac{\alpha}{2} \int_{\mathbb{R}} \int_{0}^{\infty} \int_{0}^{\infty} \mathbf{1}_{A}(x, \ell) s N_{*}^{\bar{Y}}(d x \times d \ell \times d s),
$$

for all $A \in \mathcal{B}(\mathbb{R} \times[0, \infty))$. The random measures $\mu^{\bar{Y}, \varepsilon}$ on $[0,1] \times[0, \infty)$, defined in (5.9), converge vaguely in distribution to the measure $\mu_{*}^{Y}$ defined by

$$
\mu_{*}^{Y}(A)=\int_{0}^{1} \int_{0}^{\infty} \mathbf{1}_{A}(y, \ell) d y d \ell+\frac{2}{\alpha} \int_{0}^{\infty} \mathbf{1}_{A}(0, \ell) d \ell \quad A \in \mathcal{B}([0,1] \times[0, \infty)) .
$$

Momentarily postponing the proof of Lemma 5.6, we state the main convergence result in this section.

Theorem 5.7. Let $R(t)$ be a Brownian motion on $[0,1]$ reflected at both endpoints $x=0,1$, and $B$ be a standard Brownian motion on $\mathbb{R}$.

(1) As $\varepsilon \rightarrow 0$, we have $Y^{\varepsilon} \rightarrow Y$ vaguely in distribution on $D([0, \infty))$. Here $Y=R\left[\mu_{*}^{\bar{Y}}\right]$ is a reflected Brownian motion that is sticky at 0 .

(2) As $\varepsilon \rightarrow 0$, we have $X^{\varepsilon} \rightarrow B\left[\mu_{*}^{\bar{X}}\right]$ vaguely in distribution on $D([0, \infty))$. The limit process here may also be written as $B\left((2 / \alpha) L_{t}^{Y}(0)\right)$.

Remark 5.8. Using the SDE methods in Section 4 we are able to obtain joint convergence of the pair $\left(X^{\varepsilon}, Y^{\varepsilon}\right)$ (Theorem 1.7). The trapped Brownian motion framework here, however, only provides convergence of the processes $X^{\varepsilon}$ and $Y^{\varepsilon}$ individually.

Proof of Theorem 5.7. The convergence of $Y^{\varepsilon}$ to $R\left[\mu_{*}^{\bar{Y}}\right]$ is an immediate consequence of Theorem 6.2 of [BAC+15], Lemma 5.6 above, and the properties of Poisson random measures. To identify the limiting process $R\left[\mu_{*}^{\bar{Y}}\right]$ as a sticky Brownian motion, observe that the time change has the form

$$
\mu_{*}^{\bar{Y}}\left(\left\{(y, \ell) \in[0,1] \times[0, \infty) \mid L^{R}(y, s) \geqslant \ell\right\}\right)=s+\frac{2}{\alpha} L^{R}(0, s) .
$$

Thus, the limit process is $Y(t)=R(\psi(t))$ where

$$
\psi(t)=\inf \left\{s>0 \mid s+\frac{2}{\alpha} L^{R}(0, s)>t\right\} .
$$

This is precisely a sticky Brownian motion (see Lemma 2.2).

For the second assertion of the Theorem, the convergence of $X^{\varepsilon}$ to $B\left[\mu_{*}^{\bar{X}}\right]$ is again an immediate consequence of Theorem 6.2 of $[\mathrm{BAC}+15]$ and Lemma 5.6 above. Thus we only need to show that the trapped Brownian motion $B\left[\mu_{*}^{\bar{X}}\right]$ has the same law as the process $X_{t}$ from Theorem 1.7. To compare the two processes, 
we first write them in a similar form. Let $L_{t}^{\bar{B}}(0)$ is the local time of $\bar{B}$ at 0 , and let $\tau_{\ell}^{\bar{B}}$ be the inverse

$$
\tau_{\ell}^{\bar{B}}=\inf \left\{t>0 \mid L_{t}^{\bar{B}}(0)>\ell\right\} .
$$

Then, we have

$$
X_{t}=\bar{W}_{\frac{2}{\alpha} L_{T(t)}^{\bar{B}}}=\bar{W}\left(h^{-1}(t)\right)
$$

where

$$
h^{-1}(t)=\inf \left\{r>0 \mid r+\tau_{r \alpha / 2}^{\bar{B}}>t\right\}
$$

The fact that $(2 / \alpha) L_{T(t)}^{\bar{B}}=h^{-1}(t)$ follows from the definition of $T(t)$, which implies $(2 / \alpha) L_{T(t)}^{\bar{B}}+T(t)=t$.

Therefore, the two processes are

$$
B\left[\mu_{*}^{\bar{X}}\right]=B\left(\phi^{-1}(t)\right) \quad X_{t}=\bar{W}\left(h^{-1}(t)\right)
$$

where $\phi$ is:

$$
\phi(r)=\phi\left[\mu_{*}, B\right]_{r}=\mu_{*}\left(\left\{(x, \ell) \in \mathbb{R} \times[0, \infty) \mid L^{B}(x, r) \geqslant \ell\right\}\right)
$$

If $A_{r}^{B}=\left\{(x, \ell) \in \mathbb{R} \times[0, \infty) \mid L^{B}(x, r) \geqslant \ell\right\}$, then by definition of the trap measure $\mu_{*}$,

$$
\phi(r)=r+\frac{\alpha}{2} \int_{A_{r}^{B} \times[0, \infty)} s N_{*}^{\bar{Y}}(d x \times d \ell \times d s)
$$

The last integral has the same law as $\tau_{r \alpha / 2}^{\bar{B}}$. Hence, $h$ and $\phi$ have the same law.

Notice that $h$ is independent of $\bar{W}$. We claim that $\phi$ is also independent of $B$. To see this observe that the distribution of $\phi(r)$ only depends on $B$ through the volume of $A_{r}^{B}$, which equals $r$ almost surely. This shows $\phi$ is independent of $B$, and thus $B\left(\phi^{-1}(t)\right)$ and $\bar{W}\left(h^{-1}(t)\right)$ have the same law.

It remains to prove Lemma 5.6.

Proof of Lemma 5.6. It suffices to show for rectangles $A=\left[x_{0}, x_{1}\right] \times\left[\ell_{0}, \ell_{1}\right]$ that

$$
\mu^{\bar{X}, \varepsilon}(A) \rightarrow \mu_{*}^{X}(A)
$$

in distribution. We calculate the characteristic function using [Kyp06, Thm 2.7],

$$
\begin{aligned}
\boldsymbol{E}\left[e^{i \beta \mu^{\bar{X}, \varepsilon}(A)}\right] & =\exp \left(i \beta|A|+\sum_{\varepsilon k \in\left[x_{0}, x_{1}\right]} \int_{\frac{\varepsilon}{2} \ell_{0}}^{\frac{\varepsilon}{2} \ell_{1}} \int_{0}^{\infty}\left(1-e^{i \beta s}\right) \eta^{\bar{Y}}(s) d s\right) \\
& =\exp \left(i \beta|A|+\left(\left\lfloor\frac{x_{1}}{\varepsilon}\right\rfloor-\left\lceil\frac{x_{0}}{\varepsilon}\right\rceil\right) \frac{\varepsilon\left(\ell_{1}-\ell_{0}\right)}{2} \int_{0}^{\infty}\left(1-e^{i \beta s}\right) \eta^{\bar{Y}}(s) d s\right) \\
& \rightarrow \exp \left(i \beta|A|+\frac{|A|}{2} \int_{0}^{\infty}\left(1-e^{i \beta s}\right) \eta^{\bar{Y}}(s) d s\right)
\end{aligned}
$$

as $\varepsilon \rightarrow 0$. We note that this last formula is the characteristic function for $\mu_{\star}^{X}(A)$. The calculation for $\mu^{\bar{Y}, \varepsilon}(A)$ uses Lemma 5.4 and a change of variables as follows

$$
\begin{aligned}
\boldsymbol{E}\left[e^{i \beta \mu^{\bar{Y}, \varepsilon}(A)}\right] & =\exp \left(i \beta|A|+\mathbf{1}_{\left[y_{0}, y_{1}\right]}(0) \int_{\frac{2}{\varepsilon} \ell_{0}}^{\frac{2}{\varepsilon} \ell_{1}} \int_{0}^{\infty}\left(1-e^{i \beta s}\right) \eta^{\bar{X}, \varepsilon}(s) d s\right) \\
& =\exp \left(i \beta|A|+\mathbf{1}_{\left[y_{0}, y_{1}\right]}(0) \frac{2\left(\ell_{1}-\ell_{0}\right)}{\varepsilon^{4}} \int_{0}^{\infty}\left(1-e^{i \varepsilon^{2} \beta s}\right) \eta^{\bar{X}, 1}\left(\varepsilon^{-2} s\right) d s\right)
\end{aligned}
$$




$$
=\exp \left(i \beta|A|+\mathbf{1}_{\left[y_{0}, y_{1}\right]}(0) \frac{2\left(\ell_{1}-\ell_{0}\right)}{\varepsilon^{2}} \int_{0}^{\infty}\left(1-e^{i \varepsilon^{2} \beta s}\right) \eta^{\bar{X}, 1}(s) d s\right) .
$$

Notice that by switching the integrals, we find

$$
\begin{aligned}
\frac{1}{\varepsilon^{2}} \int_{0}^{\infty}\left(1-e^{i \beta \varepsilon^{2} s}\right) \eta^{\bar{X}, 1}(s) d s & =\frac{1}{\varepsilon^{2}} \int_{0}^{\infty}\left(-\beta i \varepsilon^{2} \int_{0}^{s} e^{i \beta \varepsilon^{2} r} d r\right) \eta^{\bar{X}, 1}(s) d s \\
& =\int_{0}^{\infty} e^{i \beta \varepsilon^{2} r} \int_{r}^{\infty} \eta^{\bar{X}, 1}(s) d s d r .
\end{aligned}
$$

Since $\eta^{\bar{X}, 1}$ has exponential tails, we can send $\varepsilon \rightarrow 0$, use dominated convergence and switch the integrals again to find

$$
\lim _{\varepsilon \rightarrow 0} \frac{1}{\varepsilon^{2}} \int_{0}^{\infty}\left(1-e^{i \beta \varepsilon^{2} s}\right) \eta^{\bar{X}, 1}(s) d s=\int_{0}^{\infty} s \eta^{\bar{X}, 1}(s) d s=1
$$

and hence

$$
\boldsymbol{E}\left[e^{i \beta \mu^{\bar{Y}, \varepsilon}(A)}\right] \rightarrow \boldsymbol{E}\left[e^{i \beta \mu_{*}^{Y}(A)}\right] .
$$

5.4. Proof of the Excursion Decomposition (Proposition 5.1). To abbreviate the notation, we will now write $L_{t}^{\bar{X}}$ and $L_{t}^{\bar{Y}}$ for $L_{t}^{\bar{X}}(\varepsilon \mathbb{Z})$ and $L_{t}^{\bar{Y}}(0)$, respectively. Notice that $L_{t}^{\bar{X}}$ depends on $\varepsilon$ while $L_{t}^{\bar{Y}}$ does not. Let $X^{\varepsilon}(t)=\bar{X}\left(\psi^{\bar{X}, \varepsilon}(t)\right)$ and $Y^{\varepsilon}(t)=\bar{Y}\left(\psi^{\bar{Y}}, \varepsilon(t)\right)$. The proof of Proposition 5.1 follows quickly from Itô's formula, and the following two lemmas:

Lemma 5.9. For every $t \geqslant 0$, we have

$$
L_{t}^{X^{\varepsilon}}=\frac{2}{\alpha \varepsilon} L_{t}^{Y^{\varepsilon}}
$$

Lemma 5.10. The joint quadratic variation of $X^{\varepsilon}$ and $Y^{\varepsilon}$ is 0 .

Momentarily postponing the proof of these lemmas, we prove Proposition 5.1.

Proof of Proposition 5.1. For any $f \in \mathcal{D}\left(\mathcal{L}^{\varepsilon}\right)$, Itô's formula gives

$$
\begin{aligned}
& \boldsymbol{E} f(\left.Z_{t}^{\varepsilon}\right)-f\left(Z_{0}^{\varepsilon}\right)=\frac{1}{2} \boldsymbol{E} \int_{0}^{\psi^{\bar{X}, \varepsilon}(t)} \partial_{x}^{2} f\left(\bar{X}_{s}, \bar{Y}_{s}\right) \mathbf{1}_{\bar{X}_{s} \notin \varepsilon \mathbb{Z}} d s \\
& \quad+\frac{1}{2} \boldsymbol{E} \int_{0}^{t}\left(\partial_{x} f\left(\left(X_{s}^{\varepsilon}\right)^{+}, Y_{s}^{\varepsilon}\right)-\partial_{x} f\left(\left(X_{s}^{\varepsilon}\right)^{-}, Y_{s}^{\varepsilon}\right)\right) d L_{s}^{X^{\varepsilon}}(\varepsilon \mathbb{Z}) \\
& \quad+\frac{1}{2} \boldsymbol{E} \int_{0}^{\psi^{\bar{Y}, \varepsilon}(t)} \partial_{y}^{2} f\left(\bar{X}_{s}, \bar{Y}_{s}\right) \mathbf{1}_{\bar{Y}_{s} \in(0,1)} d s+\boldsymbol{E} \int_{0}^{t} \partial_{y} f\left(X_{s}^{\varepsilon},\left(Y_{s}^{\varepsilon}\right)^{+}\right) d L_{s}^{Y^{\varepsilon}}(0) .
\end{aligned}
$$

Here we used the fact that $\left\langle X^{\varepsilon}, Y^{\varepsilon}\right\rangle=0$ (Lemma 5.10) and $\partial_{y} f(x, 1)=0$ (which is guaranteed by the assumption $f \in \mathcal{D}\left(\mathcal{L}^{\varepsilon}\right)$ ). Using (5.11) this simplifies to

$$
\begin{gathered}
\boldsymbol{E} f\left(Z_{t}^{\varepsilon}\right)-f\left(Z_{0}^{\varepsilon}\right)=\boldsymbol{E} \int_{0}^{\psi^{\bar{X}, \varepsilon}(t)} \partial_{x}^{2} f\left(\bar{X}_{s}, \bar{Y}_{s}\right) \mathbf{1}_{\bar{X}_{s} \notin \varepsilon \mathbb{Z}} d s \\
+\boldsymbol{E} \int_{0}^{\psi^{\bar{Y}, \varepsilon}(t)} \partial_{y}^{2} f\left(\bar{X}_{s}, \bar{Y}_{s}\right) \mathbf{1}_{\bar{Y}_{s} \in(0,1)} d s \\
+\frac{1}{2} \boldsymbol{E} \int_{0}^{t}\left(\partial_{x} f\left(\left(X_{s}^{\varepsilon}\right)^{+}, Y_{s}^{\varepsilon}\right)-\partial_{x} f\left(\left(X_{s}^{\varepsilon}\right)^{-}, Y_{s}^{\varepsilon}\right)+\alpha \varepsilon \partial_{y} f\left(X_{s}^{\varepsilon},\left(Y_{s}^{\varepsilon}\right)^{+}\right)\right) d L_{s}^{X^{\varepsilon}}(\varepsilon \mathbb{Z}) .
\end{gathered}
$$


Since $f \in \mathcal{D}\left(\mathcal{L}^{\varepsilon}\right)$ and $L^{X^{\varepsilon}}$ only increases when $Y^{\varepsilon}=0$ and $X^{\varepsilon} \in \varepsilon \mathbb{Z}$, the last integral above vanishes. Consequently,

$$
\lim _{t \rightarrow 0} \frac{1}{t} \boldsymbol{E}\left(f\left(Z_{t}^{\varepsilon}\right)-f\left(Z_{0}^{\varepsilon}\right)\right)=\mathcal{L}^{\varepsilon} f(0,0)
$$

showing that the generator of $Z^{\varepsilon}$ is $\mathcal{L}^{\varepsilon}$ as claimed. The fact that $Z^{\varepsilon}$ satisfies (4.4a) and (4.4b) follows immediately by choosing $f(x, y)=x$ and $f(x, y)=y$ respectively.

It remains to prove Lemmas 5.9 and 5.10 .

Proof of Lemma 5.9. We first claim that for any $t \geqslant 0$, we have

$$
\psi^{\bar{X}, \varepsilon}(t)+\psi^{\bar{Y}, \varepsilon}(t)=t .
$$

To see this, define the non-decreasing, right continuous function

$$
H(t) \stackrel{\text { def }}{=} \tau^{\bar{Y}}\left(\frac{\alpha \varepsilon}{2} L_{t}^{\bar{X}}(\varepsilon \mathbb{Z})\right) .
$$

Using the properties of $\tau^{\bar{Y}}, L^{\bar{X}}, \tau^{\bar{X}, \varepsilon}$, and $L^{\bar{Y}}$, it is easy to check that the right continuous inverse of $H$ is

$$
H^{-1}(t)=\inf \{s>0 \mid H(s)>t\}=\tau^{\bar{X}, \varepsilon}\left(\frac{2}{\alpha \varepsilon} L_{s}^{\bar{Y}}(0)\right) .
$$

Therefore, $\psi^{\bar{X}, \varepsilon}$ and $\psi^{\bar{Y}, \varepsilon}$ are the right continuous inverse functions of $t \mapsto t+H(t)$ and $t \mapsto t+H^{-1}(t)$, respectively, meaning that

$$
\begin{aligned}
& \psi^{\bar{X}, \varepsilon}(t)=\inf \{s \mid s+H(s)>t\}, \\
& \psi^{\bar{Y}, \varepsilon}(t)=\inf \left\{r \mid r+H^{-1}(r)>t\right\} .
\end{aligned}
$$

In general, $H\left(H^{-1}(r)\right) \geqslant r$ and $H^{-1}(H(s)) \geqslant s$ must hold, but equality may not hold due to possible discontinuities in $H$ and $H^{-1}$.

Fix $t>0$, and let $\left[t_{0}, t_{1}\right]$ be the maximal interval such that $t \in\left[t_{0}, t_{1}\right]$ and $\psi^{\bar{X}, \varepsilon}$ is constant on the interval $\left[t_{0}, t_{1}\right]$. Possibly $t_{0}=t_{1}=t$, but let us first suppose that the interval has non-empty interior, $t_{0}<t_{1}$. This implies that $H(s)$ has a jump discontinuity at a point $s=\psi^{\bar{X}, \varepsilon}\left(t_{1}\right)$ such that $s+H\left(s^{-}\right)=t_{0}$ and $s+H\left(s^{+}\right)=s+H(s)=t_{1}$. Also, $H^{-1}(H(s))=s$ must hold for such a value of $s$. So, for $\ell=H(s)=H\left(\psi^{\bar{X}, \varepsilon}\left(t_{1}\right)\right)$ we have

$$
\ell+H^{-1}(\ell)=H(s)+s=t_{1} .
$$

Therefore, $\psi^{\bar{Y}, \varepsilon}\left(t_{1}\right)=\ell$, since

$$
\psi^{\bar{Y}, \varepsilon}\left(t_{1}\right)=\inf \left\{r \mid r+H^{-1}(r)>t_{1}\right\} .
$$

This means that $\psi^{\bar{Y}, \varepsilon}\left(t_{1}\right)=H(s)$. Therefore,

$$
\psi^{\bar{Y}, \varepsilon}\left(t_{1}\right)+\psi^{\bar{X}, \varepsilon}\left(t_{1}\right)=H(s)+s=t_{1}
$$

must hold. Now let extend the equality to the rest of the interval $\left[t_{0}, t_{1}\right]$. By assumption, $\psi^{\bar{X}, \varepsilon}(t)=\psi^{\bar{X}, \varepsilon}\left(t_{1}\right)$ for all $t \in\left[t_{0}, t_{1}\right]$. Since $H$ has a jump discontinuity at $s$, this means $H^{-1}(r)$ is constant on the interval $\left[H\left(s^{-}\right), H(s)\right]$. Hence, the function $r+H^{-1}(r)$ is affine with slope 1 on the interval $\left[H\left(s^{-}\right), H(s)\right]=\left[\psi^{\bar{Y}, \varepsilon}\left(t_{1}\right)-\right.$ $\left.\left(t_{1}-t_{0}\right), \psi^{\bar{Y}, \varepsilon}\left(t_{1}\right)\right]$. Therefore, for all $t \in\left[t_{0}, t_{1}\right]$, we must have

$$
\psi^{\bar{Y}, \varepsilon}(t)=\psi^{\bar{Y}, \varepsilon}\left(t_{1}\right)+t-t_{1} .
$$


This shows that for all $t \in\left[t_{0}, t_{1}\right]$, we have

$$
\psi^{\bar{X}, \varepsilon}(t)+\psi^{\bar{Y}, \varepsilon}(t)=\psi^{\bar{X}, \varepsilon}\left(t_{1}\right)+\psi^{\bar{Y}, \varepsilon}\left(t_{1}\right)+t-t_{1}=t .
$$

Applying the same argument with the roles of $\psi^{\bar{X}, \varepsilon}, \psi^{\bar{Y}, \varepsilon}, H$ and $H^{-1}$ reversed, we conclude that $\psi^{\bar{X}, \varepsilon}(t)+\psi^{\bar{Y}, \varepsilon}(t)=t$ must hold if either $\psi^{\bar{X}, \varepsilon}$ or $\psi^{\bar{Y}, \varepsilon}$ is constant on an interval containing $t$ which has non-empty interior. The only other possibility is that both $\psi^{\bar{X}, \varepsilon}$ and $\psi^{\bar{Y}, \varepsilon}$ are strictly increasing through $t$. In this case, $H$ must be continuous at $\psi^{\bar{X}, \varepsilon}(t)$ and $H^{-1}$ must be continuous at $\psi^{\bar{Y}, \varepsilon}(t)$. Thus, $H^{-1}\left(H\left(\psi^{\bar{X}, \varepsilon}(t)\right)\right)=\psi^{\bar{X}, \varepsilon}(t)$ and $H\left(H^{-1}\left(\psi^{\bar{Y}, \varepsilon}(t)\right)\right)=\psi^{\bar{Y}, \varepsilon}(t)$ holds. The rest of the argument is the same as in the previous case. This proves (5.12).

Now, since $X^{\varepsilon}$ and $Y^{\varepsilon}$ are time changes of $\bar{X}$ and $\bar{Y}$ respectively, we know that the local times are given by

$$
L_{t}^{X^{\varepsilon}} \stackrel{\text { def }}{=} L^{X^{\varepsilon}}(\varepsilon \mathbb{Z})=L_{\psi^{\bar{X}, \varepsilon}(t)}^{\overline{X_{1}}}, \quad \text { and } \quad L_{t}^{Y^{\varepsilon} \stackrel{\text { def }}{=}} L^{Y^{\varepsilon}}(0)=L_{\psi^{\bar{Y}, \varepsilon}(t)}^{\overline{\bar{X}}} .
$$

By definition of $\psi^{\bar{X}, \varepsilon}$, we know

$$
t=\psi^{\bar{X}, \varepsilon}(t)+\tau^{\bar{Y}}\left(\frac{\alpha \varepsilon}{2} L^{\bar{X}}\left(\psi^{\bar{X}, \varepsilon}(t)\right)\right) .
$$

Using (5.12) this gives

$$
\psi^{\bar{Y}, \varepsilon}(t)=\tau^{\bar{Y}}\left(\frac{\alpha \varepsilon}{2} L^{\bar{X}}\left(\psi^{\bar{X}, \varepsilon}(t)\right)\right)
$$

and using the fact that $\tau^{\bar{Y}}$ is the inverse of $L^{\bar{Y}}$, we get (5.11) as desired.

Proof of Lemma 5.10. Fix $\delta>0$, and define a sequence of stopping times $0=\sigma_{0}<$ $\theta_{1}<\sigma_{1}<\theta_{2}<\sigma_{2}<\ldots$ inductively, by

$$
\begin{aligned}
\sigma_{0} & =0 \\
\theta_{k+1} & =\inf \left\{t>\sigma_{k} \mid \text { either } Y_{t}^{\varepsilon}=\delta \text { or } d\left(X_{t}^{\varepsilon}, \varepsilon \mathbb{Z}\right)=\delta\right\}, \quad k=0,1,2,3, \ldots \\
\sigma_{k+1} & =\inf \left\{t>\theta_{k} \mid Y_{t}=0 \text { and } X_{t}^{\varepsilon} \in \varepsilon \mathbb{Z}\right\}, \quad k=0,1,2,3, \ldots
\end{aligned}
$$

Then for $T>0$, we decompose the joint quadratic variation over $[0, T]$ as

$$
\left\langle X^{\varepsilon}, Y^{\varepsilon}\right\rangle_{[0, T]}=\sum_{k \geqslant 0}\left\langle X^{\varepsilon}, Y^{\varepsilon}\right\rangle_{\left[\sigma_{k} \wedge T, \theta_{k+1} \wedge T\right]}+\left\langle X^{\varepsilon}, Y^{\varepsilon}\right\rangle_{\left[\theta_{k+1} \wedge T, \sigma_{k+1} \wedge T\right]} .
$$

We claim that for all $k$,

$$
\left\langle X^{\varepsilon}, Y^{\varepsilon}\right\rangle_{\left[\theta_{k+1} \wedge T, \sigma_{k+1} \wedge T\right]}=0
$$

holds with probability one. Hence,

$$
\begin{aligned}
\left|\left\langle X^{\varepsilon}, Y^{\varepsilon}\right\rangle_{[0, T]}\right| & \leqslant \sum_{k \geqslant 0}\left|\left\langle X^{\varepsilon}, Y^{\varepsilon}\right\rangle_{\left[\sigma_{k} \wedge T, \theta_{k+1} \wedge T\right]}\right| \\
& \leqslant \sum_{k \geqslant 0} \frac{1}{2}\left\langle X^{\varepsilon}, X^{\varepsilon}\right\rangle_{\left[\sigma_{k} \wedge T, \theta_{k+1} \wedge T\right]}+\frac{1}{2}\left\langle Y^{\varepsilon}, Y^{\varepsilon}\right\rangle_{\left[\sigma_{k} \wedge T, \theta_{k+1} \wedge T\right]} \\
& \leqslant \sum_{k \geqslant 0}\left|\left(\theta_{k+1} \wedge T\right)-\left(\sigma_{k} \wedge T\right)\right| \\
& \leqslant \mid\left\{t \in[0, T]|\quad| \bar{Y}_{t} \mid \leqslant \delta, \text { and } d\left(\bar{X}_{t}, \varepsilon \mathbb{Z}\right) \leqslant \delta \quad\right\} \mid .
\end{aligned}
$$

As $\delta \rightarrow 0$, the latter converges to 0 almost surely, which proves that $\left\langle X^{\varepsilon}, Y^{\varepsilon}\right\rangle=0$.

To establish the claim (5.13), we may assume $\theta_{k}<T$, for otherwise, the statement is trivial. At time $\theta_{k}$, we have either $X_{\theta_{k}}^{\varepsilon} \notin \varepsilon \mathbb{Z}$ or $Y_{\theta_{k}}=\delta$. In the former case, 
we must have $X_{t} \notin \varepsilon \mathbb{Z}$ for all $t \in\left[\theta_{k}, \sigma_{k}\right)$. Hence, $\psi^{\bar{Y}, \varepsilon}(t)$ and $Y_{t}^{\varepsilon}$ are constant for all $t \in\left[\theta_{k}, \sigma_{k}\right)$. In the other case, $Y_{t}>0$ for all $t \in\left[\theta_{k}, \sigma_{k}\right)$ while $X_{t}$ is constant on $\left[\theta_{k}, \sigma_{k}\right]$. In either case, this implies that $\left\langle X^{\varepsilon}, Y^{\varepsilon}\right\rangle_{\left[\theta_{k} \wedge T, \sigma_{k} \wedge T\right]}=0$ holds with probability one.

\section{References}

[ADH90] T. Arbogast, J. Douglas, Jr., and U. Hornung. Derivation of the double porosity model of single phase flow via homogenization theory. SIAM J. Math. Anal., 21(4):823-836, 1990. doi:10.1137/0521046.

[BAC +15$]$ G. Ben Arous, M. Cabezas, J. Černý, and R. Royfman. Randomly trapped random walks. Ann. Probab., 43(5):2405-2457, 09 2015. doi:10.1214/14-AOP939.

[BAČ07] G. Ben Arous and J. Černý. Scaling limit for trap models on $\mathbb{Z}^{d}$. Ann. Probab., 35(6):2356-2384, 2007. doi:10.1214/009117907000000024.

[Bas87] A. B. Basset. On the motion of two spheres in a liquid, and allied problems. Proc. Lond. Math. Soc., 18:369-377, 1886/87. doi:10.1112/plms/s1-18.1.369.

[BDB14] A. M. Berezhkovskii, L. Dagdug, and S. M. Bezrukov. From normal to anomalous diffusion in comb-like structures in three dimensions. The Journal of Chemical Physics, 141(5):054907, 2014. doi:10.1063/1.4891566.

[BE07] P. C. Bressloff and B. A. Earnshaw. Diffusion-trapping model of receptor trafficking in dendrites. Phys. Rev. E, 75:041915, Apr 2007. doi:10.1103/PhysRevE.75.041915.

[Ber06] D. Bertacchi. Asymptotic behaviour of the simple random walk on the 2-dimensional comb. Electron. J. Probab., 11:no. 45, 1184-1203, 2006. doi:10.1214/EJP.v11-377.

[BLM96] A. Bourgeat, S. Luckhaus, and A. Mikelić. Convergence of the homogenization process for a double-porosity model of immiscible two-phase flow. SIAM J. Math. Anal., 27(6):1520-1543, 1996. doi:10.1137/S0036141094276457.

[BMN09] B. Baeumer, M. M. Meerschaert, and E. Nane. Brownian subordinators and fractional Cauchy problems. Trans. Amer. Math. Soc., 361(7):3915-3930, 2009. doi:10.1090/S0002-9947-09-04678-9.

[BZ03] D. Bertacchi and F. Zucca. Uniform asymptotic estimates of transition probabilities on combs. J. Aust. Math. Soc., 75(3):325-353, 2003. doi:10.1017/S1446788700008144.

[CCFR09] E. Csáki, M. Csörgö, A. Földes, and P. Révész. Strong limit theorems for a simple random walk on the 2-dimensional comb. Electron. J. Probab., 14:no. 82, 2371-2390, 2009. doi:10.1214/EJP.v14-710.

[CCFR11] E. Csáki, M. Csörgö, A. Földes, and P. Révész. On the local time of random walk on the 2-dimensional comb. Stochastic Process. Appl., 121(6):1290-1314, 2011. doi:10.1016/j.spa.2011.01.009.

[Che17] Z.-Q. Chen. Time fractional equations and probabilistic representation. Chaos Solitons Fractals, 102:168-174, 2017. doi:10.1016/j.chaos.2017.04.029.

[Cla98] G. W. Clark. Derivation of microstructure models of fluid flow by homogenization. J. Math. Anal. Appl., 226(2):364-376, 1998. doi:10.1006/jmaa.1998.6085.

[Coh18] S. Cohn. On the Homogenization of Diffusions in Periodic Comb-Like Structures. ProQuest LLC, Ann Arbor, MI, 2018. Thesis (Ph.D.)-Carnegie Mellon University.

[DBMZ07] L. Dagdug, A. M. Berezhkovskii, Y. A. Makhnovskii, and V. Y. Zitserman. Transient diffusion in a tube with dead ends. The Journal of Chemical Physics, 127(22):224712, 2007. doi:10.1063/1.2805068.

[Die10] K. Diethelm. The analysis of fractional differential equations, volume 2004 of Lecture Notes in Mathematics. Springer-Verlag, Berlin, 2010. doi:10.1007/978-3-642-14574-2.

[Dro00] J. Droniou. Solving convection-diffusion equations with mixed, Neumann and Fourier boundary conditions and measures as data, by a duality method. Adv. Differential Equations, 5(10-12):1341-1396, 2000.

[EK86] S. N. Ethier and T. G. Kurtz. Markov processes: characterization and convergence. Wiley Series in Probability and Mathematical Statistics: Probability and Mathematical Statistics. John Wiley \& Sons, Inc., New York, 1986. doi:10.1002/9780470316658.

[EP14] H.-J. Engelbert and G. Peskir. Stochastic differential equations for sticky Brownian motion. Stochastics, 86(6):993-1021, 2014. doi:10.1080/17442508.2014.899600.

[Fel52] W. Feller. The parabolic differential equations and the associated semi-groups of transformations. Ann. of Math. (2), 55:468-519, 1952. doi:10.2307/1969644. 
[Fel54] W. Feller. Diffusion processes in one dimension. Trans. Amer. Math. Soc., 77:1-31, 1954. doi:10.2307/1990677.

[Fri75] A. Friedman. Stochastic differential equations and applications. Vol. 1. Academic Press [Harcourt Brace Jovanovich, Publishers], New York-London, 1975. Probability and Mathematical Statistics, Vol. 28.

[FS00] M. Freidlin and S.-J. Sheu. Diffusion processes on graphs: stochastic differential equations, large deviation principle. Probab. Theory Related Fields, 116(2):181-220, 2000. doi:10.1007/PL00008726.

[FW93] M. I. Freidlin and A. D. Wentzell. Diffusion processes on graphs and the averaging principle. Ann. Probab., 21(4):2215-2245, 1993. doi:10.1214/aop/1176989018.

[FW12] M. I. Freidlin and A. D. Wentzell. Random perturbations of dynamical systems, volume 260 of Grundlehren der Mathematischen Wissenschaften [Fundamental Principles of Mathematical Sciences]. Springer, Heidelberg, third edition, 2012. doi:10.1007/978-3-642-25847-3. Translated from the 1979 Russian original by Joseph Szücs.

[Gri85] P. Grisvard. Elliptic problems in nonsmooth domains, volume 24 of Monographs and Studies in Mathematics. Pitman (Advanced Publishing Program), Boston, MA, 1985.

$\left[\mathrm{HIK}^{+} 18\right]$ M. Hairer, G. Iyer, L. Koralov, A. Novikov, and Z. Pajor-Gyulai. A fractional kinetic process describing the intermediate time behaviour of cellular flows. Ann. Probab., 46(2):897-955, 2018. doi:10.1214/17-AOP1196.

[HKPG16] M. Hairer, L. Koralov, and Z. Pajor-Gyulai. From averaging to homogenization in cellular flows - an exact description of the transition. Ann. Inst. Henri Poincaré Probab. Stat., 52(4):1592-1613, 2016. doi:10.1214/15-AIHP690.

[How07] C. J. Howitt. Stochastic flows and sticky brownian motion. August 2007. URL http://wrap.warwick.ac.uk/56226/.

[HS14] D. Holcman and Z. Schuss. The narrow escape problem. SIAM Rev., 56(2):213-257, 2014. doi:10.1137/120898395.

[IM74] K. Itô and H. P. McKean, Jr. Diffusion processes and their sample paths. SpringerVerlag, Berlin-New York, 1974. Second printing, corrected, Die Grundlehren der mathematischen Wissenschaften, Band 125.

[Itô72] K. Itô. Poisson point processes attached to Markov processes. In Proceedings of the Sixth Berkeley Symposium on Mathematical Statistics and Probability (Univ. California, Berkeley, Calif., 1970/1971), Vol. III: Probability theory, pages 225-239. Univ. California Press, Berkeley, Calif., 1972.

[KS91] I. Karatzas and S. E. Shreve. Brownian motion and stochastic calculus, volume 113 of Graduate Texts in Mathematics. Springer-Verlag, New York, second edition, 1991. doi:10.1007/978-1-4612-0949-2.

[Kyp06] A. E. Kyprianou. Introductory lectures on fluctuations of Lévy processes with applications. Universitext. Springer-Verlag, Berlin, 2006.

[MS15] M. Magdziarz and R. L. Schilling. Asymptotic properties of Brownian motion delayed by inverse subordinators. Proc. Amer. Math. Soc., 143(10):4485-4501, 2015. doi: $10.1090 / \mathrm{proc} / 12588$.

[PY07] J. Pitman and M. Yor. Itô's excursion theory and its applications. Jpn. J. Math., 2(1):83-96, 2007. doi:10.1007/s11537-007-0661-z.

[RW00] L. C. G. Rogers and D. Williams. Diffusions, Markov processes, and martingales. Vol. 2. Cambridge Mathematical Library. Cambridge University Press, Cambridge, 2000. doi:10.1017/CBO9781107590120. Itô calculus, Reprint of the second (1994) edition.

[SV71] D. W. Stroock and S. R. S. Varadhan. Diffusion processes with boundary conditions. Comm. Pure Appl. Math., 24:147-225, 1971. doi:10.1002/cpa.3160240206.

[SW91] R. E. Showalter and N. J. Walkington. Diffusion of fluid in a fissured medium with microstructure. SIAM J. Math. Anal., 22(6):1702-1722, 1991. doi:10.1137/0522105.

[Uma12] S. Umarov. On fractional Duhamel's principle and its applications. J. Differential Equations, 252(10):5217-5234, 2012. doi:10.1016/j.jde.2012.01.029.

[US06] S. Umarov and E. Saydamatov. A fractional analog of the Duhamel principle. Fract. Calc. Appl. Anal., 9(1):57-70, 2006.

[You88] W. R. Young. Arrested shear dispersion and other models of anomalous diffusion. J. Fluid Mech., 193:129-149, Aug 1988. doi:10.1017/S0022112088002083. 